\title{
Supplementary Information for Controls on Erosion in the Western Tarim Basin: Implications for the Uplift of Northwest Tibet and Pamirs
}

Major and Trace Element Geochemistry

Each bulk, unsieved sediment sample was analyzed for major and trace elements to provide a basic characterization of the material that was also assessed with other isotopic and thermochronologic methods. Carbonate was not removed prior to digestion. For elemental analysis all samples were freeze-dried and ground before mixing $600 \mathrm{mg}$ of sample with $3600 \mathrm{mg}$ of lithium tetraborate $\left(\mathrm{Li}_{2} \mathrm{~B}_{4} \mathrm{O}_{7}\right.$, Spektromelt). The samples were pre-oxidized at $500^{\circ} \mathrm{C}$ with $\mathrm{NH}_{4} \mathrm{NO}_{3}$ and fused to glass-beads. Samples were then were analyzed for $\mathrm{Si}, \mathrm{Al}, \mathrm{Ti}, \mathrm{Fe}, \mathrm{Na}, \mathrm{Ca}, \mathrm{K}$, P and Rb by X-Ray Fluorescence (XRF) using a Philips PW 2400 X-Ray spectrometer at the Institut für Chemie und Biologie des Meeres (ICBM) at the Carl von Ossietzky Universität, Oldenburg, Germany. XRF measurements were performed using the method of Böning et al. (2009). Overall analytical precision and accuracy were monitored by measurements of several inhouse standards and the certified standard GSD-12, and were better than 3\%. All data are presented in Table 2.

Isotope Geochemistry

Sediments were also analyzed for $\mathrm{Sr}$ and $\mathrm{Nd}$ isotopes because these systems have an established track record of being reliable provenance and chemical weathering proxies in sedimentary systems. $\mathrm{Nd}$ is a water-immobile element and is generally considered not to experience isotopic fractionation during weathering and erosion processes (Goldstein et al., 1984). The Nd isotopic composition broadly reflects the average age and lithology of the crust being 
eroded so that sediments from ancient continental crust have different signatures compared to those derived from younger igneous bodies. Sr is water mobile and may be affected by weathering processes, as well as the provenance (Derry and France-Lanord, 1996). Unweathered rocks show correlation between $\mathrm{Nd}$ and $\mathrm{Sr}$ isotopes, but ${ }^{87} \mathrm{Sr} /{ }^{86} \mathrm{Sr}$ values tend to increase with stronger alteration (Derry and France-Lanord, 1996). Sr isotopes are strongly affected by the presence of carbonate and care was taken to decarbonate samples prior to analysis. This was especially important in the Tarim Basin because of the presence of common carbonate grains in the sediment. This means that our isotopic analysis only constrains the provenance of the siliciclastic fraction.

Prior to total digestion all samples were leached using buffered acetic acid to remove any carbonate-bound Sr. This was followed by a leach with $25 \%(\mathrm{v} / \mathrm{v})$ acetic acid and $0.02 \mathrm{M}$ hydroxylamine hydrochloride $(\mathrm{HH})$ to remove $\mathrm{Sr}$ contained in authigenic $\mathrm{Mn}$-Fe-oxides, which may also concentrate $\mathrm{Nd}$. Hence, the $\mathrm{Sr}$ and $\mathrm{Nd}$ isotopic signatures is assumed to result solely from the silicate fraction (plus perhaps fractions of dolomite). The leached sediments were then digested in closed PTFE vessels (Böning et al., 2004). The samples and the certified standard BCR-2 $(50 \mathrm{mg})$, as well as a blank, were treated with $\mathrm{HNO}_{3}$ overnight to oxidize any organic matter. After that $\mathrm{HF}$ and $\mathrm{HClO}_{4}$ were added and the vessels were heated for $12 \mathrm{~h}$ at $180^{\circ} \mathrm{C}$. All acids were of ultra-pure quality. After digestion, acids were evaporated on a heated metal block $\left(180^{\circ} \mathrm{C}\right)$, residues were redissolved and fumed off three times with $6 \mathrm{~N} \mathrm{HCl}$, and finally taken up in $1 \mathrm{~N}^{-N_{3}}$.

From the resulting solutions, rare earth elements (REEs) and $\mathrm{Sr}$ were isolated from major elements and separated from each other by two-step column chemistry using Eichrom TRU-Spec resin. Nd was then isolated from interfering REEs using Eichrom LN-Spec resin with 0.23-0.25 
$\mathrm{N} \mathrm{HCl}$ as eluant. The TRU-Spec Sr-Rb cut was loaded on Eichrom Sr-Spec columns to isolate $\mathrm{Sr}$ using Milli-Q water. The isotopic compositions of $\mathrm{Nd}$ and $\mathrm{Sr}$ were analyzed on a Thermo Neptune Plus Multicollector ICP-MS at the ICBM, Oldenburg. For Nd isotope analyses, all samples were corrected for internal mass fractionation using ${ }^{146} \mathrm{Nd} /{ }^{144} \mathrm{Nd}=0.7219$ and an exponential law. Each measurement session was accompanied by multiple analyses of the $\mathrm{Nd}$ standard JNdi-1 with sample-like concentrations, and ${ }^{143} \mathrm{Nd} /{ }^{144} \mathrm{Nd}$ ratios of all samples were normalized to the reported JNdi-1 value of ${ }^{143} \mathrm{Nd} /{ }^{144} \mathrm{Nd}=0.512115$ (Tanaka et al., 2000). The $\mathrm{Nd}$ isotopic composition is expressed in $\varepsilon_{\mathrm{Nd}}$ notation:

$$
\varepsilon_{\mathrm{Nd}}=\left[\left({ }^{143} \mathrm{Nd} /{ }^{144} \mathrm{Nd}\right)_{\text {sample }} /\left({ }^{143} \mathrm{Nd} /{ }^{144} \mathrm{Nd}\right)_{\mathrm{CHUR}}-1\right] * 10^{4}
$$

$\left({ }^{143} \mathrm{Nd} /{ }^{144} \mathrm{Nd}\right)_{\text {CHUR }}$ is the Chondritic Uniform Reservoir with a value of 0.512638 (Jacobsen and Wasserburg, 1980). The external reproducibility is calculated for each session separately using the analyses of JNdi-1 and was generally better than \pm 0.000015 or $\pm 0.3 \varepsilon_{\mathrm{Nd}}$ units $(2 \sigma)$. The BCR2 standard $(\mathrm{n}=4)$ had an $\varepsilon_{\mathrm{Nd}}$ value of $0.1( \pm 0.3,2 \sigma)$ and was well within the reported $\varepsilon_{\mathrm{Nd}}$ value of $0.0 \pm 0.2$ (Raczek et al., 2003). The procedural blank was $\leq 30 \mathrm{pg} \mathrm{Nd}$.

For Sr isotope analyses, all samples were corrected for mass fractionation using ${ }^{86} \mathrm{Sr}^{/ 88} \mathrm{Sr}$ $=0.1194$ and the exponential law. Measurements were accompanied by multiple analyses of NBS987 with sample-like concentrations, and ${ }^{87} \mathrm{Sr}^{186} \mathrm{Sr}$ ratios of all samples were normalized to the reported value of 0.710248 (Thirlwall, 1991). Furthermore, $\mathrm{Kr}$, $\mathrm{Rb}$ and $\mathrm{Ba}$ contents were monitored and found to be negligible. The external reproducibility is calculated using the analyses of NBS987 and was generally better than 50 ppm $(2 \sigma)$. The BCR-2 standard $(n=4)$ had $\mathrm{a}^{87} \mathrm{Sr} /{ }^{86} \mathrm{Sr}$ ratio of $0.70502 \pm 0.00004(2 \sigma)$ and was within the reported ${ }^{87} \mathrm{Sr} /{ }^{86} \mathrm{Sr}$ ratio of 0.70496 \pm 0.00002 (Raczek et al., 2003). The procedural blanks were negligible throughout. Results are reported in Table 2 . 


\section{Detrital zircon U-Pb geochronology}

Detrital zircon $\mathrm{U}-\mathrm{Pb}$ dating has become a popular and effective technique for evaluating sediment provenance in clastic systems because zircon is a common mineral in continental rocks of many compositions, and is chemically and mechanically durable enough to survive multiple cycles of erosion, transport and sedimentation (Gehrels, 2014). Grains $>50 \mu \mathrm{m}$ across were analyzed, reflecting the spot size of the laser employed. Samples were separated for zircon using standard magnetic and heavy liquid separation techniques at Birkbeck, University of London, UK. The $>\# 40$ sieve-fraction was separated using a vibrating Gemini ${ }^{\circledR}$ Mineral Separation table. The densest fraction was collected, washed with ethanol, and dried in an oven at $100^{\circ} \mathrm{C}$. Once dry, a rare-earth element hand magnet was passed several times over the sample to remove extremely magnetic material and sieved again to $63-250 \mu \mathrm{m}$ before passing through a Frantz ${ }^{\circledR}$ Magnetic Barrier Laboratory Separator Model LB-1 to isolate nonmagnetic (>1.6 A) grains. Prior to heavy liquid separation, all samples were pre-treated using hydrogen peroxide, acetic acid, and oxalic

acid to remove organic material, carbonate, and Fe-oxides, respectively. Extracted detrital zircons were picked and mounted in epoxy resin, polished, and imaged by reflected light and cathodoluminescence.

Detrital zircons were dated using the U-Pb method at the London Geochronology Centre facilities at University College London, using a New Wave Nd:YAG $193 \mathrm{~nm}$ laser ablation system, coupled to an Agilent 7700 quadrupole ICP-MS. Around 100-120 grains are considered generally sufficient for characterizing sand eroded from a geologically complicated drainage basin (Vermeesch, 2004). Real time U-Pb data were processed using GLITTER 4.4 data 
reduction software. Repeated measurements of external zircon standard Plesovice (TIMS reference age 337.13 $\pm 0.37 \mathrm{Ma}$ )(Sláma et al., 2008) and NIST 612 silicate glass (Pearce et al., 1997) were used to correct for instrumental mass bias and depth-dependent inter-element

fractionation of $\mathrm{Pb}$, Th and $\mathrm{U}$. For this study ${ }^{206} \mathrm{~Pb} /{ }^{238} \mathrm{U}$ ages are used for grains younger than $1000 \mathrm{Ma}$, and for zircon grains older than $1000 \mathrm{Ma}$ we used the ${ }^{207} \mathrm{~Pb} /{ }^{206} \mathrm{~Pb}$ ages to calculate the crystallization age. Because some grains are discordant we chose to only plot those grains when the discordance was less than $15 \%$. Table 4 .

\section{Detrital Apatite Fission Track Geochronology}

Three samples were analyzed for apatite fission track dating. The low-temperature apatite fission-track method, which records cooling through $\sim 60-125^{\circ} \mathrm{C}$ over timescales of $1-10$ m.y. (Green et al., 1989) is particularly sensitive to exhumation driven by erosion and has been widely used in exhumation studies worldwide. Apatites were picked, mounted, and analyzed after heavy mineral extraction at University College, London, UK. Polished grain mounts of apatite were etched with $5 \mathrm{~N} \mathrm{HNO}_{3}$ at $20^{\circ} \mathrm{C}$ for $20 \mathrm{~s}$ to reveal the spontaneous fission-tracks. Etched grain mounts were packed with mica external detectors and corning glass (CN5) dosimeters and irradiated in the FRM 11 thermal neutron facility at the University of Munich in Germany. Following irradiation the external detectors were etched using $48 \% \mathrm{HF}$ at $20^{\circ} \mathrm{C}$ for 25 minutes. Sample ages were determined using the zeta calibration method and IUGS recommended age standards (Hurford, 1990). The results of the fission track analyses are presented in Table 5. 


\section{Optically Stimulated Luminescence Dating}

Depositional ages of sediment in the terraces were determined by optically stimulated luminescence (OSL) dating of quartz sand. While OSL dating can be challenging in fluvial environments, deposits from these settings can be accurately dated by selecting depositional facies most likely to have been reset by sunlight exposure (Fuchs and Owen, 2008; Rittenour, 2008; Wyshnytzky et al., 2015). We preferentially targeted well-sorted, horizontally bedded sand lenses from fluvial deposits to reduce the influence of incomplete resetting (partial bleaching) of the luminescence signal.

Six OSL samples were collected by pounding metal pipes into surface exposures and were shipped to the Utah State University (USU) Luminescence Laboratory for optically stimulated luminescence (OSL) dating of quartz sand (Huntley et al., 1985; Murray and Wintle, 2000). Samples were opened under dim amber light $(\sim 590 \mathrm{~nm})$ and sediment from the inner portions of the sample tubes was sieved and treated with hydrochloric acid to dissolve carbonates and chlorine bleach to remove organic material. Heavy mineral separation (sodium polytungstate, $2.7 \mathrm{~g} / \mathrm{cm}^{3}$ ) and concentrated hydrofluoric and hydrochloric acids were used to remove feldspars, etched the quartz grains, and prevented formation of fluorite precipitates (see Rittenour et al. 2005 for details). The samples were re-sieved to remove the $<63 \mu \mathrm{m}$ grain-size fraction of etched quartz and any partially dissolved feldspars (Wintle, 1997). Purity of the samples was checked using infrared (IR) stimulation on all aliquots.

Representative sediment surrounding the sample tubes was collected for dose rate analysis and submitted for ICP-MS and ICP-AES analyses of $\mathrm{K}, \mathrm{Rb}$, Th, and U content. In-situ gravimetric moisture content was measured for all samples. For those with $<5.8 \%$ measured water content, we used an average value of $5.8 \pm 3.0 \%$ to represent the moisture content over 
burial history. Dose rate calculations include cosmic dose rate contribution by using sample depth, elevation, and longitude/latitude following Prescott and Hutton (1994), influence of water attenuation, uncertainty in elemental measurements and dose rate conversion factors (Aitken, 1998; Guerin et al., 2011).

The OSL age (ka) is determined by dividing the lab-derived radiation dose required to replicate the natural luminescence signal (in greys, Gy), termed equivalent dose $\left(D_{E}\right)$, by the environmental dose rate (in Gy/ka) of the surrounding sediments (Aitken, 1998). Individual aliquot $\mathrm{D}_{\mathrm{E}}$ 's were calculated using the SAR technique of Murray and Wintle (2000) on 2-mm diameter aliquots ( $\sim 35$ grains per disk). Optical measurements were performed on Risø TL/OSL Model DA-20 readers with blue-green light emitting diodes (LED) $(470 \pm 30 \mathrm{~nm})$ as the stimulation source, and reader dose rates of $0.12-0.13 \mathrm{~Gy} / \mathrm{sec}$ from a decaying ${ }^{90} \mathrm{Sr}$ beta source (Bøtter-Jensen et al., 2000). The luminescence signal was measured through 7.5-mm UV filters (U-340) over 40 seconds ( 250 channels) at $125^{\circ} \mathrm{C}$ with LED diodes $\left(\sim 45 \mathrm{~mW} / \mathrm{cm}^{2}\right)$, and calculated by subtracting the average of the last five seconds (background signal) from the first 0.7 seconds (4 channels) of the signal decay curve.

A preheat-plateau (PP) test was performed on USU-1490 to determine the proper preheat temperature(Murray and Wintle, 2003). For the PP test, five 1-mm aliquots were used at each temperature step with preheat temperatures increased by $20^{\circ} \mathrm{C}$ increments from $180^{\circ} \mathrm{C}$ to $260^{\circ} \mathrm{C}$, and held for 10 seconds. First, each non-heated and non-irradiated aliquot was optically bleached at room temperature twice using blue-green LED diodes at $90 \%$ power for 40 seconds, each followed by a 990-second pause to let unstable, thermally-transferred charges decay ( $\mathrm{Li}$ and $\mathrm{Li}$, 2006). Aliquots were then irradiated with $11.8 \mathrm{~Gy}$ of beta radiation. A thermal transfer (TT) test was also conducted on sample USU-1490. This test was similar to the PP test except no dose 
was given following bleaching. Recovered PP and TT doses were measured using the SAR protocol with a $160^{\circ} \mathrm{C}$ preheat following each test doses (each held for 10 seconds), and preheat temperatures ranging from 180 to $260^{\circ} \mathrm{C}$ (held for $10 \mathrm{~s}$ ). Results from these tests were variable with preheat temperature but suggest that preheat temperatures between $200^{\circ} \mathrm{C}$ and $260^{\circ} \mathrm{C}$ are optimal for dose recovery near unity and minimal thermal transfer. A preheat temperature of $200^{\circ} \mathrm{C}$ was used for all samples except USU-1495 where a $260^{\circ} \mathrm{C}$ preheat temperature was used.

Cumulative $\mathrm{D}_{\mathrm{E}}$ values were calculated using the central age model (CAM) and the 3parameter of Galbraith and Roberts (2012)(Fig. S2). Samples with high overdispersion (>30\%) and positive skew of the $\mathrm{D}_{\mathrm{E}}$ data distribution were calculated using the minimum age model. Aliquots were rejected if they had evidence of feldspar contamination, $>20 \%$ recycling ratio or recuperated signal, natural $\mathrm{D}_{\mathrm{E}}$ greater than the highest regenerative dose given, or poor dose response curve fit. Errors on $\mathrm{D}_{\mathrm{E}}$ and age estimates are reported at 2-sigma standard error and were calculated in quadrature using the methods of Aitken and Alldred (1972) and Guerin et al. (2011).

\section{REFERENCES CITED}

Aitken, M. J., 1998, An introduction to optical dating, Oxford, Oxford University Press, 240 p. Aitken, M. J., and Allred, J. C., 1972, The assessment of error limits in thermoluminescence dating: Archaeometry, v. 14, p. 257-267.

Böning, P., Brumsack, H.-J., Schnetger, B., and Grunwald, M., 2009, Trace metal signatures of Chilean upwelling sediments at $\sim 36^{\circ} \mathrm{S}$ : Marine Geology, v. 259, p. 112-121.

Böning, P., Brumsack, H. J., Böttcher, M. E., Schnetger, B., Kriete, C., Kallmeyer, J., and Borchers, S. L., 2004, Geochemistry of Peruvian near-surface sediments: Geochimica et Cosmochimica Acta, v. 68, p. 4429-4451.

Bøtter-Jensen, L., Solongo, S., Murray, A. S., Banerjee, D., and Jungner, H., 2000, Using the OSL single-aliquot regenerative-dose protocol with quartz extracted from building materials in retrospective dosimetry: Radiation Measurements, v. 32, p. 841-845. 
Derry, L. A., and France-Lanord, C., 1996, Neogene Himalayan weathering history and river ${ }^{87} \mathrm{Sr} /{ }^{86} \mathrm{Sr}$; impact on the marine Sr record: Earth and Planetary Science Letters, v. 142, p. 59-74.

Fuchs, M., and Owen, L. A., 2008, Luminescence dating of glacial and associated sediments: review, recommendations and future directions: Boreas, v. 37, p. 636-659.

Galbraith, R. F., and Roberts, R. G., 2012, Statistical aspects of equivalent dose and error calculation and display in OSL dating: An overview and some recommendations: Quaternary Geochronology, v. 11, p. 1-27.

Gehrels, G. E., 2014, Detrital Zircon U-Pb Geochronology Applied to Tectonics: Annual Review of Earth and Planetary Sciences, v. 42, p. 127-149. doi: 10.1146/annurev-earth-050212124012.

Goldstein, S. L., O'Nions, R. K., and Hamilton, P. J., 1984, A Sm-Nd isotopic study of atmospheric dusts and particulates from major river systems: Earth and Planetary Science Letters, v. 70, no. 2, p. 221-236.

Green, P. F., Duddy, I. R., Laslett, G. M., Hegarty, K. A., Gleadow, A. J. W., and Lovering, J. F., 1989, Thermal annealing of fission tracks in apatite; 4, Quantitative modelling techniques and extension to geological timescales: Chemical Geology; Isotope Geoscience Section, v. 79 , no. 2, p. 155-182.

Guerin, G., Mercier, N., and Adamiec, G., 2011, Dose rate conversion factors: update: Ancient Thermo Luminesence, v. 29, no. 1, p. 5-8.

Huntley, D. J., Godfrey-Smith, D. I., and Thewalt, M. L. W., 1985, Optical dating of sediments: Nature, v. 313, p. 105-107.

Hurford, A., 1990, Standardization of fission track dating calibration: Recommendation by the Fission Track Working Group of the IUGS subcommission on geochronology: Chemical Geology, v. 80, p. 177-178.

Jacobsen, S. B., and Wasserburg, G. J., 1980, Sm-Nd isotopic evolution of chondrites: Earth and Planetary Science Letters v. 50, no. 1, p. 139-155.

Li, B., and Li, S.-H., 2006, Studies of thermal stability of charges associated with thermal transfer of OSL from quartz: Journal of Physics D: Applied Physics, v. 39, no. 14. doi:10.1088/0022-3727/39/14/011.

Murray, A. S., and Wintle, A. G., 2000, Luminescence dating of quartz using an improved singlealiquot regenerative-dose protocol: Radiation Measurements, v. 32, p. 57-72.

-, 2003, The single aliquot regenerative dose protocol: potential for improvements in reliability: Radiation measurements, v. 37, no. 4, p. 377-381.

Pearce, N. J. G., Perkins, W. T., Westgate, J. A., Gorton, M. P., Jackson, S. E., Neal, C. R., and Chenery, S. P., 1997, A compilation of new and published major and trace element data for NIST SRM 610 and NIST SRM 612 glass reference materials: Geostandards

Newsletter-the Journal of Geostandards and Geoanalysis, v. 21, no. 1, p. 115-144.

Prescott, J. R., and Hutton, J. T., 1994, Cosmic ray contributions to dose rates for luminescence and ESR dating: large depths and long-term time variations: Radiation Measurements, v. 23, p. 497-500.

Raczek, I., Jochum, K. P., and Hofmann, A. W., 2003, Neodymium and Strontium Isotope Data for USGS Reference Materials BCR-1, BCR-2, BHVO-1, BHVO-2, AGV-1, AGV-2, GSP-1, GSP-2 and Eight MPI-DING Reference Glasses: Geostandards Newsletter, v. 27, no. 2, p. 173-179. doi;10.1111/j.1751-908X.2003.tb00644.x. 
Rittenour, T. M., 2008, Luminescence dating of fluvial deposits: applications to geomorphic, palaeoseismic and archaeological research: Boreas v. 37, p. 613-635.

Sláma, J., Košler, J., Condon, D. J., Crowley, J. L., Gerdes, A., Hanchar, J. M., Horstwood, M. S. A., Morris, G. A., Nasdala, L., Norberg, N., Schaltegger, U., Schoene, B., Tubrett, M. N., and Whitehouse, M. J., 2008, Plezovice zircon A new natural reference material for U-Pb and Hf isotopic microanalysis: Chemical Geology, v. 249, p. 1-35. doi:10.1016/j.chemgeo.2007.11.005.

Tanaka, T., Togashi, S., Kamioka, H., Amakawa, H., Kagami, H., Hamamoto, T., Yuhara, M., Orihashi, Y., Yoneda, S., Shimizu, H., Kunimaru, T., Takahashi, K., Yanagi, T., Nakano, T., Fujimaki, H., Shinjo, R., Asahara, Y., Tanimizu, M., and Dragusanu, C., 2000, JNdi-1: a neodymium isotopic reference in consistency with LaJolla neodymium: Chemical Geology, v. 168, p. 279-281.

Thirlwall, M. F., 1991, Long-term reproducibility of multicollector $\mathrm{Sr}$ and $\mathrm{Nd}$ isotope ratio analysis: Chemical Geology: Isotope Geoscience section, v. 94, no. 2, p. 85-104.

Vermeesch, P., 2004, How many grains are needed for a provenance study?: Earth and Planetary Science Letters, v. 224, p. 351-441.

Wintle, A. G., 1997, Luminescence dating: laboratory pro- cedures and protocols: Radiation Measurements, v. 27, p. 769-817.

Wyshnytzky, C. E., Rittenour, T. M., Nelson, M. S., and Thackray, G., 2015, Luminescence dating of late Pleistocene proximal glacial sediments in the Olympic Mountains, Washington: Quaternary International, v. 362, p. 116-123.

doi:10.1016/j.quaint.2014.08.024.

\section{Supplementary Figure Captions}

Figure S1. Microscope images of sands considered in this study. Photogarph taken of the 63-125 $\mu \mathrm{m}$ fraction. (A) Sample 13062101, Yarkand River (x35), (B) Sample 13062401, Hotan River (x40), Sample 13062403, Downstream Hotan River (x35), (D) Sample 13062503, Dune Sand (x50), (E) Sample 13062701, Kashgar River (x40).

Figure $S 2$. Equivalent dose $\left(D_{E}\right)$ distributions, calculated $D_{E}$ values, age model used for $D_{E}$ calculation ( $\mathrm{MAM}=$ minimum age model, $\mathrm{CAM}=$ central age model) and overdispersion (OD) values for OSL samples. Shaded region and filled points represents the 2-sigma range of the $\mathrm{D}_{\mathrm{E}}$ values used for age calculation. The MAM age model was used for samples with high OD $(>30 \%)$ and positive skew in the $\mathrm{D}_{\mathrm{E}}$ values, both indicative of partial bleaching (incomplete solar resetting).

Table $\mathrm{S} 1$. Analytical data for $\mathrm{U}-\mathrm{Pb}$ dating of detrital zircon grains from the river sediment samples. 
Table S2. Analytical data related to apatite fission track analysis of samples from the western Tarim Desert. 

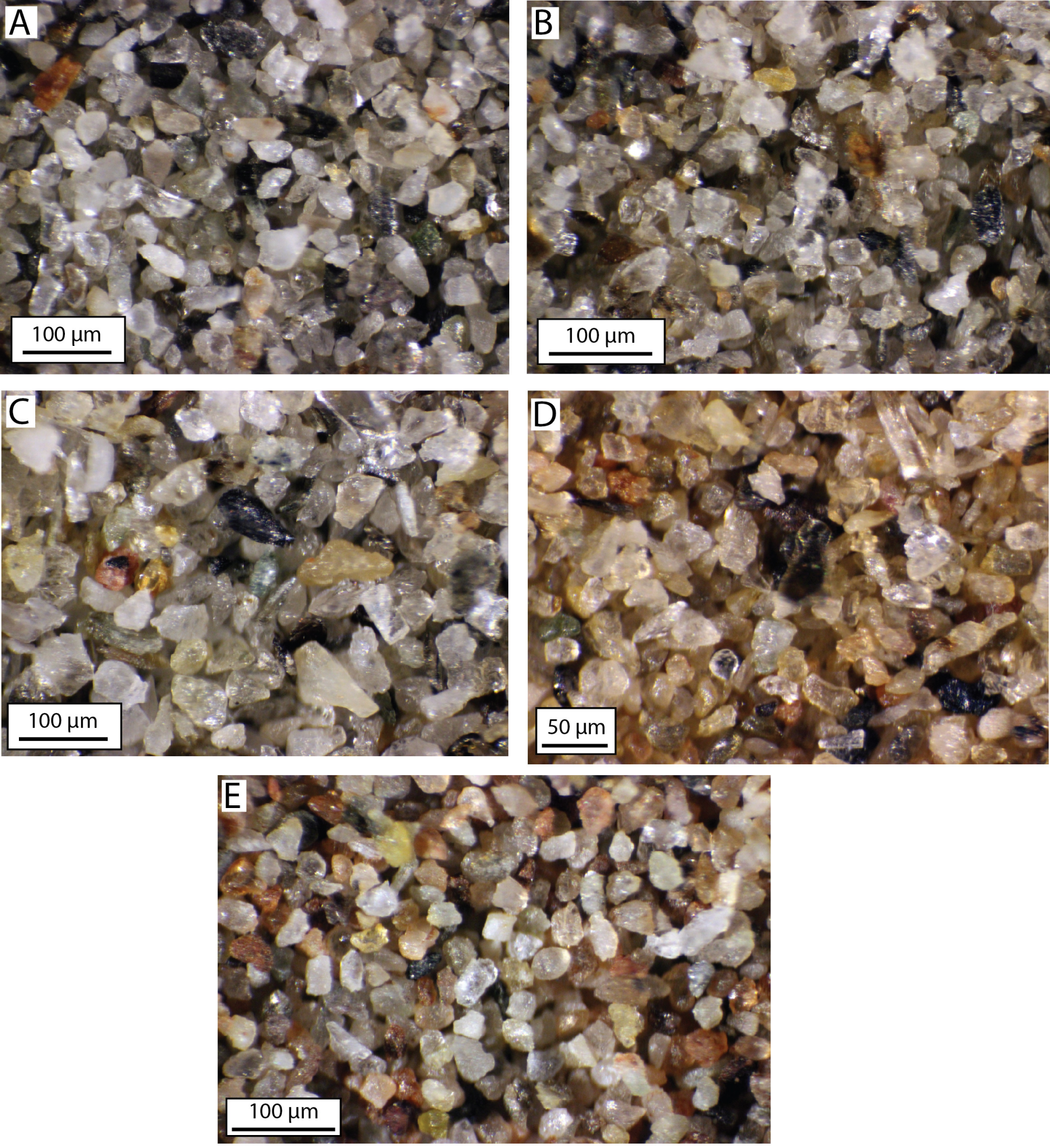

Figure S1

Clift et al. 


\section{Equivalent Dose $\left(D_{E}\right)$ Radial Plots}
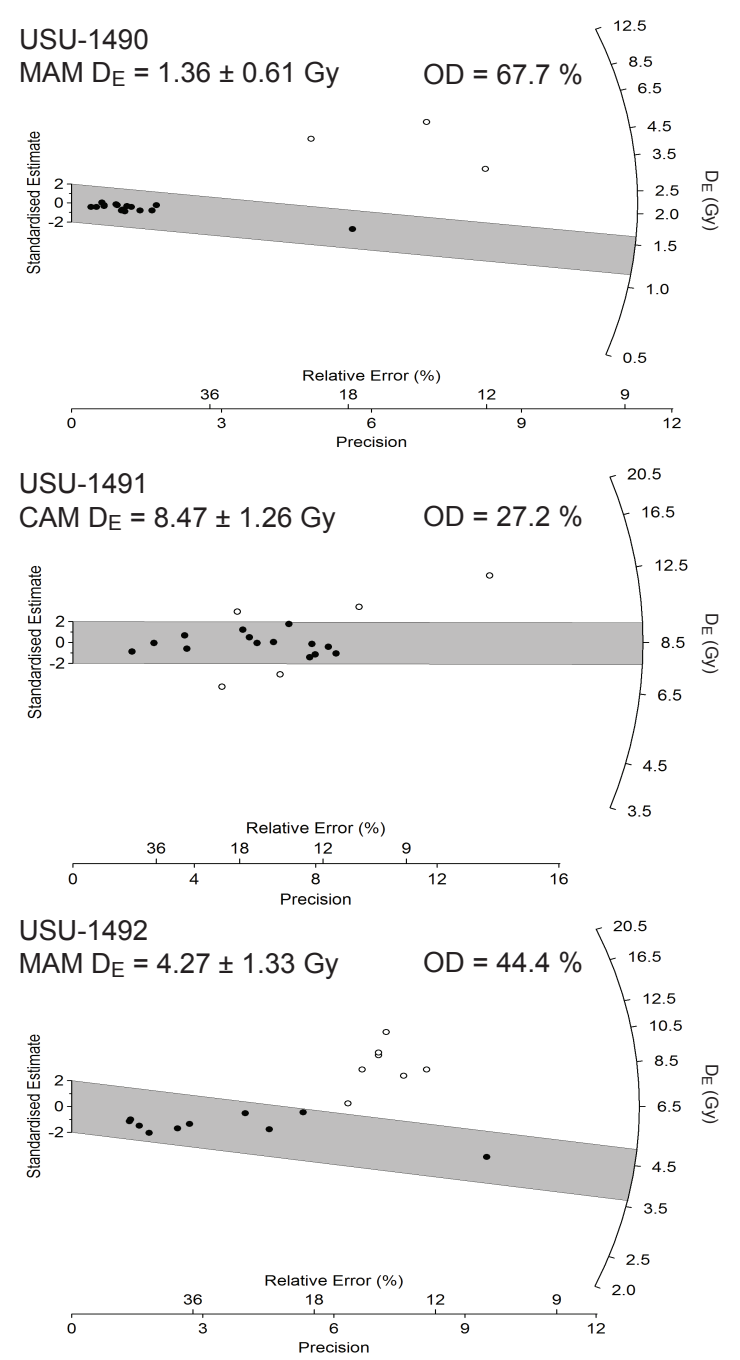
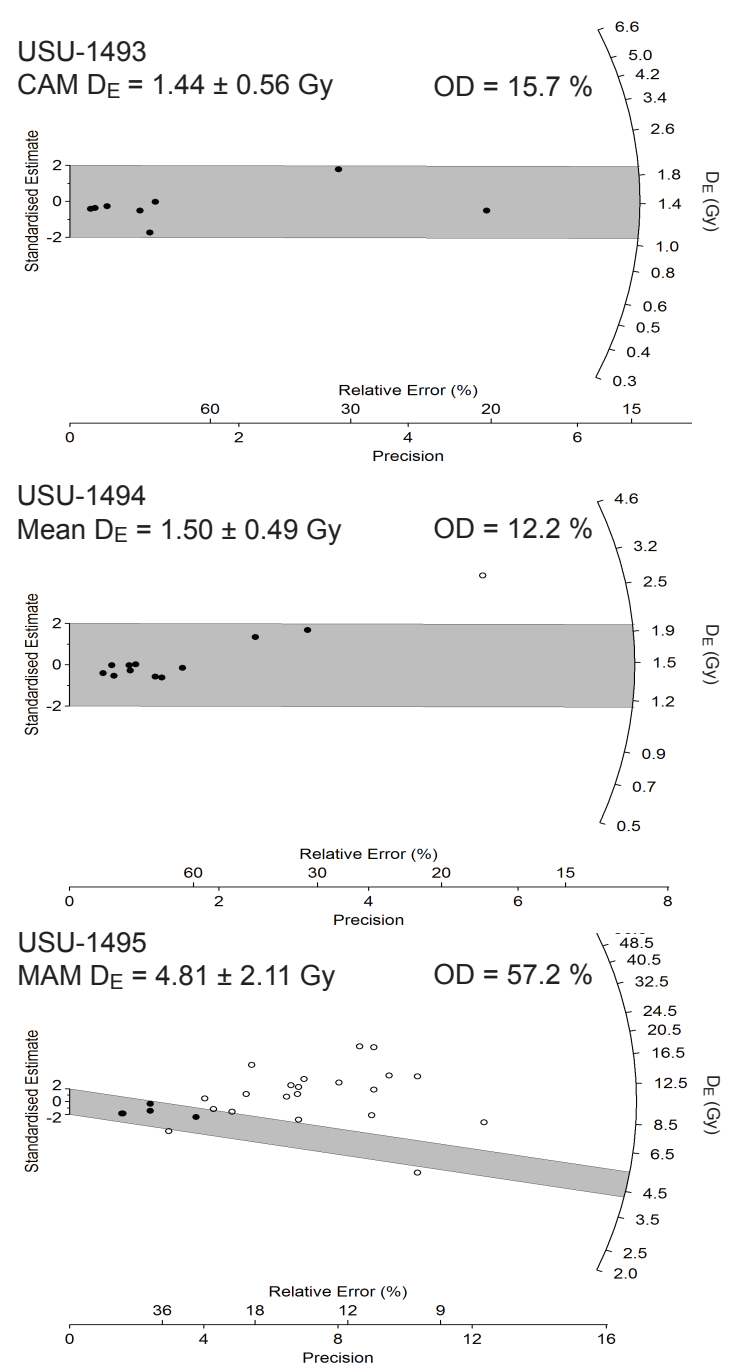

Figure S2

Clift et al. 
Ages (Ma)

$$
\text { U }
$$

Grain Pb (ppm) Atomic

No. (ppm)

Th/U 206/238

Sample 13062401 - Upper Hotan River

\begin{tabular}{|c|c|c|c|c|c|c|c|c|c|c|c|c|c|c|c|c|c|c|c|}
\hline G1 & 18.0 & 416.1 & 0.38 & 0.0416 & 0.0005 & 0.3017 & 0.0067 & 0.0526 & 0.0012 & 262.9 & 6.2 & 267.7 & 11.8 & 310.7 & 11.8 & 1.8 & 15.4 & 262.9 & 6.2 \\
\hline G2 & 16.6 & 483.9 & 0.49 & 0.0321 & 0.0004 & 0.2232 & 0.0052 & 0.0505 & 0.0012 & 203.4 & 4.9 & 204.6 & 9.7 & 218.5 & 9.1 & 0.6 & 6.9 & 203.4 & 4.9 \\
\hline G3 & 15.2 & 486.7 & 0.10 & 0.0332 & 0.0004 & 0.2250 & 0.0054 & 0.0492 & 0.0012 & 210.4 & 5.0 & 206.0 & 9.9 & 157.4 & 6.9 & -2.1 & -33.7 & 210.4 & 5.0 \\
\hline G4 & 11.5 & 376.2 & 0.17 & 0.0317 & 0.0004 & 0.2146 & 0.0072 & 0.0492 & 0.0017 & 200.9 & 5.2 & 197.4 & 13.0 & 155.9 & 9.8 & -1.8 & -28.8 & 200.9 & 5.2 \\
\hline G5 & 16.9 & 209.1 & 0.26 & 0.0813 & 0.0010 & 0.6190 & 0.0174 & 0.0552 & 0.0016 & 503.9 & 12.3 & 489.2 & 24.0 & 421.9 & 19.5 & -3.0 & -19.4 & 503.9 & 12.3 \\
\hline G6 & 13.7 & 421.7 & 0.17 & 0.0336 & 0.0005 & 0.2348 & 0.0094 & 0.0507 & 0.0021 & 213.3 & 5.9 & 214.2 & 16.5 & 224.9 & 16.3 & 0.4 & 5.2 & 213.3 & 5.9 \\
\hline G7 & 10.0 & 314.7 & 0.33 & 0.0313 & 0.0004 & 0.2131 & 0.0063 & 0.0494 & 0.0015 & 198.5 & 4.9 & 196.1 & 11.5 & 168.3 & 9.2 & -1.2 & -18.0 & 198.5 & 4.9 \\
\hline G8 & 14.8 & 474.2 & 0.21 & 0.0319 & 0.0004 & 0.2200 & 0.0054 & 0.0501 & 0.0012 & 202.3 & 4.9 & 201.9 & 10.0 & 197.8 & 8.8 & -0.2 & -2.3 & 202.3 & 4.9 \\
\hline G9 & 12.9 & 401.4 & 0.17 & 0.0335 & 0.0004 & 0.2326 & 0.0059 & 0.0505 & 0.0013 & 212.1 & 5.1 & 212.3 & 10.8 & 216.2 & 9.9 & 0.1 & 1.9 & 212.1 & 5.1 \\
\hline G10 & 14.5 & 360.9 & 0.83 & 0.0343 & 0.0004 & 0.2412 & 0.0071 & 0.0510 & 0.0015 & 217.5 & 5.5 & 219.4 & 12.7 & 239.9 & 12.6 & 0.8 & 9.3 & 217.5 & 5.5 \\
\hline G11 & 10.5 & 377.6 & 0.48 & 0.0259 & 0.0004 & 0.1819 & 0.0064 & 0.0510 & 0.0018 & 164.6 & 4.4 & 169.7 & 12.0 & 242.2 & 15.5 & 3.0 & 32.0 & 164.6 & 4.4 \\
\hline G12 & 131.2 & 1526.7 & 0.20 & 0.0873 & 0.0010 & 0.7742 & 0.0109 & 0.0644 & 0.0009 & 539.3 & 11.7 & 582.2 & 15.4 & 753.8 & 14.1 & 7.4 & 28.5 & 539.3 & 11.7 \\
\hline G13 & 92.6 & 269.2 & 0.83 & 0.2881 & 0.0034 & 4.5956 & 0.0750 & 0.1157 & 0.0018 & 1632.1 & 34.3 & 1748.5 & 33.1 & 1891.3 & 27.3 & 6.7 & 13.7 & 1891.3 & 27.3 \\
\hline G14 & 8.6 & 255.3 & 0.13 & 0.0335 & 0.0004 & 0.2269 & 0.0074 & 0.0491 & 0.0016 & 212.7 & 5.5 & 207.6 & 13.3 & 151.7 & 9.2 & -2.4 & -40.2 & 212.7 & 5.5 \\
\hline G15 & 18.1 & 597.9 & 0.09 & 0.0322 & 0.0004 & 0.2270 & 0.0051 & 0.0511 & 0.0012 & 204.5 & 4.9 & 207.7 & 9.6 & 245.3 & 9.8 & 1.5 & 16.7 & 204.5 & 4.9 \\
\hline G16 & 15.4 & 507.0 & 0.21 & 0.0311 & 0.0004 & 0.2150 & 0.0053 & 0.0501 & 0.0012 & 197.7 & 4.8 & 197.7 & 9.9 & 199.6 & 8.9 & 0.0 & 1.0 & 197.7 & 4.8 \\
\hline G17 & 4.5 & 121.7 & 0.88 & 0.0313 & 0.0005 & 0.2167 & 0.0103 & 0.0503 & 0.0024 & 198.6 & 5.8 & 199.2 & 18.3 & 206.6 & 18.1 & 0.3 & 3.9 & 198.6 & 5.8 \\
\hline G18 & 13.4 & 432.9 & 0.21 & 0.0318 & 0.0004 & 0.2196 & 0.0058 & 0.0502 & 0.0013 & 201.6 & 4.9 & 201.6 & 10.6 & 202.9 & 9.6 & 0.0 & 0.7 & 201.6 & 4.9 \\
\hline G19 & 18.5 & 535.0 & 0.40 & 0.0333 & 0.0004 & 0.2246 & 0.0053 & 0.0489 & 0.0012 & 211.2 & 5.0 & 205.7 & 9.8 & 144.0 & 6.3 & -2.7 & -46.7 & 211.2 & 5.0 \\
\hline G20 & 15.3 & 465.8 & 0.40 & 0.0318 & 0.0004 & 0.2240 & 0.0056 & 0.0512 & 0.0013 & 201.6 & 4.9 & 205.3 & 10.4 & 248.9 & 11.1 & 1.8 & 19.0 & 201.6 & 4.9 \\
\hline G21 & 9.1 & 283.9 & 0.30 & 0.0319 & 0.0004 & 0.2050 & 0.0069 & 0.0466 & 0.0016 & 202.4 & 5.2 & 189.3 & 12.5 & 30.8 & 2.0 & -6.9 & -556.3 & 202.4 & 5.2 \\
\hline G22 & 31.8 & 409.8 & 0.32 & 0.0766 & 0.0009 & 0.5968 & 0.0113 & 0.0566 & 0.0011 & 475.6 & 10.8 & 475.2 & 16.7 & 474.0 & 14.1 & -0.1 & -0.3 & 475.6 & 10.8 \\
\hline G23 & 30.1 & 1003.6 & 0.06 & 0.0323 & 0.0004 & 0.2180 & 0.0044 & 0.0490 & 0.0010 & 204.9 & 4.7 & 200.2 & 8.3 & 147.8 & 5.4 & -2.3 & -38.6 & 204.9 & 4.7 \\
\hline G24 & 63.1 & 305.6 & 0.96 & 0.1696 & 0.0020 & 1.7518 & 0.0302 & 0.0749 & 0.0013 & 1010.1 & 22.0 & 1027.8 & 26.6 & 1066.9 & 22.2 & 1.7 & 5.3 & 1066.9 & 22.2 \\
\hline G25 & 108.3 & 1572.1 & 0.43 & 0.0658 & 0.0008 & 0.4987 & 0.0082 & 0.0550 & 0.0009 & 410.5 & 9.2 & 410.8 & 13.3 & 413.8 & 10.9 & 0.1 & 0.8 & 410.5 & 9.2 \\
\hline G26 & 4.9 & 131.5 & 0.50 & 0.0376 & 0.0005 & 0.2542 & 0.0108 & 0.0491 & 0.0021 & 237.6 & 6.6 & 229.9 & 18.6 & 153.6 & 12.2 & -3.3 & -54.7 & 237.6 & 6.6 \\
\hline G27 & 7.6 & 240.6 & 0.24 & 0.0318 & 0.0004 & 0.2216 & 0.0076 & 0.0506 & 0.0018 & 201.8 & 5.2 & 203.2 & 13.7 & 220.8 & 13.7 & 0.7 & 8.6 & 201.8 & 5.2 \\
\hline G28 & 14.0 & 151.1 & 0.22 & 0.0945 & 0.0013 & 0.8316 & 0.0265 & 0.0639 & 0.0021 & 581.9 & 15.2 & 614.5 & 32.3 & 738.0 & 33.8 & 5.3 & 21.2 & 581.9 & 15.2 \\
\hline G29 & 12.6 & 410.5 & 0.13 & 0.0303 & 0.0004 & 0.2058 & 0.0058 & 0.0493 & 0.0014 & 192.6 & 4.8 & 190.0 & 10.7 & 159.7 & 8.3 & -1.3 & -20.5 & 192.6 & 4.8 \\
\hline G30 & 27.6 & 361.6 & 0.40 & 0.0734 & 0.0009 & 0.5744 & 0.0117 & 0.0568 & 0.0011 & 456.8 & 10.6 & 460.9 & 17.3 & 482.2 & 15.4 & 0.9 & 5.3 & 456.8 & 10.6 \\
\hline G31 & 90.2 & 1435.1 & 0.09 & 0.0668 & 0.0008 & 0.5142 & 0.0078 & 0.0559 & 0.0008 & 416.7 & 9.3 & 421.3 & 12.8 & 448.0 & 10.5 & 1.1 & 7.0 & 416.7 & 9.3 \\
\hline G32 & 265.4 & 3767.4 & 0.46 & 0.0665 & 0.0008 & 0.5061 & 0.0070 & 0.0552 & 0.0007 & 415.0 & 9.2 & 415.8 & 11.8 & 421.5 & 9.0 & 0.2 & 1.5 & 415.0 & 9.2 \\
\hline G33 & 15.7 & 550.4 & 0.09 & 0.0304 & 0.0004 & 0.2088 & 0.0052 & 0.0498 & 0.0012 & 193.2 & 4.6 & 192.6 & 9.7 & 186.1 & 8.5 & -0.3 & -3.8 & 193.2 & 4.6 \\
\hline G34 & 14.8 & 226.6 & 0.51 & 0.0609 & 0.0008 & 0.4723 & 0.0142 & 0.0563 & 0.0017 & 381.0 & 9.6 & 392.8 & 21.4 & 464.2 & 22.5 & 3.0 & 17.9 & 381.0 & 9.6 \\
\hline G35 & 78.4 & 2566.6 & 0.02 & 0.0333 & 0.0004 & 0.2269 & 0.0036 & 0.0494 & 0.0008 & 211.4 & 4.7 & 207.7 & 7.2 & 166.4 & 4.7 & -1.8 & -27.1 & 211.4 & 4.7 \\
\hline G36 & 40.7 & 536.4 & 0.57 & 0.0694 & 0.0008 & 0.5342 & 0.0099 & 0.0559 & 0.0010 & 432.2 & 9.9 & 434.6 & 15.3 & 448.4 & 13.1 & 0.5 & 3.6 & 432.2 & 9.9 \\
\hline G37 & 40.8 & 998.7 & 0.24 & 0.0400 & 0.0005 & 0.2913 & 0.0054 & 0.0529 & 0.0010 & 252.8 & 5.8 & 259.5 & 9.8 & 322.4 & 9.9 & 2.6 & 21.6 & 252.8 & 5.8 \\
\hline G38 & 89.1 & 752.5 & 0.49 & 0.1103 & 0.0013 & 0.9574 & 0.0148 & 0.0630 & 0.0009 & 674.3 & 14.7 & 682.0 & 18.6 & 708.9 & 15.1 & 1.1 & 4.9 & 674.3 & 14.7 \\
\hline
\end{tabular}

$\%$ concord. \% concord.

$\underline{(206 / 238} \quad \underline{(206 / 238}$ Best Age

207/235)

2

0

2

9

9

1 5 .7 .3

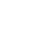

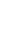
.0 .8 2 2 .2 6 9 4.7 
Ratios

$\mathbf{U}$
Ages (Ma)

Grain Pb (ppm) Atomic

No. (ppm) $\quad$ Th/U $206 / 238 \quad \pm$ s.e. $\quad 207 / 235 \quad \pm$ s.e. $\quad 207 / 206 \quad \pm$ s.e. $\quad 206 / 238 \quad \pm 2 s \quad 207 / 235 \quad \pm 2 s \quad 207 / 206 \quad+2 s$

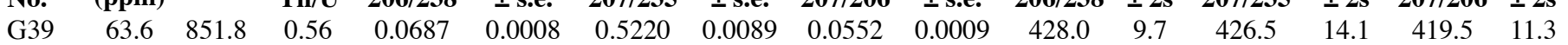

$\begin{array}{lllllllllllllllll}\text { G40 } & 33.4 & 1098.7 & 0.09 & 0.0324 & 0.0004 & 0.2309 & 0.0045 & 0.0518 & 0.0010 & 205.2 & 4.7 & 210.9 & 8.5 & 277.0 & 9.2\end{array}$

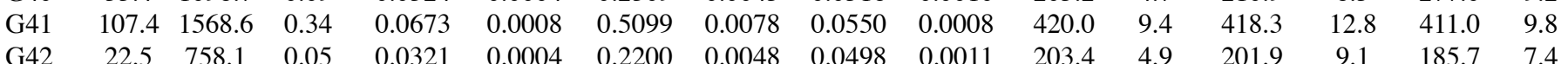

$\begin{array}{llllllllllllllll}\text { G42 } & 22.5 & 758.1 & 0.05 & 0.0321 & 0.0004 & 0.2200 & 0.0048 & 0.0498 & 0.0011 & 203.4 & 4.9 & 201.9 & 9.1 & 185.7 & 7.4 \\ \text { G43 } & 19.0 & 453.9 & 0.70 & 0.0367 & 0.0005 & 0.2523 & 0.0063 & 0.0499 & 0.0012 & 232.1 & 5.6 & 228.4 & 11.3 & 192.2 & 8.7\end{array}$

$\begin{array}{lllllllllllllllll}\text { G43 } & 19.0 & 453.9 & 0.70 & 0.0367 & 0.0005 & 0.2523 & 0.0063 & 0.0499 & 0.0012 & 232.1 & 5.6 & 228.4 & 11.3 & 192.2 & 8.7\end{array}$

$\begin{array}{cccccccccccccccc}\text { G44 } & 13.8 & 455.3 & 0.11 & 0.0320 & 0.0004 & 0.2229 & 0.0059 & 0.0506 & 0.0013 & 202.9 & 5.0 & 204.3 & 10.8 & 221.7 & 10.6 \\ \text { G45 } & 33.5 & 105.6 & 0.51 & 0.2889 & 0.0036 & 4.3289 & 0.0811 & 0.1088 & 0.0020 & 1635.8 & 35.7 & 1698.9 & 36.6 & 1779.1 & 30.9\end{array}$

$\begin{array}{llllllllllllllll}\text { G45 } & 33.5 & 105.6 & 0.51 & 0.2889 & 0.0036 & 4.3289 & 0.0811 & 0.1088 & 0.0020 & 1635.8 & 35.7 & 1698.9 & 36.6 & 1779.1 & 30.9 \\ \text { G46 } & 25.2 & 853.9 & 0.07 & 0.0318 & 0.0004 & 0.2221 & 0.0062 & 0.0508 & 0.0014 & 201.5 & 5.0 & 203.7 & 11.3 & 230.9 & 11.5\end{array}$

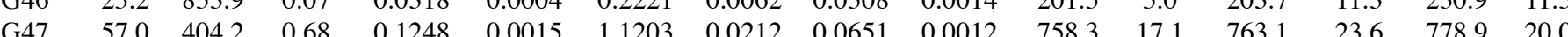

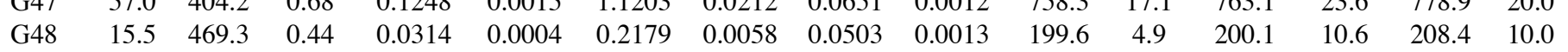

$\begin{array}{llllllllll}\mathrm{G} 49 & 23.9 & 827.3 & 0.03 & 0.0315 & 0.0004 & 0.2194 & 0.0047 & 0.0506 & 0.0011\end{array}$

$\begin{array}{llllllllll}\text { G50 } & 19.4 & 643.4 & 0.06 & 0.0326 & 0.0004 & 0.2265 & 0.0053 & 0.0505 & 0.0012\end{array}$

$\begin{array}{llllllllll}\text { G51 } & 36.2 & 455.3 & 0.76 & 0.0691 & 0.0008 & 0.5342 & 0.0107 & 0.0561 & 0.0011\end{array}$

$\begin{array}{llllllllll}\mathrm{G} 52 & 7.9 & 244.8 & 0.32 & 0.0318 & 0.0004 & 0.2196 & 0.0077 & 0.0502 & 0.0018\end{array}$

$\begin{array}{lllllllllll}\text { G53 } & 15.0 & 443.4 & 0.32 & 0.0334 & 0.0004 & 0.2343 & 0.0062 & 0.0509 & 0.0014\end{array}$

$\begin{array}{lccccccccc}\mathrm{G} 54 & 501.5 & 7936.9 & 0.07 & 0.0676 & 0.0008 & 0.5141 & 0.0073 & 0.0552 & 0.0007\end{array}$

$\begin{array}{lllllllllll}\text { G55 } & 11.5 & 338.5 & 0.40 & 0.0329 & 0.0004 & 0.2267 & 0.0070 & 0.0500 & 0.0016\end{array}$

$\begin{array}{llllllllll}\text { G56 } & 9.0 & 242.0 & 0.76 & 0.0324 & 0.0004 & 0.2293 & 0.0084 & 0.0514 & 0.0019\end{array}$

\begin{tabular}{llllllllll} 
G57 & 35.2 & 463.0 & 0.52 & 0.0708 & 0.0009 & 0.5510 & 0.0114 & 0.0565 & 0.0011 \\
\hline
\end{tabular}

$\begin{array}{lllllllllll}\text { G58 } & 144.0 & 1512.0 & 0.77 & 0.0840 & 0.0010 & 0.6831 & 0.0126 & 0.0590 & 0.0011\end{array}$

$\begin{array}{lllllllllll}\text { G59 } & 14.6 & 217.5 & 0.35 & 0.0658 & 0.0008 & 0.4937 & 0.0136 & 0.0545 & 0.0015\end{array}$

$\begin{array}{cccccccccc}\text { G60 } & 36.6 & 470.0 & 0.50 & 0.0728 & 0.0009 & 0.5548 & 0.0115 & 0.0553 & 0.0011 \\ \mathrm{G} 61 & 83.0 & 1444.9 & 1.19 & 0.0450 & 0.0005 & 0.3223 & 0.0058 & 0.0520 & 0.0009\end{array}$

$\begin{array}{llllllllll}\mathrm{G} 62 & 40.7 & 521.7 & 1.00 & 0.0639 & 0.0008 & 0.5098 & 0.0120 & 0.0579 & 0.0014\end{array}$

\begin{tabular}{llll}
$\mathrm{G} 63$ & 57.3 & 1241.3 & 0.50 \\
\hline
\end{tabular}

G64 $\quad 71.8 \quad 1110.6 \quad 0.35$

$\begin{array}{llll}\mathrm{G} 65 & 28.8 & 915.4 & 0.10\end{array}$

$\begin{array}{llll}\text { G66 } & 42.5 & 1356.7 & 0.15\end{array}$

G67 $\quad 31.1 \quad 824.5 \quad 0.49$

G68 $\quad 40.1 \quad 1272.1 \quad 0.01$

G69 $24.2 \quad 761.6 \quad 0.06$

G70 $\quad 146.7 \quad 1648.4 \quad 0.84$

$\begin{array}{llll}\text { G71 } & 23.8 & 337.1 & 0.56\end{array}$

G72 $40.6 \quad 1239.9 \quad 0.09$

$\begin{array}{llll}\mathrm{G} 73 & 10.3 & 233.6 & 0.54\end{array}$

$\begin{array}{llll}\text { G74 } & 44.0 & 585.4 & 0.37\end{array}$

$\begin{array}{llll}\mathrm{G} 75 & 43.5 & 81.8 & 0.94\end{array}$

$\begin{array}{llll}\text { G76 } & 79.0 & 2289.0 & 0.48\end{array}$

$\begin{array}{llll}\mathrm{G} 77 & 32.3 & 441.3 & 0.50\end{array}$ $\begin{array}{lllllll}0.0435 & 0.0005 & 0.3110 & 0.0058 & 0.0519 & 0.0009\end{array}$

0.0634

0.0335

0.0329

0.0357

$\begin{array}{llllll}0.0008 & 0.4919 & 0.0097 & 0.0564 & 0.0011\end{array}$

$\begin{array}{llllll}0.0004 & 0.2303 & 0.0051 & 0.0500 & 0.0011\end{array}$

$\begin{array}{lllll}0.0004 & 0.2288 & 0.0045 & 0.0505 & 0.0010 \\ 0.0005 & 0.2684 & 0.0072 & 0.0547 & 0.0015\end{array}$

$\begin{array}{llllll}0.0005 & 0.2684 & 0.0072 & 0.0547 & 0.0015\end{array}$

$\begin{array}{lllllll}0.0345 & 0.0004 & 0.2444 & 0.0049 & 0.0515 & 0.0010\end{array}$

0.076

$\begin{array}{lllll}0.0004 & 0.2417 & 0.0056 & 0.0512 & 0.0012 \\ 0.0009 & 0.5738 & 0.0099 & 0.0548 & 0.0009\end{array}$

$\begin{array}{llllll}0.0009 & 0.5738 & 0.0099 & 0.0548 & 0.0009\end{array}$

$\begin{array}{lllllll}0.0654 & 0.0008 & 0.4983 & 0.0115 & 0.0553 & 0.0013\end{array}$

0.0349

$\begin{array}{lllll}0.0004 & 0.2416 & 0.0047 & 0.0502 & 0.0010\end{array}$

$\begin{array}{lllll}0.0005 & 0.2839 & 0.0092 & 0.0503 & 0.0016\end{array}$

0.0729

0.4296

0.0328

$\begin{array}{lllll}0.0009 & 0.6003 & 0.0137 & 0.0597 & 0.0014 \\ 0.0053 & 9.2277 & 0.1559 & 0.1559 & 0.0025\end{array}$

$\begin{array}{lllll}0.0004 & 0.2269 & 0.0040 & 0.0503 & 0.0009\end{array}$

$\begin{array}{lllllll}0.0688 & 0.0008 & 0.5189 & 0.0110 & 0.0548 & 0.0011\end{array}$ $\begin{array}{llllll}199.8 & 4.7 & 201.4 & 8.9 & 222.2 & 8.4\end{array}$

$\begin{array}{llllll}206.6 & 5.0 & 207.3 & 9.8 & 218.1 & 9.1\end{array}$

$\begin{array}{llllll}430.6 & 10.0 & 434.6 & 16.3 & 457.5 & 14.4\end{array}$

$\begin{array}{llllll}201.5 & 5.4 & 201.6 & 13.8 & 205.2 & 13.1\end{array}$

$\begin{array}{llllll}211.9 & 5.2 & 213.8 & 11.3 & 236.3 & 11.2\end{array}$

$\begin{array}{llllll}421.6 & 9.4 & 421.2 & 12.1 & 420.3 & 9.1\end{array}$

$\begin{array}{llllll}208.8 & 5.4 & 207.5 & 12.6 & 194.1 & 11.0\end{array}$

$\begin{array}{llllll}205.4 & 5.5 & 209.6 & 14.9 & 259.7 & 16.8\end{array}$

$\begin{array}{llllll}441.2 & 10.2 & 445.6 & 17.0 & 470.5 & 15.2\end{array}$

$\begin{array}{llllll}519.9 & 11.9 & 528.7 & 17.8 & 568.6 & 15.6\end{array}$

$\begin{array}{llllll}410.5 & 10.2 & 407.4 & 20.4 & 391.8 & 17.9\end{array}$

$\begin{array}{llllll}8 & 10.6 & 448.1 & 17.1 & 425.2 & 14.1\end{array}$

$\begin{array}{llllll}399.4 & 9.6 & 418.3 & 18.1 & 526.4 & 19.2\end{array}$

$\begin{array}{llllll}274.5 & 6.4 & 275.0 & 10.5 & 281.0 & 8.9\end{array}$

$\begin{array}{llllll}396.1 & 9.2 & 406.2 & 15.2 & 466.2 & 14.5\end{array}$

$\begin{array}{llllll}212.1 & 5.1 & 210.5 & 9.5 & 194.5 & 7.6\end{array}$

$\begin{array}{llllll}208.4 & 4.9 & 209.2 & 8.5 & 219.9 & 7.6\end{array}$

$\begin{array}{llllll}225.8 & 5.6 & 241.4 & 12.6 & 397.9 & 17.5\end{array}$

$\begin{array}{llllll}218.4 & 5.1 & 222.0 & 9.1 & 262.4 & 9.0\end{array}$

$\begin{array}{llllll}217.2 & 5.2 & 219.8 & 10.3 & 249.8 & 10.1\end{array}$

$\begin{array}{llllll}472.0 & 10.8 & 460.5 & 15.1 & 405.3 & 11.1\end{array}$

$\begin{array}{llllll}408.1 & 9.8 & 410.6 & 17.5 & 426.0 & 15.8\end{array}$

$\begin{array}{llllll}259.1 & 6.7 & 253.8 & 15.8 & 207.0 & 12.2\end{array}$

$\begin{array}{llllll}453.8 & 10.8 & 477.4 & 19.6 & 594.2 & 20.3\end{array}$

$\begin{array}{lllllll}2303.9 & 47.3 & 2360.9 & 37.1 & 2412.0 & 29.8\end{array}$

$\begin{array}{llllll}207.8 & 4.9 & 207.7 & 7.8 & 208.4 & 6.4\end{array}$

$\begin{array}{llllll}428.7 & 10.1 & 424.4 & 16.6 & 403.3 & 13.7\end{array}$
$\%$ concord. \% concord.

$\underline{(206 / 238} \quad \underline{(206 / 238}$ Best Age

207/235) 207/206) (Ma) $\pm 2 s$

$\begin{array}{llll}-0.4 & -2.0 & 428.0 & 9.7\end{array}$

$\begin{array}{lll}-2.2-420.0 & 9.4\end{array}$

$\begin{array}{llll}-0.8 & -9.6 & 203.4 & 4.9\end{array}$

$\begin{array}{llll}-1.6 & -20.8 & 232.1 & 5.6\end{array}$

$\begin{array}{lll}-20.8 & 232.9 & 5.6\end{array}$

$\begin{array}{llll}3.7 & 8.1 & 1779.1 & 30.9\end{array}$

$\begin{array}{llll}1.1 & 12.7 & 201.5 & 5.0\end{array}$

$\begin{array}{llll}0.6 & 2.6 & -758.3 & 17.1\end{array}$

$\begin{array}{llll}0.3 & 4.3 & 199.6 & 4.9\end{array}$

$\begin{array}{llll}0.8 & 10.1 & 199.8 & 4.7\end{array}$

$206.6 \quad 5.0$

$\begin{array}{llll}0.9 & 5.9 & 430.6 & 10.0\end{array}$

$\begin{array}{llll}0.1 & 1.8 & 201.5 & 5.4\end{array}$

$\begin{array}{llll}0.9 & 10.3 & 211.9 & 5.2\end{array}$

$\begin{array}{llll}-0.1 & -0.3 & 421.6 & 9.4\end{array}$

$\begin{array}{llll}-0.6 & -7.6 & 208.8 & 5.4\end{array}$

$\begin{array}{llll}2.0 & 20.9 & 205.4 & 5.5\end{array}$

$\begin{array}{llll}1.0 & 6.2 & 441.2 & 10.2\end{array}$

$\begin{array}{llll}1.7 & 8.6 & 519.9 & 11.9\end{array}$

$\begin{array}{llll}-0.8 & -4.8 & 410.5 & 10.2\end{array}$

$\begin{array}{llll}-1.1 & -6.5 & 453.0 & 10.6\end{array}$

$\begin{array}{llll}0.0 & 0.4 & 283.8 & 6.5\end{array}$

$\begin{array}{llll}4.5 & 24.1 & 399.4 & 9.6\end{array}$

$\begin{array}{llll}0.2 & 2.3 & 274.5 & 6.4\end{array}$

$\begin{array}{llll}2.5 & 15.0 & 396.1 & 9.2\end{array}$

$\begin{array}{llll}-0.8 & -9.0 & 212.1 & 5.1\end{array}$

$\begin{array}{llll}0.4 & 5.2 & 208.4 & 4.9\end{array}$

$\begin{array}{llll}6.5 & 43.3 & 225.8 & 5.6\end{array}$

$\begin{array}{llll}1.6 & 16.8 & 218.4 & 5.1\end{array}$

$\begin{array}{llll}1.2 & 13.1 & 217.2 & 5.2\end{array}$

$\begin{array}{llll}-2.5 & -16.5 & 472.0 & 10.8\end{array}$

$\begin{array}{llll}0.6 & 4.2 & 408.1 & 9.8\end{array}$

$\begin{array}{llll}-0.7 & -7.8 & 221.3 & 5.2\end{array}$

$\begin{array}{llll}-2.1 & -25.1 & 259.1 & 6.7\end{array}$

$\begin{array}{llll}4.9 & 23.6 & 453.8 & 10.8\end{array}$

$\begin{array}{llll}2.4 & 4.5 & 2412.0 & 29.8\end{array}$

$\begin{array}{llll}-0.1 & 0.3 & 207.8 & 4.9\end{array}$

$\begin{array}{llll}-1.0 & -6.3 & 428.7 & 10.1\end{array}$ 
Table S1

Ratios

$\mathbf{U}$

Grain Pb (ppm) Atomic

No. (ppm) $\mathbf{T h} / \mathbf{U}$

$\begin{array}{llll}\text { G78 } & 32.8 & 622.4 & 0.83\end{array}$

$\begin{array}{llll}\text { G79 } & 256.3 \quad 4018.5 & 0.05\end{array}$

$\begin{array}{llll}\text { G80 } & 16.9 & 492.3 & 0.12\end{array}$

$\begin{array}{llll}\text { G81 } & 295.0 & 9720.2 & 0.01\end{array}$

$\begin{array}{llll}\text { G82 } & 27.3 & 910.6 & 0.07\end{array}$

$\begin{array}{llll}\text { G83 } & 3.8 & 76.2 & 0.64\end{array}$

$\begin{array}{llll}\text { G84 } & 24.8 & 355.3 & 0.54\end{array}$

$\begin{array}{llll}\text { G85 } & 33.7 & 511.9 & 0.23\end{array}$

$\begin{array}{llll}\text { G86 } & 130.0 & 1621.1 & 0.89\end{array}$

G87 $\quad 311.3 \quad 4611.5 \quad 0$.

$\begin{array}{llll}\text { G88 } & 10.9 & 350.4 & 0.16\end{array}$

$\begin{array}{llll}\text { G89 } & 81.0 & 985.4 & 0.76\end{array}$

G90 $23.9 \quad 105.6 \quad 0.41$

$\begin{array}{llll}\text { G91 } & 54.9 & 833.6 & 0.23\end{array}$

$\begin{array}{llll}\text { G92 } & 11.7 & 159.5 & 0.53\end{array}$

$\begin{array}{llll}\text { G93 } & 114.2 & 1521.8 & 0.56\end{array}$

G94 $\quad 84.0 \quad 963.7 \quad 0.40$

$\begin{array}{llll}\text { G95 } & 41.6 & 194.4 & 0.97\end{array}$

$\begin{array}{llll}\text { G96 } & 17.3 & 563.7 & 0.14\end{array}$

$\begin{array}{llll}\text { G97 } & 85.1 & 1300.8 & 0.18\end{array}$

$\begin{array}{llll}\text { G98 } & 63.6 & 2057.5 & 0.05\end{array}$

$\begin{array}{llll}\text { G99 } & 36.8 & 1211.3 & 0.07\end{array}$

$\begin{array}{llll}\mathrm{G} 100 & 66.2 & 880.5 & 0.25\end{array}$

$\begin{array}{llll}\mathrm{G} 101 & 20.7 & 604.2 & 0.49\end{array}$

$\begin{array}{llll}\mathrm{G} 102 & 9.9 & 281.8 & 0.51\end{array}$

$\begin{array}{llll}\mathrm{G} 103 & 22.7 & 693.1 & 0.13\end{array}$

G104 $\quad 148.5 \quad 2152.6 \quad 0.37$

$\begin{array}{llll}\text { G105 } & 33.5 & 1132.2 & 0.07\end{array}$

$\begin{array}{llll}\text { G106 } & 41.9 & 1258.1 & 0.35\end{array}$

$\begin{array}{llll}\text { G107 } & 27.7 & 573.5 & 0.39\end{array}$

$\begin{array}{llll}\mathrm{G} 108 & 32.3 & 92.3 & 0.59\end{array}$

$\begin{array}{llll}\text { G109 } & 15.9 & 307.7 & 1.07\end{array}$

$\begin{array}{llll}\text { G110 } & 56.0 & 1919.7 & 0.05\end{array}$

$\begin{array}{llll}\text { G111 } & 106.8 & 1633.0 & 0.27\end{array}$

$\begin{array}{llll}\mathrm{G} 112 & 62.8 & 786.8 & 0.83\end{array}$

$\begin{array}{llll}\text { G113 } & 39.9 & 528.7 & 0.48\end{array}$

$\begin{array}{llll}\text { G114 } & 21.2 \quad 684.7 \quad 0.08\end{array}$

$\begin{array}{llll}\text { G115 } & 69.9 & 539.9 & 0.72\end{array}$

$\begin{array}{llll}\text { G116 } & 11.2 \quad 344.1 \quad 0.17\end{array}$

0.0322

0.0667

0.0324

0.0719

0.0672

0.0687

0.0695

0.1763

0.0321

0.0676

0.0327

0.0759

0.0330

0.0342

0.0332

0.0468
0.3120

0.0416

0.0685

0.1135
$206 / 238 \pm$ s.e. $\quad 207 / 235 \quad \pm$ s.e. $207 / 206 \quad \pm$ s.e. $206 / 238 \quad \pm 2 s \quad 207 / 235 \quad \pm 2 s \quad 207 / 206 \quad \pm 2 s$ $\begin{array}{llllllllllll}0.0452 & 0.0006 & 0.3205 & 0.0071 & 0.0515 & 0.0011 & 285.0 & 6.8 & 282.3 & 12.3 & 262.4 & 10.1\end{array}$ $\begin{array}{lllllll}0.0686 & 0.0008 & 0.5293 & 0.0082 & 0.0560 & 0.0008 & 427.6\end{array}$

$\begin{array}{llllll}0.0347 & 0.0004 & 0.2402 & 0.0062 & 0.0502 & 0.0013\end{array}$

$\begin{array}{llllll}0.0333 & 0.0004 & 0.2331 & 0.0036 & 0.0509 & 0.0007\end{array}$

$\begin{array}{llllll}0.0452 & 0.0008 & 0.3195 & 0.0191 & 0.0514 & 0.0031\end{array}$

$\begin{array}{llllll}0.0647 & 0.0008 & 0.5052 & 0.0135 & 0.0567 & 0.0015\end{array}$

0.0675

$\begin{array}{llllll}0.0658 & 0.0008 & 0.5058 & 0.0100 & 0.0558 & 0.0011\end{array}$

$\begin{array}{lllllll}0.0714 & 0.0009 & 0.5705 & 0.0147 & 0.0580 & 0.0015\end{array}$

$\begin{array}{lllllll}0.0332 & 0.0004 & 0.2324 & 0.0058 & 0.0508 & 0.0013\end{array}$

$\begin{array}{lllll}0.0014 & 1.0615 & 0.0234 & 0.0679 & 0.0015\end{array}$

0.0323

$\begin{array}{lllll}0.0004 & 0.2266 & 0.0072 & 0.0509 & 0.0016\end{array}$ $\begin{array}{llllll}427.6 & 9.7 & 431.3 & 13.1 & 453.2 & 10.6\end{array}$

$\begin{array}{llllll}220.0 & 5.5 & 218.6 & 11.3 & 205.7 & 9.6\end{array}$

$\begin{array}{llllll}210.9 & 4.9 & 212.8 & 7.2 & 236.3 & 6.1\end{array}$

$\begin{array}{llllll}204.4 & 4.9 & 208.8 & 9.2 & 261.0 & 9.8\end{array}$

$\begin{array}{llllll}284.7 & 9.5 & 281.5 & 31.0 & 256.6 & 27.6\end{array}$

$\begin{array}{llllll}403.9 & 10.1 & 415.2 & 20.2 & 480.3 & 20.4\end{array}$

$\begin{array}{llllll}416.2 & 9.8 & 416.0 & 16.1 & 416.7 & 13.8\end{array}$

$\begin{array}{llllll}424.1 & 9.8 & 429.3 & 14.4 & 459.5 & 12.4\end{array}$

$\begin{array}{llllll}433.4 & 9.9 & 420.8 & 13.3 & 354.3 & 9.2\end{array}$

$\begin{array}{llllll}205.7 & 5.4 & 220.3 & 13.9 & 380.6 & 20.6\end{array}$

$\begin{array}{llllll}447.3 & 10.5 & 480.5 & 17.6 & 643.8 & 18.6\end{array}$

$\begin{array}{llllll}1219.1 & 27.9 & 1220.3 & 34.7 & 1224.1 & 29.8\end{array}$

$\begin{array}{llllll}419.4 & 9.8 & 421.5 & 15.1 & 435.2 & 12.9\end{array}$

$\begin{array}{llllll}428.2 & 11.6 & 430.7 & 27.0 & 446.0 & 25.9\end{array}$

$\begin{array}{llllll}433.3 & 10.0 & 435.7 & 14.4 & 450.4 & 12.0\end{array}$

$\begin{array}{llllll}524.8 & 12.1 & 544.5 & 18.0 & 630.0 & 16.5\end{array}$

$\begin{array}{llllll}046.7 & 23.7 & 1062.8 & 30.0 & 1097.7 & 25.7\end{array}$

$\begin{array}{lllllll}203.7 & 5.1 & 208.7 & 10.9 & 267.3 & 12.1\end{array}$

$\begin{array}{llllll}421.6 & 9.8 & 424.4 & 14.5 & 442.0 & 12.2\end{array}$

$\begin{array}{llllll}212.3 & 5.0 & 215.6 & 8.4 & 253.9 & 8.1\end{array}$

$\begin{array}{llllll}207.2 & 5.0 & 210.1 & 9.0 & 244.9 & 8.9\end{array}$

$\begin{array}{llllll}471.5 & 11.1 & 481.2 & 18.1 & 529.8 & 16.7\end{array}$

$\begin{array}{llllll}204.7 & 5.1 & 210.6 & 10.8 & 279.7 & 12.4\end{array}$

$\begin{array}{llllll}209.0 & 5.5 & 213.4 & 14.0 & 264.2 & 15.7\end{array}$

$\begin{array}{llllll}216.6 & 5.4 & 219.8 & 10.6 & 255.7 & 10.7\end{array}$

$\begin{array}{llllll}421.0 & 9.7 & 423.1 & 14.1 & 436.4 & 11.7\end{array}$

$\begin{array}{llllll}202.4 & 4.9 & 205.3 & 9.1 & 239.9 & 9.1\end{array}$

$\begin{array}{llllll}210.3 & 5.0 & 214.5 & 9.2 & 262.4 & 9.4\end{array}$

$\begin{array}{llllll}295.1 & 7.1 & 302.4 & 13.5 & 361.4 & 13.8\end{array}$

$\begin{array}{llllll}1750.4 & 38.4 & 1834.8 & 38.4 & 1933.6 & 32.5\end{array}$

$\begin{array}{llllll}262.7 & 6.7 & 276.8 & 15.3 & 399.2 & 19.0\end{array}$

$\begin{array}{llllll}200.6 & 4.7 & 204.4 & 8.3 & 250.3 & 8.4\end{array}$

$\begin{array}{llllll}410.6 & 9.7 & 415.6 & 15.3 & 445.2 & 13.6\end{array}$

$\begin{array}{llllll}427.3 & 10.1 & 442.9 & 17.3 & 526.7 & 17.0\end{array}$

$\begin{array}{llllll}444.5 & 11.1 & 458.3 & 21.1 & 530.1 & 21.1\end{array}$

$\begin{array}{llllll}210.6 & 5.2 & 212.2 & 10.6 & 231.8 & 10.2\end{array}$

$\begin{array}{llllll}693.0 & 16.4 & 734.5 & 25.9 & 864.9 & 24.9\end{array}$

$\begin{array}{llllll}205.0 & 5.4 & 207.3 & 12.9 & 235.9 & 13.3\end{array}$
$\%$ concord. \% concord.

$\underline{(206 / 238} \quad \underline{(206 / 238}$ Best Age

207/235) 207/206) (Ma) $\pm 2 \mathrm{~s}$

$\begin{array}{llll}-1.0 & -8.6 & 285.0 & 6.8\end{array}$

$\begin{array}{llll}0.9 & 5.6 & 427.6 & 9.7\end{array}$

$\begin{array}{llll}-0.7 & -7.0 & 220.0 & 5.5\end{array}$

$\begin{array}{llll}0.9 & 10.8 & 210.9 & 4.9\end{array}$

$\begin{array}{llll}2.1 & 21.7 & 204.4 & 4.9\end{array}$

$\begin{array}{llll}-1.2 & -11.0 & 284.7 & 9.5\end{array}$

$\begin{array}{llll}2.7 & 15.9 & 403.9 & 10.1\end{array}$

$\begin{array}{llll}-0.1 & 0.1 & 416.2 & 9.8\end{array}$

$\begin{array}{llll}1.2 & 7.7 & 424.1 & 9.8\end{array}$

$\begin{array}{llll}-3.0 & -22.3 & 433.4 & 9.9\end{array}$

$\begin{array}{llll}6.6 & 45.9 & 205.7 & 5.4\end{array}$

$\begin{array}{llll}6.9 & 30.5 & 447.3 & 10.5\end{array}$

$\begin{array}{llll}0.1 & 0.4 & 1224.1 & 29.8\end{array}$

$\begin{array}{llll}0.5 & 3.6 & 419.4 & 9.8\end{array}$

$\begin{array}{llll}0.6 & 4.0 & 428.2 & 11.6\end{array}$

$\begin{array}{llll}0.5 & 3.8 & 433.3 & 10.0\end{array}$

$\begin{array}{llll}3.6 & 16.7 & 524.8 & 12.1\end{array}$

$\begin{array}{llll}1.5 & 4.6 & 1097.7 & 25.7\end{array}$

$\begin{array}{llll}2.4 & 23.8 & 203.7 & 5.1\end{array}$

$\begin{array}{llll}0.7 & 4.6 & 421.6 & 9.8\end{array}$

$\begin{array}{llll}1.5 & 16.4 & 212.3 & 5.0\end{array}$

$\begin{array}{llll}1.4 & 15.4 & 207.2 & 5.0\end{array}$

$\begin{array}{llll}2.0 & 11.0 & 471.5 & 11.1\end{array}$

$\begin{array}{llll}2.8 & 26.8 & 204.7 & 5.1\end{array}$

$\begin{array}{llll}2.0 & 20.9 & 209.0 & 5.5\end{array}$

$\begin{array}{llll}1.4 & 15.3 & 216.6 & 5.4\end{array}$

$\begin{array}{llll}0.5 & 3.5 & 421.0 & 9.7\end{array}$

$\begin{array}{llll}1.4 & 15.6 & 202.4 & 4.9\end{array}$

$\begin{array}{llll}1.9 & 19.9 & 210.3 & 5.0\end{array}$

$\begin{array}{llll}2.4 & 18.4 & 295.1 & 7.1\end{array}$

$\begin{array}{llll}4.6 & 9.5 & 1933.6 & 32.5\end{array}$

$\begin{array}{llll}5.1 & 34.2 & 262.7 & 6.7\end{array}$

$\begin{array}{llll}1.9 & 19.8 & 200.6 & 4.7\end{array}$

$\begin{array}{llll}1.2 & 7.8 & 410.6 & 9.7\end{array}$

$\begin{array}{llll}3.5 & 18.9 & 427.3 & 10.1\end{array}$

$\begin{array}{llll}3.0 & 16.1 & 444.5 & 11.1\end{array}$

$\begin{array}{llll}0.8 & 9.1 & 210.6 & 5.2\end{array}$

$\begin{array}{llll}5.6 & 19.9 & 693.0 & 16.4\end{array}$

$\begin{array}{llll}1.1 & 13.1 & 205.0 & 5.4\end{array}$ 
Ratios$$
\mathbf{U}
$$

Grain Pb (ppm) Atomic

$\mathbf{T h} / \mathrm{U} \quad 206$

G117 $\quad 188.4 \quad 2707.2 \quad 0.62$

$\begin{array}{llll}\mathrm{G} 118 & 62.0 & 1200.1 & 0.87\end{array}$

$\begin{array}{llll}\text { G119 } & 15.7 & 304.9 & 0.63\end{array}$

$\begin{array}{llll}\text { G120 } & 83.9 & 1175.6 & 0.35\end{array}$

$\begin{array}{llll}\text { G121 } & 140.4 & 442.7 & 0.88\end{array}$

$\begin{array}{llll}\mathrm{G} 122 & 32.3 & 464.4 & 0.38\end{array}$

$\begin{array}{llll}\mathrm{G} 123 & 91.8 & 1384.0 & 0.13\end{array}$

G124 $47.2 \quad 954.6 \quad 0.53$

$\begin{array}{llll}\text { G125 } & 12.2 & 378.3 & 0.38\end{array}$

$\begin{array}{llll}\text { G126 } & 36.6 & 1158.8 & 0.17\end{array}$

\begin{tabular}{cccc}
$G 127$ & 13.1 & 376.9 & 0.29 \\
\hline & 57.7 & 1884.0 & 0.03
\end{tabular}

$\begin{array}{llll}\mathrm{G} 129 & 131.5 & 186.7 & 1.22\end{array}$

$\begin{array}{llll}\mathrm{G} 130 & 19.5 & 162.2 & 0.42\end{array}$

$\begin{array}{llll}\text { G131 } & 20.4 & 283.2 & 0.44\end{array}$

$\begin{array}{llll}\text { G132 } & 70.4 & 1065.1 & 0.1\end{array}$

$\begin{array}{llcc}\text { Sample 13062402 - Dune Sand } & \text { 13.3 } & 202.1 & 0.54 \\ \text { Sam } & \end{array}$

\begin{tabular}{llll} 
& 10.6 & 281.1 & 0.63 \\
\hline & 81.2 & 738.5 & 0.45
\end{tabular}

$\begin{array}{llll}\text { G3 } & 447.0 & 6606.0 & 0.23\end{array}$

$\begin{array}{llll}\text { G4 } & 23.5 & 620.3 & 0.27\end{array}$

$\begin{array}{llll}\text { G5 } & 63.0 & 759.5 & 0.70\end{array}$

$\begin{array}{llll}\text { G6 } & 74.8 & 833.6 & 0.82\end{array}$

G7 $\quad 14.5 \quad 330.8 \quad 0.45$

$\begin{array}{llll}\text { G8 } & 20.5 & 400.7 & 0.62\end{array}$

$\begin{array}{llll}\text { G9 } & 44.2 & 871.4 & 0.58\end{array}$

$\begin{array}{llll}\mathrm{G} 10 & 98.8 & 183.2 & 1.60\end{array}$

$\begin{array}{llll}\text { G11 } & 168.5 & 341.3 & 0.34\end{array}$

$\begin{array}{llll}\mathrm{G} 12 & 72.3 & 265.1 & 0.53\end{array}$

$\begin{array}{llll}\text { G13 } & 91.4 & 1256.7 & 0.42\end{array}$

$\begin{array}{llll}\text { G14 } & 48.0 & 1183.3 & 0.72\end{array}$

$\begin{array}{llll}\text { G15 } & 49.5 & 632.2 & 0.49\end{array}$

$\begin{array}{llll}\text { G16 } & 54.1 & 694.5 & 0.38\end{array}$

G17 $\quad 70.1 \quad 871.4 \quad 0.40$

$\begin{array}{llll}\text { G18 } & 26.6 \quad 532.2 & 0.52\end{array}$

$\begin{array}{llll}\text { G19 } & 83.0 & 1149.0 & 0.37\end{array}$

$\begin{array}{llll}\mathrm{G} 20 & 18.2 & 258.1 & 0.38\end{array}$

G21

0.0700

0.2662

0.0464

0.0349

0.0334

0.5223

0.0692

0.0691

0.0638

0.001

0.0342

0.0686

0.0731

0.0417

0.0470

0.3766

0.4527

0.0696

0.0358

0.0732

0.0774

0.0463

0.0704 $\mathbf{2 0 6} / \mathbf{2 3 8} \pm$ s.e. $207 / 235 \pm$ s.e. $207 / 206 \pm$ s.e. $206 / 238 \pm 2$ s $207 / 235$

$\begin{array}{llllll}0.0006 & 0.3523 & 0.0101 & 0.0546 & 0.0016\end{array}$

$\begin{array}{llllll}0.0009 & 0.5414 & 0.0106 & 0.0561 & 0.0010\end{array}$

$\begin{array}{lllll}0.0032 & 3.7493 & 0.0683 & 0.1022 & 0.0018\end{array}$

$\begin{array}{lllll}0.0009 & 0.5508 & 0.0125 & 0.0591 & 0.0013\end{array}$

$\begin{array}{lllll}0.0005 & 0.2206 & 0.0097 & 0.0509 & 0.0023 \\ 0.0004 & 0.2299 & 0.0054 & 0.0507 & 0.0011\end{array}$

$\begin{array}{lllll}0.0005 & 0.2522 & 0.0097 & 0.0525 & 0.0020\end{array}$

$\begin{array}{lllll}0.0004 & 0.2355 & 0.0055 & 0.0512 & 0.0012 \\ 0.0065 & 13.1355 & 0.2524 & 0.1825 & 0.0033\end{array}$

$\begin{array}{ccccc}0.0065 & 13.1355 & 0.2524 & 0.1825 & 0.0033 \\ 0.0016 & 1.0852 & 0.0341 & 0.0687 & 0.0022\end{array}$

$\begin{array}{lllll}0.0009 & 0.5417 & 0.0162 & 0.0568 & 0.0017\end{array}$

$\begin{array}{lllll}0.0009 & 0.5384 & 0.0112 & 0.0565 & 0.0011\end{array}$

$\begin{array}{lllll}0.0005 & 0.2515 & 0.0107 & 0.0534 & 0.0023\end{array}$

$\begin{array}{lllll}0.0013 & 0.8922 & 0.0189 & 0.0621 & 0.0013\end{array}$

$\begin{array}{lllll}0.0005 & 0.2687 & 0.0063 & 0.0513 & 0.0012\end{array}$

$\begin{array}{lllll}0.0009 & 0.5576 & 0.0100 & 0.0554 & 0.0010\end{array}$

$\begin{array}{lllllll}0.0767 & 0.0009 & 0.5954 & 0.0104 & 0.0563 & 0.0009\end{array}$

\begin{tabular}{llllll}
0.0005 & 0.2961 & 0.0083 & 0.0515 & 0.0014 \\
\hline
\end{tabular}

$\begin{array}{lllll}.0 .006 & 0.3376 & 0.0083 & 0.0529 & 0.0013\end{array}$

$\begin{array}{lllll}0.0006 & 0.3468 & 0.0069 & 0.0535 & 0.0010 \\ 0.0047 & 7.8954 & 0.1302 & 0.1521 & 0.0024\end{array}$

$\begin{array}{llllll}0.0054 & 10.0818 & 0.1420 & 0.1616 & 0.0021\end{array}$

$\begin{array}{llllll}0.0030 & 3.1026 & 0.0493 & 0.0909 & 0.0014\end{array}$

$\begin{array}{lllll}0.0008 & 0.5348 & 0.0087 & 0.0557 & 0.0009 \\ 0.0005 & 0.2566 & 0.0064 & 0.0519 & 0.0013\end{array}$

$\begin{array}{lllll}0.0009 & 0.5627 & 0.0105 & 0.0558 & 0.0010\end{array}$

$\begin{array}{lllll}0.0009 & 0.5834 & 0.0104 & 0.0562 & 0.0010\end{array}$

$\begin{array}{llllll}0.0009 & 0.6159 & 0.0104 & 0.0577 & 0.0009\end{array}$

$\begin{array}{lllll}0.0006 & 0.3414 & 0.0076 & 0.0535 & 0.0012\end{array}$

$\begin{array}{llllll}0.0009 & 0.5458 & 0.0109 & 0.0563 & 0.0011\end{array}$

$\begin{array}{lllll}0.0009 & 0.5206 & 0.0159 & 0.0554 & 0.0017 \\ 0.0010 & 0.5136 & 0.0207 & 0.0552 & 0.0022\end{array}$
Ages (Ma)

\% concord. \% concord.

$\underline{(206 / 238} \quad \underline{(206 / 238}$ Best Age

207/235) 207/206) (Ma) $\pm 2 s$

$\begin{array}{llll}-1.5 & -10.6 & 401.1 & 9.3\end{array}$

$\begin{array}{llll}7.4 & 42.2 & 275.1 & 6.5\end{array}$

3.7

0.7

3.8

5.2

1.3

2.9

1.4

0.6

3.2

1.5

$-0.7$

6.2

1.9

1.5

4.9

4.8

1.3

0.4

0.5

$-1.0$

$-0.5$

$-0.1$

1.2

2.1

7.2

1.4

0.5

0.2

2.1

$-0.5$

$-0.3$

1.4

2.2

0.9

0.2

$-0.1$
25.4

4.5

8.6

25.9

7.9

21.3

15.5

7.7

27.8

15.6

$-1.2$

8.8

25.4

$295.0 \quad 7.5$

$\begin{array}{ll}436.3 \quad 10.2 \\ 1664.6 & 28.0\end{array}$

$1664.6 \quad 28.0$

$422.2 \quad 10.3$

$435.0 \quad 10.2$

$292.3 \quad 7.3$

$199.7 \quad 5.9$

$208.9 \quad 5.1$

$221.0 \quad 6.2$

$211.5 \quad 5.2$

$2675.7 \quad 34.1$

$699.6 \quad 18.4$

$431.3 \quad 11.2$

$430.9 \quad 10.3$

$398.5 \quad 11.5$

$\begin{array}{lll}37.1 & 216.8 & 6.4\end{array}$

$\begin{array}{lll}5.5 & 639.4 & 15.1\end{array}$

$\begin{array}{lll}2.7 & 427.8 & 9.7\end{array}$

$\begin{array}{lll}5.4 & 240.5 & 5.8\end{array}$

$\begin{array}{lll}-6.5 & 454.6 & 10.6\end{array}$

$\begin{array}{lll}-2.6 & 476.5 & 11.0\end{array}$

$\begin{array}{lll}-0.6 & 263.6 & 6.7\end{array}$

$\begin{array}{lll}10.0 & 291.8 & 7.1\end{array}$

$\begin{array}{lll}15.8 & 296.0 & 7.0\end{array}$

$\begin{array}{lll}13.1 & 2369.7 & 28.9\end{array}$

$\begin{array}{lll}2.6 & 2472.1 & 24.0\end{array}$

$\begin{array}{lll}1.3 & 1444.9 & 23.1\end{array}$

$\begin{array}{lll}1.7 & 433.9 & 10.0\end{array}$

$\begin{array}{lll}19.7 & 226.9 & 5.6\end{array}$

$\begin{array}{lll}-2.9 & 455.5 & 10.6\end{array}$

$\begin{array}{lll}-1.6 & 468.0 & 10.9\end{array}$

$\begin{array}{lll}7.4 & 480.6 & 11.1\end{array}$

$\begin{array}{lll}16.9 & 291.7 & 7.0\end{array}$

$\begin{array}{lll}5.4 & 438.3 & 10.4\end{array}$

$\begin{array}{lll}1.1 & 424.9 & 11.0\end{array}$

$\begin{array}{lll}-0.4 & 421.2 & 12.0\end{array}$ 
Ratios

$\mathbf{U}$
Ages (Ma)

Grain Pb (ppm) Atomic

No. (ppm) Th/U

$\begin{array}{lllll}\mathrm{G} 23 & 11.7 & 335.7 & 0.36 & 0.0338\end{array}$

$\begin{array}{llll}\mathrm{G} 24 & 48.5 & 1188.9 & 0.60\end{array}$

$\begin{array}{llll}\mathrm{G} 25 & 131.3 & 1453.9 & 0.98\end{array}$

$\begin{array}{llll}\mathrm{G} 26 & 23.6 & 330.8 & 0.10\end{array}$

$\begin{array}{llll}\mathrm{G} 27 & 12.7 & 263.7 & 0.65\end{array}$

$\begin{array}{cccc}\mathrm{G} 28 & 39.6 & 1001.5 & 0.56 \\ \mathrm{G} 29 & 88.2 & 800.1 & 0.40\end{array}$

$\begin{array}{llll}\mathrm{G} 30 & 68.2 & 113.3 & 1.07\end{array}$

G31 $\quad 188.8 \quad 2316.9 \quad 0.2$

$\begin{array}{llll}\mathrm{G} 32 & 49.4 & 1299.4 & 0.51\end{array}$

$\begin{array}{llll}\mathrm{G} 33 & 61.0 & 730.8 & 0.89\end{array}$

G34 $\quad 181.6 \quad 2733.0 \quad 0.32$

$\begin{array}{llll}\text { G35 } & 93.8 & 994.5 & 0.65\end{array}$

$\begin{array}{llll}\text { G36 } & 90.4 & 301.4 & 0.87\end{array}$

G37 $48.1 \quad 318.2 \quad 0.81$

$\begin{array}{llll}\mathrm{G} 38 & 33.4 & 698.6 & 0.40\end{array}$

$\begin{array}{llll}\text { G39 } & 11.4 & 164.3 & 0.58\end{array}$

$\begin{array}{llll}\text { G40 } & 57.8 & 770.7 & 0.55\end{array}$

$\begin{array}{llll}\text { G41 } & 27.5 & 225.2 & 0.42\end{array}$

$\begin{array}{llll}\text { G42 } & 111.0 & 1197.3 & 0.59\end{array}$

$\begin{array}{llll}\text { G43 } & 10.4 & 206.3 & 0.53\end{array}$

$\begin{array}{llll}\text { G44 } & 161.0 & 758.8 & 0.37\end{array}$

$\begin{array}{llll}\mathrm{G} 45 & 49.4 & 602.1 & 0.40\end{array}$

$\begin{array}{llll}\text { G46 } & 8.0 & 62.9 & 0.30\end{array}$

G47 $22.9 \quad 476.3 \quad 0.37$

$\begin{array}{llll}\mathrm{G} 48 & 9.9 & 114.7 & 0.65\end{array}$

$\begin{array}{llll}\text { G49 } & 75.1 & 1016.2 & 0.46\end{array}$

$\begin{array}{llll}\mathrm{G} 50 & 24.9 \quad 193.0 & 0.92\end{array}$

$\begin{array}{llll}\text { G51 } & 82.9 & 451.8 & 0.73\end{array}$

$\begin{array}{llll}\text { G52 } & 325.9 & 4033.1 & 0.45\end{array}$

$\begin{array}{llll}\mathrm{G} 53 & 62.6 & 816.8 & 0.12\end{array}$

$\begin{array}{llll}\text { G54 } & 120.4 & 2284.8 & 0.63\end{array}$

$\begin{array}{llll}\text { G55 } & 72.4 & 1449.7 & 0.50\end{array}$

$\begin{array}{llll}\text { G56 } & 30.9 & 497.9 & 0.98\end{array}$

$\begin{array}{llll}\text { G57 } & 49.2 & 930.8 & 0.20\end{array}$

$\begin{array}{llll}\text { G58 } & 56.6 & 816.8 & 0.45\end{array}$

$\begin{array}{llll}G 59 & 49.2 & 614.7 & 0.80\end{array}$

$\begin{array}{llll}\text { G60 } & 22.0 & 264.4 & 0.81\end{array}$

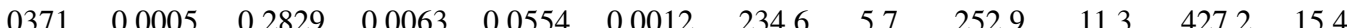

$\begin{array}{llllllllllll}0.0741 & 0.0009 & 0.5774 & 0.0091 & 0.0566 & 0.0008 & 460.6 & 10.6 & 462.8 & 14.1 & 474.4 & 11.3\end{array}$

$\begin{array}{llllllllllll}0.0756 & 0.0009 & 0.5973 & 0.0132 & 0.0573 & 0.0013 & 469.6 & 11.3 & 475.5 & 19.1 & 504.6 & 17.3\end{array}$

$\begin{array}{llllllllllll}0.0430 & 0.0006 & 0.3060 & 0.0095 & 0.0517 & 0.0016 & 271.2 & 7.0 & 271.1 & 16.0 & 270.4 & 14.7\end{array}$

$\begin{array}{llllllllllll}0.0365 & 0.0005 & 0.2589 & 0.0053 & 0.0515 & 0.0010 & 231.0 & 5.6 & 233.8 & 9.7 & 262.4 & 9.2\end{array}$

0.1056

$\begin{array}{llllll}0.4713 & 0.0057 & 10.9410 & 0.1688 & 0.1684 & 0.0024\end{array}$

$\begin{array}{lllllll}0.0831 & 0.0010 & 0.6614 & 0.0099 & 0.0577 & 0.0008\end{array}$

0.0353

$\begin{array}{llllll}0.0004 & 0.2694 & 0.0063 & 0.0553 & 0.0013\end{array}$

0.0657

0.0843

$\begin{array}{lllll}0.0009 & 0.5895 & 0.0127 & 0.0615 & 0.0013\end{array}$

$\begin{array}{lllll}0.0008 & 0.5047 & 0.0077 & 0.0557 & 0.0008 \\ 0.0010 & 0.6756 & 0.0112 & 0.0581 & 0.0009\end{array}$

$\begin{array}{lllll}0.0031 & 3.3151 & 0.0614 & 0.0957 & 0.0017\end{array}$

0.1288

0.0461

$\begin{array}{lllll}0.0016 & 1.1467 & 0.0254 & 0.0646 & 0.0014\end{array}$

$\begin{array}{lllll}0.0006 & 0.3437 & 0.0075 & 0.0541 & 0.0012\end{array}$

0.1164

$\begin{array}{lllll}0.0008 & 0.5327 & 0.0103 & 0.0560 & 0.0011\end{array}$

$\begin{array}{lllll}0.0015 & 1.0357 & 0.0230 & 0.0646 & 0.0014\end{array}$

0.0846

0.0467

$\begin{array}{lllll}0.0010 & 0.6735 & 0.0121 & 0.0578 & 0.0010\end{array}$

0.2027

$\begin{array}{lllll}0.0007 & 0.3502 & 0.0138 & 0.0545 & 0.0022 \\ 0.0024 & 2.3410 & 0.0366 & 0.0838 & 0.0012\end{array}$

$\begin{array}{lllll}0.0010 & 0.6190 & 0.0115 & 0.0570 & 0.0010\end{array}$

$\begin{array}{lllllll}0.1241 & 0.0017 & 1.2169 & 0.0375 & 0.0711 & 0.0022\end{array}$

0.0466

0.0769

$\begin{array}{lllll}0.0006 & 0.3776 & 0.0097 & 0.0588 & 0.0015\end{array}$

0.0697

$\begin{array}{lllll}0.0012 & 0.6188 & 0.0276 & 0.0584 & 0.0026\end{array}$

0.1070

$\begin{array}{lllll}0.0008 & 0.5249 & 0.0092 & 0.0546 & 0.0009 \\ 0.0015 & 0.9364 & 0.0307 & 0.0635 & 0.0021\end{array}$

$\begin{array}{llllll}0.1594 & 0.0020 & 1.5714 & 0.0302 & 0.0715 & 0.0013\end{array}$

$\begin{array}{llllll}0.0764 & 0.0009 & 0.6186 & 0.0094 & 0.0588 & 0.0008\end{array}$

0.0803

$\begin{array}{lllll}0.0010 & 0.6477 & 0.0131 & 0.0585 & 0.0012\end{array}$

0.0474

0.0467

$\begin{array}{lllll}0.0006 & 0.3435 & 0.0057 & 0.0526 & 0.0008\end{array}$

0.0505

$\begin{array}{lllll}0.0006 & 0.3365 & 0.0060 & 0.0523 & 0.0009 \\ 0.0007 & 0.3753 & 0.0098 & 0.0539 & 0.0014\end{array}$

$\begin{array}{lllll}0.0007 & 0.3753 & 0.0098 & 0.0539 & 0.0014\end{array}$

0.0655

$\begin{array}{llllll}0.0008 & 0.4937 & 0.0098 & 0.0547 & 0.0011\end{array}$

$\begin{array}{lllll}0.0009 & 0.5340 & 0.0124 & 0.0563 & 0.0013\end{array}$

0.0711

$\begin{array}{lllll}0.0010 & 0.5876 & 0.0170 & 0.0600 & 0.0017\end{array}$

$\begin{array}{llllll}646.9 & 14.8 & 663.3 & 20.1 & 720.3 & 17.3\end{array}$

$\begin{array}{llllll}4489.2 & 50.3 & 2518.1 & 35.2 & 2542.0 & 27.0\end{array}$

$\begin{array}{llllll}514.7 & 11.7 & 515.5 & 14.7 & 519.5 & 11.3\end{array}$

$\begin{array}{llllll}223.8 & 5.5 & 242.2 & 11.3 & 424.8 & 15.9\end{array}$

$\begin{array}{llllll}433.3 & 10.5 & 470.6 & 18.5 & 657.5 & 20.5\end{array}$

$\begin{array}{llllll}410.3 & 9.4 & 414.9 & 12.6 & 440.8 & 10.1\end{array}$

$\begin{array}{llllll}521.9 & 12.0 & 524.1 & 16.3 & 534.3 & 13.2\end{array}$

$\begin{array}{llllll}444.8 & 32.3 & 1484.6 & 34.1 & 1542.5 & 28.5\end{array}$

$\begin{array}{llllll}781.0 & 18.5 & 775.7 & 27.3 & 761.0 & 23.4\end{array}$

$\begin{array}{llllll}290.5 & 7.0 & 300.0 & 12.8 & 375.2 & 13.3\end{array}$

$\begin{array}{llllll}394.3 & 10.7 & 402.6 & 24.7 & 450.8 & 25.1\end{array}$

$\begin{array}{llllll}430.0 & 10.1 & 433.6 & 15.8 & 453.2 & 13.7\end{array}$

$\begin{array}{llllll}709.7 & 17.0 & 721.8 & 26.1 & 760.0 & 23.4\end{array}$

$\begin{array}{llllll}523.6 & 12.1 & 522.9 & 17.2 & 520.3 & 14.1\end{array}$

$\begin{array}{llllll}294.0 & 8.4 & 304.9 & 22.3 & 389.7 & 25.7\end{array}$

$\begin{array}{llllll}190.0 & 25.9 & 1224.8 & 26.9 & 1287.2 & 21.4\end{array}$

$\begin{array}{llllll}488.8 & 11.4 & 489.2 & 16.8 & 491.5 & 13.9\end{array}$

$\begin{array}{llllll}754.3 & 19.8 & 808.4 & 38.0 & 960.9 & 38.6\end{array}$

$\begin{array}{llllll}293.7 & 7.4 & 325.3 & 15.9 & 559.0 & 21.9\end{array}$

$\begin{array}{llllll}477.5 & 14.5 & 489.1 & 37.2 & 544.4 & 38.1\end{array}$

$\begin{array}{llllll}434.2 & 10.1 & 428.4 & 14.4 & 397.5 & 11.0\end{array}$

$\begin{array}{llllll}655.1 & 17.5 & 671.0 & 35.3 & 725.4 & 34.3\end{array}$

$\begin{array}{llllll}953.5 & 21.8 & 959.0 & 27.7 & 972.0 & 23.3\end{array}$

$\begin{array}{llllll}474.5 & 10.8 & 489.0 & 14.3 & 557.8 & 12.1\end{array}$

$\begin{array}{llllll}498.1 & 11.8 & 507.1 & 18.5 & 548.5 & 16.7\end{array}$

$\begin{array}{llllll}298.3 & 6.9 & 299.8 & 10.2 & 312.0 & 8.4\end{array}$

$\begin{array}{llllll}293.9 & 6.9 & 294.5 & 10.6 & 299.9 & 8.8\end{array}$

$\begin{array}{llllll}317.5 & 8.0 & 323.6 & 16.1 & 368.5 & 16.0\end{array}$

$\begin{array}{llllll}339.9 & 8.1 & 350.7 & 12.9 & 424.0 & 12.4\end{array}$

$\begin{array}{llllll}408.9 & 9.7 & 407.4 & 15.3 & 400.0 & 12.7\end{array}$

$\begin{array}{llllll}428.7 & 10.5 & 434.5 & 18.5 & 465.8 & 17.1\end{array}$

$\%$ concord. \% concord.

$\underline{(206 / 238} \quad \underline{(206 / 238}$ Best Age

207/235) 207/206) (Ma) $\pm 2 s$

$\begin{array}{llll}0.1 & 0.6 & 444.9 & 10.3\end{array}$

$\begin{array}{llll}6.2 & 43.1 & 214.3 & 6.1\end{array}$

7.3

0.5

1.2

$-0.1$

1.2

2.5

1.1

0.1

7.6

7.9

1.1
0.4

2.7

$-0.7$

3.2

2.1

0.8

1.7

$-0.1$

3.6

2.8

0.1
6.7

9.7

2.4

$-1.4$

2.4

0.6

3.0
1.8

1.8

0.5

0.2

1.9

3.1
-0.4

-0.4
1.3

5.7

$\begin{array}{lll}45.1 & 234.6 & 5.7\end{array}$

$\begin{array}{lll}2.9 & 460.6 & 10.6\end{array}$

$\begin{array}{lll}6.9 & 469.6 & 11.3\end{array}$

$\begin{array}{lll}-0.3 & 271.2 & 7.0\end{array}$

$\begin{array}{lll}12.0 & 231.0 & 5.6\end{array}$

$\begin{array}{lll}10.2 & 646.9 & 14.8\end{array}$

$\begin{array}{lll}2.1 & 2542.0 & 27.0\end{array}$

$\begin{array}{lll}0.9 & 514.7 & 11.7\end{array}$

$\begin{array}{lll}47.3 & 223.8 & 5.5\end{array}$

$\begin{array}{lll}34.1 & 433.3 & 10.5\end{array}$

$\begin{array}{lll}6.9 & 410.3 & 9.4\end{array}$

$\begin{array}{lll}2.3 & 521.9 & 12.0\end{array}$

$\begin{array}{lll}6.3 & 1542.5 & 28.5\end{array}$

$\begin{array}{lll}-2.6 & 781.0 & 18.5\end{array}$

$\begin{array}{lll}22.6 & 290.5 & 7.0\end{array}$

$\begin{array}{lll}12.5 & 394.3 & 10.7\end{array}$

$\begin{array}{lll}5.1 & 430.0 & 10.1\end{array}$

$\begin{array}{lll}6.6 & 709.7 & 17.0\end{array}$

$\begin{array}{lll}-0.6 & 523.6 & 12.1\end{array}$

$\begin{array}{lll}24.6 & 294.0 & 8.4\end{array}$

$\begin{array}{lll}7.6 & 1287.2 & 21.4\end{array}$

$\begin{array}{lll}0.5 & 488.8 & 11.4\end{array}$

$\begin{array}{lll}21.5 & 754.3 & 19.8\end{array}$

$\begin{array}{lll}47.5 & 293.7 & 7.4\end{array}$

$\begin{array}{lll}12.3 & 477.5 & 14.5\end{array}$

$\begin{array}{lll}-9.2 & 434.2 & 10.1\end{array}$

$\begin{array}{lll}9.7 & 655.1 & 17.5\end{array}$

$\begin{array}{lll}1.9 & 972.0 & 23.3\end{array}$

$\begin{array}{lll}14.9 & 474.5 & 10.8\end{array}$

$9.2 \quad 498.1 \quad 11.8$

$\begin{array}{lll}4.4 & 298.3 & 6.9\end{array}$

$\begin{array}{lll}2.0 & 293.9 & 6.9\end{array}$

$\begin{array}{lll}13.9 & 317.5 & 8.0\end{array}$

$\begin{array}{lll}19.8 & 339.9 & 8.1\end{array}$

$\begin{array}{lll}-2.2 & 408.9 & 9.7\end{array}$

$\begin{array}{lll}8.0 & 428.7 & 10.5\end{array}$ 
Table S1

Ratios

$\mathbf{U}$
Grain Pb (ppm) Atomic

$\mathbf{T h} / \mathrm{U}$

$\begin{array}{lccc}\text { No. } & \text { (ppm) } & & \text { Th/U } \\ \text { G61 } & 30.4 & 611.9 & 0.75\end{array}$

$\begin{array}{llll}\text { G62 } & 10.0 & 90.9 & 0.13\end{array}$

$\begin{array}{llll}\text { G63 } & 12.2 & 88.8 & 0.71\end{array}$$$
\text { G }
$$

$\begin{array}{cccc}\text { G65 } & 26.4 & 307.0 & 0.69\end{array}$

$\begin{array}{llll}\text { G66 } & 27.8 & 118.2 & 0.45\end{array}$

$\begin{array}{llll}\text { G67 } & 170.0 & 2712.8 & 0.27 \\ \text { G68 } & 318.9 & 3065.2 & 1.41\end{array}$

$\begin{array}{llll}\text { G68 } & 318.9 & 3065.2 & 1.41\end{array}$

$\begin{array}{cccc}\text { G69 } & 9.3 & 228.7 & 0.84 \\ \text { G70 } & 36.2 & 445.5 & 0.85\end{array}$

$\begin{array}{llll}\text { G71 } & 17.1 & 163.6 & 0.28\end{array}$

$\begin{array}{llll}\mathrm{G} 72 & 27.7 & 349.7 & 0.59\end{array}$

$\begin{array}{llll}\text { G73 } & 66.1 & 346.9 & 1.29 \\ \text { G74 } & 13.2 & 379.0 & 0.56\end{array}$

$\begin{array}{llll}\text { G74 } & 13.2 & 379.0 & 0.56\end{array}$

$\begin{array}{cccc}\text { G75 } & 42.7 & 577.0 & 0.48 \\ \text { G76 } & 103.6 & 1291.7 & 0.50\end{array}$

G77 858.0 \#\#\#\#\# 1.05

G78 $274.7 \quad 3240.8 \quad 0.59$

$\begin{array}{llll}\text { G79 } & 101.8 & 1437.9 & 0.58\end{array}$

$\begin{array}{llll}\text { G80 } & 48.1 & 646.9 & 0.57\end{array}$

$\begin{array}{llll}\text { G81 } & 35.7 & 499.3 & 0.51 \\ \text { G82 } & 36.1 & 203.5 & 1.39\end{array}$

$\begin{array}{llll}\text { G83 } & 64.0 & 911.9 & 0.53\end{array}$

$\begin{array}{llll}\text { G84 } & 112.7 & 2384.1 & 0.52\end{array}$

$\begin{array}{llll}\text { G85 } & 173.9 & 1270.7 & 0.28\end{array}$

$\begin{array}{llll}\text { G86 } & 29.3 & 219.6 & 0.36\end{array}$

$\begin{array}{llll}\text { G87 } & 67.7 & 482.5 & 0.44\end{array}$

G88 $\quad 47.1 \quad 985.4 \quad 0.32$

G89 $\quad 38.1 \quad 223.8 \quad 0.60$

$\begin{array}{llll}\text { G90 } & 13.6 & 164.3 & 0.60\end{array}$

G91 $\quad 77.2 \quad 2064.5 \quad 0.51$

$\begin{array}{llll}\text { G92 } & 77.3 & 611.9 & 0.49\end{array}$

$\begin{array}{llll}\text { G93 } & 48.4 & 1575.6 & 0.03\end{array}$

$\begin{array}{llll}\text { G94 } & 48.5 & 590.2 & 0.67\end{array}$

$\begin{array}{llll}\text { G95 } & 52.1 & 765.1 & 0.09\end{array}$

$\begin{array}{llll}\text { G96 } & 175.5 & 2179.2 & 0.42\end{array}$

$\begin{array}{llll}\text { G97 } & 13.1 & 383.9 & 0.36\end{array}$

G98 $\quad 151.5 \quad 2052.6 \quad 0.53$

G99 $\quad 123.4 \quad 1610.6 \quad 0.02$

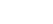

\pm s.e. $207 / 235 \pm$ s.e. $207 / 206 \pm$ s.e.

$\begin{array}{llllll}0.1145 & 0.0017 & 1.0814 & 0.0410 & 0.0685 & 0.0026\end{array}$

$\begin{array}{llllll}0.1195 & 0.0011 & 1.2104 & 0.0246 & 0.0735 & 0.0024\end{array}$

$\begin{array}{llllll}0.0462 & 0.0017 & 0.3396 & 0.0379 & 0.0533 & 0.0023\end{array}$

$\begin{array}{lllllll}0.0762 & 0.0006 & 0.5895 & 0.0324 & 0.0562 & 0.0065\end{array}$

0.2161

0.0630

$\begin{array}{lllllll}0.0759 & 0.0008 & 0.5978 & 0.0144 & 0.0572 & 0.0017\end{array}$

$\begin{array}{llllll}0.0348 & 0.0006 & 0.2538 & 0.0189 & 0.0529 & 0.0038\end{array}$

$\begin{array}{lllllll}0.0705 & 0.0006 & 0.5593 & 0.0136 & 0.0575 & 0.0029\end{array}$

0.1042

0.0317

.0686

0.0651

0.0765

0.0649

0.0662

0.1322

0.0456

0.1357

0.1284

0.1330

0.1537

0.0750

0.1166

0.0334

0.0738

0.0719

0.0771

0.0683

$\begin{array}{llllll}0.0830 & 0.0030 & 0.6640 & 0.0541 & 0.0581 & 0.0015\end{array}$
Ages (Ma)

$\%$ concord. \% concord.

$\underline{(206 / 238} \quad \underline{(206 / 238}$ Best Age

207/235) 207/206) (Ma) $\pm 2 \mathrm{~s}$

$\begin{array}{cccc}2.1 & 17.1 & 266.5 & 6.7 \\ 6.2 & 21.1 & 698.5 & 20.0\end{array}$

$9.7-29.2-727.5 \quad 12.7$

$\begin{array}{llll}9.7 & 29.2 & 727.5 & 12.7 \\ 1.9 & 15.0 & 291.2 & 20.8\end{array}$

$\begin{array}{llll}-0.5 & -3.1 & 473.1 & 7.2\end{array}$

$\begin{array}{llll}1.7 & 4.6 & 1321.9 & 44.4\end{array}$

$\begin{array}{llll}1.6 & 10.2 & 393.5 & 35.4\end{array}$

$\begin{array}{llll}0.9 & 5.3 & 471.4 & 9.3\end{array}$

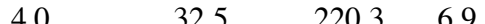

$\begin{array}{llll}4.0 & 32.5 & 220.3 & 6.9 \\ 2.6 & 14.1 & 439.4 & 7.0\end{array}$

$\begin{array}{llll}1.8 & 7.7 & 638.9 & 11.0\end{array}$

$\begin{array}{llll}-0.2 & -1.1 & 446.3 & 17.3\end{array}$

$\begin{array}{llll}0.0 & 0.0 & 900.5 & 21.6\end{array}$

$\begin{array}{llll}4.0 & 34.2 & 201.1 & 23.4\end{array}$

$427.8 \quad 5.2$

$\begin{array}{llll}3.2 & 16.1 & 468.5 & 7.2\end{array}$

$\begin{array}{llll}1.1 & 7.1 & 406.8 & 11.1\end{array}$

$\begin{array}{llll}1.2 & 6.6 & 475.0 & 10.2\end{array}$

$\begin{array}{llll}2.6 & 14.8 & 405.2 & 11.1\end{array}$

$\begin{array}{llll}2.5 & 14.2 & 423.9 & 8.2\end{array}$

$\begin{array}{llll}2.3 & 13.5 & 413.3 & 10.4\end{array}$

$\begin{array}{llll}5.6 & 18.0 & 800.1 & 5.7\end{array}$

$\begin{array}{llll}3.9 & 21.0 & 403.2 & 20.7\end{array}$

$\begin{array}{llll}0.2 & 2.2 & 287.4 & 12.6\end{array}$

$\begin{array}{llll}7.7 & 22.8 & 820.5 & 25.2\end{array}$

$\begin{array}{llll}3.2 & 11.3 & 778.8 & 9.5\end{array}$

$\begin{array}{llll}3.4 & 14.6 & 804.7 & 4.7\end{array}$

$\begin{array}{llll}1.2 & 9.7 & 296.9 & 20.6\end{array}$

$\begin{array}{llll}0.5 & 1.7 & 937.4 & 40.2 \\ 3.6 & 18.1 & 466.4 & 23.1\end{array}$

$\begin{array}{llll}3.6 & 18.1 & 466.4 & 23.1\end{array}$

$\begin{array}{llll}3.4 & 28.5 & 220.8 & 13.6\end{array}$

$\begin{array}{llll}3.1 & 11.7 & 711.1 & 5.0\end{array}$

$\begin{array}{llll}1.4 & 14.7 & 211.5 & 18.0\end{array}$

$\begin{array}{llll}6.4 & 28.3 & 458.8 & 5.0\end{array}$

$\begin{array}{llll}2.5 & 13.6 & 447.3 & 11.3\end{array}$

$\begin{array}{llll}2.3 & 12.2 & 478.9 & 10.7\end{array}$

$\begin{array}{llll}0.5 & 6.4 & 210.6 & 24.5\end{array}$

$\begin{array}{llll}1.7 & 10.0 & 425.8 & 5.4\end{array}$

$\begin{array}{llll}0.6 & 3.5 & 513.8 & 36.1\end{array}$ 
Table S1

Ratios

$\mathbf{U}$

Grain Pb (ppm) Atomic

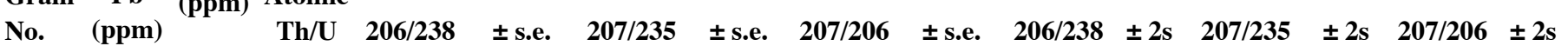

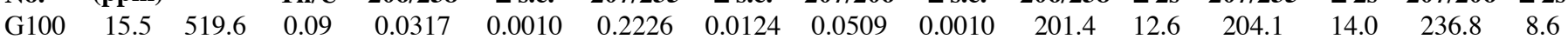

$\begin{array}{llllllllllllllll}\text { G101 } & 44.5 & 564.4 & 0.56 & 0.0722 & 0.0004 & 0.5597 & 0.0062 & 0.0562 & 0.0014 & 449.4 & 4.9 & 451.3 & 18.9 & 461.5 & 18.7\end{array}$

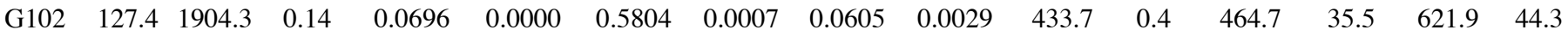

$\begin{array}{llllllllllllllll}\mathrm{G} 103 & 35.6 & 509.1 & 0.45 & 0.0663 & 0.0009 & 0.5002 & 0.0122 & 0.0548 & 0.0012 & 413.7 & 10.4 & 411.8 & 17.7 & 402.0 & 15.0\end{array}$

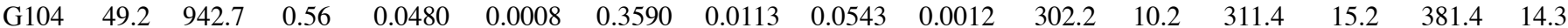

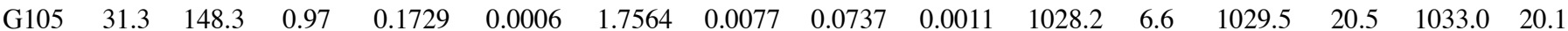

\section{Sample 13062403 - Downstream Hotan River}

\begin{tabular}{|c|c|c|c|c|c|c|c|c|c|c|c|c|c|c|}
\hline G1 & 81.4 & 927.4 & 0.96 & 0.0719 & 0.0009 & 0.5451 & 0.0109 & 0.0550 & 0.0011 & 447.8 & 10.7 & 441.8 & 16.4 & 411.0 \\
\hline G2 & 28.9 & 710.1 & 0.77 & 0.0337 & 0.0004 & 0.2318 & 0.0065 & 0.0498 & 0.0014 & 213.9 & 5.5 & 211.7 & 11.8 & 187.5 \\
\hline G3 & 134.5 & 1521.5 & 1.26 & 0.0677 & 0.0008 & 0.5026 & 0.0091 & 0.0539 & 0.0009 & 422.3 & 9.9 & 413.4 & 14.4 & 365.2 \\
\hline G4 & 39.8 & 1237.0 & 0.05 & 0.0348 & 0.0004 & 0.2372 & 0.0053 & 0.0494 & 0.0011 & 220.7 & 5.5 & 216.1 & 9.9 & 166.9 \\
\hline G5 & 12.0 & 232.8 & 0.56 & 0.0474 & 0.0007 & 0.3661 & 0.0148 & 0.0561 & 0.0023 & 298.5 & 8.7 & 316.8 & 23.7 & 454.3 \\
\hline G6 & 50.2 & 586.0 & 0.94 & 0.0700 & 0.0009 & 0.5364 & 0.0119 & 0.0556 & 0.0012 & 436.0 & 10.6 & 436.0 & 17.8 & 437.2 \\
\hline G7 & 70.9 & 943.6 & 0.48 & 0.0703 & 0.0009 & 0.5486 & 0.0108 & 0.0567 & 0.0011 & 437.7 & 10.5 & 444.1 & 16.4 & 478.3 \\
\hline G8 & 49.3 & 570.5 & 0.57 & 0.0779 & 0.0010 & 0.6084 & 0.0132 & 0.0567 & 0.0012 & 483.5 & 11.6 & 482.6 & 18.9 & 479.1 \\
\hline G9 & 45.8 & 561.6 & 0.84 & 0.0686 & 0.0009 & 0.5639 & 0.0151 & 0.0596 & 0.0016 & 427.7 & 10.9 & 454.0 & 21.8 & 590.5 \\
\hline G10 & 142.0 & 1479.3 & 1.32 & 0.0722 & 0.0009 & 0.5587 & 0.0099 & 0.0561 & 0.0010 & 449.6 & 10.6 & 450.7 & 15.2 & 456.7 \\
\hline G11 & 18.6 & 472.2 & 0.72 & 0.0342 & 0.0005 & 0.2393 & 0.0077 & 0.0508 & 0.0016 & 216.8 & 5.7 & 217.8 & 13.7 & 229.5 \\
\hline $\mathrm{G} 12$ & 8.2 & 132.3 & 0.84 & 0.0532 & 0.0010 & 0.3927 & 0.0235 & 0.0535 & 0.0033 & 334.3 & 11.6 & 336.3 & 36.4 & 350.9 \\
\hline G13 & 31.6 & 374.6 & 0.92 & 0.0701 & 0.0009 & 0.5489 & 0.0141 & 0.0568 & 0.0015 & 436.7 & 11.0 & 444.3 & 20.6 & 484.2 \\
\hline G14 & 31.9 & 223.2 & 2.55 & 0.0839 & 0.0011 & 0.6798 & 0.0195 & 0.0588 & 0.0017 & 519.2 & 13.3 & 526.6 & 25.9 & 559.7 \\
\hline G15 & 90.7 & 1227.4 & 0.37 & 0.0716 & 0.0009 & 0.5522 & 0.0103 & 0.0559 & 0.0010 & 446.0 & 10.5 & 446.5 & 15.6 & 449.6 \\
\hline G16 & 164.4 & 2624.0 & 0.45 & 0.0599 & 0.0007 & 0.4457 & 0.0076 & 540 & 0.0009 & 374.8 & 8.8 & 374.2 & 12.6 & 371.5 \\
\hline G17 & 4.4 & 63.5 & 0.55 & 0.0642 & 0.0014 & 0.4833 & 0.0400 & 0.0546 & 0.0046 & 401.3 & 17.3 & 400.4 & 57.8 & 395.9 \\
\hline G18 & 192.8 & 337.7 & 1.19 & 0.4365 & 0.0056 & 9.8535 & 0.1733 & 0.1638 & 0.0028 & 2334.7 & 49.8 & 2421.2 & 39.0 & 2495.3 \\
\hline G19 & 34.3 & 417.5 & 1.00 & 0.0668 & 0.0009 & 0.5152 & 0.0130 & 0.0560 & 0.0014 & 416.7 & 10.4 & 422.0 & 19.4 & 451.2 \\
\hline G20 & 582.5 & 2454.0 & 0.16 & 0.2401 & 0.0029 & 2.9441 & 0.0450 & 0.0890 & 0.0013 & 1387.1 & 29.8 & 1393.3 & 28.2 & 1403.5 \\
\hline G21 & 80.2 & 264.5 & 0.28 & 0.2928 & 0.0036 & 4.2874 & 0.0737 & 0.1062 & 0.0017 & 1655.5 & 36.0 & 1691.0 & 33.8 & 1735.9 \\
\hline G22 & 41.4 & 839.4 & 0.56 & 0.0452 & 0.0006 & 0.3283 & 0.0077 & 0.0527 & 0.0012 & 284.8 & 7.0 & 288.3 & 13.2 & 317.2 \\
\hline G23 & 38.2 & 123.4 & 0.16 & 0.3083 & 0.0041 & 4.6133 & 0.0983 & 0.1086 & 0.0023 & 1732.5 & 40.0 & 1751.7 & 41.4 & 1775.2 \\
\hline G24 & 25.1 & 509.1 & 0.46 & 0.0466 & 0.0006 & 0.3406 & 0.0093 & 0.0531 & 0.0014 & 293.4 & 7.5 & 297.6 & 15.5 & 332.2 \\
\hline G25 & 45.6 & 264.5 & 0.49 & 0.1600 & 0.0020 & 1.5897 & 0.0334 & 0.0721 & 0.0015 & 956.9 & 22.5 & 966.2 & 30.1 & 988.2 \\
\hline G26 & 36.3 & 184.7 & 1.12 & 0.1540 & 0.0020 & 1.5040 & 0.0368 & 0.0709 & 0.0017 & 923.2 & 22.5 & 932.0 & 33.6 & 953.4 \\
\hline G27 & 99.5 & 3246.9 & 0.08 & 0.0328 & 0.0004 & 0.2281 & 0.0041 & 0.0504 & 0.0009 & 208.2 & 5.0 & 208.6 & 7.9 & 214.9 \\
\hline G28 & 60.5 & 368.0 & 1.26 & 0.1242 & 0.0016 & 1.1114 & 0.0226 & 0.0649 & 0.0013 & 754.9 & 17.8 & 758.9 & 25.0 & 771.1 \\
\hline G29 & 22.6 & 445.6 & 0.23 & 0.0529 & 0.0007 & 0.4338 & 0.0110 & 0.0595 & 0.0015 & 332.2 & 8.3 & 365.9 & 17.4 & 586.2 \\
\hline G30 & 43.0 & 1264.3 & 0.08 & 0.0362 & 0.0005 & 0.2524 & 0.0056 & 0.0505 & 0.0011 & 229.5 & 5.6 & 228.5 & 10.2 & 219.9 \\
\hline G31 & 18.9 & 305.9 & 0.76 & 0.0536 & 0.0007 & 0.3774 & 0.0122 & 0.0511 & 0.0017 & 336.4 & 8.9 & 325.1 & 19.5 & 245.8 \\
\hline G32 & 28.5 & 140.4 & 2.47 & 0.1198 & 0.0017 & 1.0357 & 0.0351 & 0.0627 & 0.0021 & 729.3 & 19.7 & 721.8 & 38.2 & 699.1 \\
\hline
\end{tabular}

$\%$ concord. \% concord.

$\underline{(206 / 238} \quad \underline{(206 / 238}$ Best Age

207/235) 207/206) (Ma) $\pm 2 s$

$\begin{array}{llll}1.3 & 14.9 & 201.4 & 12.6\end{array}$

$\begin{array}{llll}0.4 & 2.6 & 449.4 & 4.9\end{array}$

$\begin{array}{llll}6.7 & 30.3 & 433.7 & 0.4\end{array}$

$\begin{array}{llll}-0.5 & -2.9 & 413.7 & 10.4\end{array}$

$\begin{array}{llll}3.0 & 20.8 & 302.2 & 10.2\end{array}$

$\begin{array}{llll}0.1 & 0.5 & 1033.0 & 20.1\end{array}$

$\begin{array}{llll}-1.4 & -9.0 & 447.83 & 10.70\end{array}$

$-1.1$

$\begin{array}{llll}-1.1 & -14.1 & 213.91 & 5.49 \\ -2.1 & -15.6 & 422.28 & 9.90\end{array}$

$\begin{array}{llll}-2.1 & -32.3 & 220.71 & 5.48\end{array}$

$\begin{array}{llll}5.8 & 34.3 & 298.54 & 8.74\end{array}$

$\begin{array}{llll}0.0 & 0.3 & 435.97 & 10.60\end{array}$

$\begin{array}{llll}1.4 & 8.5 & 437.66 & 10.48\end{array}$

$\begin{array}{llll}-0.2 & -0.9 & 483.46 & 11.60\end{array}$

$\begin{array}{llll}5.8 & 27.6 & 427.66 & 10.86\end{array}$

$\begin{array}{llll}0.2 & 1.6 & 449.64 & 10.58\end{array}$

$\begin{array}{llll}0.5 & 5.5 & 216.84 & 5.73\end{array}$

$\begin{array}{llll}0.6 & 4.7 & 334.32 & 11.63\end{array}$

$\begin{array}{llll}1.7 & 9.8 & 436.70 & 10.96\end{array}$

$\begin{array}{llll}1.4 & 7.2 & 519.24 & 13.32\end{array}$

$\begin{array}{llll}0.1 & 0.8 & 446.03 & 10.47\end{array}$

$\begin{array}{llll}-0.2 & -0.9 & 374.83 & 8.76\end{array}$

$\begin{array}{llll}-0.2 & -1.4 & 401.30 & 17.32\end{array}$

$\begin{array}{llll}3.6 & 6.4 & 2495.27 & 31.40\end{array}$

$\begin{array}{llll}1.2 & 7.6 & 416.73 & 10.39\end{array}$

$\begin{array}{llll}0.4 & 1.2 & 1403.51 & 21.71\end{array}$

$\begin{array}{llll}2.1 & 4.6 & 1735.87 & 27.24\end{array}$

$\begin{array}{llll}1.2 & 10.2 & 284.80 & 7.03\end{array}$

$\begin{array}{llll}1.1 & 2.4 & 1775.22 & 35.23\end{array}$

$\begin{array}{llll}1.4 & 11.7 & 293.37 & 7.51\end{array}$

$\begin{array}{llll}1.0 & 3.2 & 988.20 & 25.94\end{array}$

$\begin{array}{llll}0.9 & 3.2 & 953.38 & 30.02\end{array}$

$\begin{array}{llll}0.2 & 3.1 & 208.17 & 4.99\end{array}$

$\begin{array}{llll}0.5 & 2.1 & 754.92 & 17.78\end{array}$

$\begin{array}{llll}9.2 & 43.3 & 332.18 & 8.33\end{array}$

$\begin{array}{llll}-0.4 & -4.4 & 229.48 & 5.60\end{array}$

$\begin{array}{llll}-3.5 & -36.9 & 336.40 & 8.93\end{array}$

$\begin{array}{lll}-4.3 & 729.30 & 19.69\end{array}$ 
Ratios

$\mathbf{U}$

Grain $\mathrm{Pb}$ (ppm) Atomic

G33 $31.261 .6 \quad 0.03$

$\begin{array}{cccc}\text { G33 } & 31.2 & 861.6 & 0.03 \\ \text { G34 } & 177.3 & 709.4 & 0.67\end{array}$

$\begin{array}{lllll}\mathrm{G} 35 & 286.5 & 672.4 & 0.55 & 0.3782\end{array}$

$\begin{array}{llllll}177.3 & 709.4 & 0.67 & 0.2190 & 0.0 \\ 17.7 & 211.3 & 0.47 & 0.0790 & 0.0\end{array}$

$\begin{array}{lllll}\mathrm{G} 37 & 40.2 & 498.8 & 0.56 & 0.0737\end{array}$

$\begin{array}{llll}\text { G33 } & 151.2 & 1660.4 & 0.09\end{array}$

$\begin{array}{llll}\mathrm{G} 39 & 19.7 & 181.8 & 0.99\end{array}$

$\begin{array}{llll}\mathrm{G} 40 & 19.8 & 253.5 & 0.78\end{array}$

$\begin{array}{llllll}\mathrm{G} 41 & 195.3 & 719.0 & 0.41 & 0.2547\end{array}$

$\begin{array}{lllll}\text { G43 } & 15.1 & 160.3 & 1.00 & 0.0767\end{array}$

$\begin{array}{lllll}\mathrm{G} 44 & 26.1 & 844.6 & 0.09 & 0.0330\end{array}$

$\begin{array}{llll}\text { G45 } & 347.8 & 4969.3 & 0.13\end{array}$

G46 $23.0 \quad 269.0 \quad 1.05$

G47 $\quad 6.1 \quad 53.2 \quad 0.76$

$\begin{array}{llll}53.2 & 0.76 & 0.0991\end{array}$

\begin{tabular}{lllll} 
& 41.9 & 563.8 & 0.49 & 0.0693 \\
\hline 49 & 13.1 & 264.5 & 0.57 & 0.0453
\end{tabular}

$\begin{array}{lllll}\mathrm{G} 50 & 64.6 & 816.5 & 0.60 & 0.0716\end{array}$

$\begin{array}{lllll}\text { G51 } & 41.2 & 573.4 & 0.51 & 0.0668\end{array}$

$\begin{array}{lllll}\text { G52 } & 69.2 & 597.1 & 0.64 & 0.1037\end{array}$

$\begin{array}{llll}\mathrm{G} 53 & 5.5 & 2295.9 & 0.68\end{array}$

$\begin{array}{llll}\text { G55 } & 91.5 & 1315.3 & 0.42\end{array}$

$\begin{array}{llll}\text { G56 } & 58.3 & 769.2 & 0.47\end{array}$

$\begin{array}{llll}\text { G57 } & 142.3 & 359.1 & 0.49\end{array}$

$\begin{array}{llll}\text { G58 } & 81.7 & 1349.3 & 1.19\end{array}$

$\begin{array}{llll}\text { G59 } & 40.7 & 843.1 & 0.38\end{array}$

$\begin{array}{llll}\text { G60 } & 128.8 & 982.0 & 0.67\end{array}$

$\begin{array}{llll}\text { G61 } & 44.2 & 538.7 & 0.82\end{array}$

$\begin{array}{llll}\text { G62 } & 9.3 & 267.5 & 0.53\end{array}$

$\begin{array}{llll}\text { G63 } & 37.3 & 150.7 & 0.57\end{array}$

$\begin{array}{llll}\text { G64 } & 23.3 & 664.3 & 0.41\end{array}$

$\begin{array}{llll}\text { G65 } & 38.3 & 495.8 & 0.71\end{array}$

$\begin{array}{llll}\text { G66 } & 18.0 & 263.8 & 0.42\end{array}$

G67 $38.1 \quad 728.6 \quad 0.76$

$\begin{array}{llll}\text { G68 } & 13.3 & 359.1 & 0.61\end{array}$

G69 $\quad 144.6 \quad 1152.7 \quad 0.15$

$\begin{array}{lllll}\mathrm{G} 70 & 8.9 & 108.6 & 0.89\end{array}$

$\begin{array}{llll}\mathrm{G} 71 & 6.8 & 189.2 & 0.65\end{array}$
0.0022

0.0665

0.0710

0.3558

0.0469

0.0468
0.1152

0.0701

0.0336

0.0680

0.0653

0.0451

0.0336
0.1296

0.0688

0.0321
0.0321
0.2229
Ages (Ma)

$\%$ concord. \% concord.

$\underline{(206 / 238} \quad \underline{(206 / 238}$ Best Age

207/235) 207/206) (Ma) $\pm 2 s$

$\begin{array}{llll}-4.7 & -94.0 & 248.49 & 6.45\end{array}$

$\begin{array}{llll}0.5 & 1.3 & 1293.48 & 23.15\end{array}$

$\begin{array}{llll}0.4 & 0.7 & 2083.43 & 27.45\end{array}$

$\begin{array}{llll}0.8 & 4.7 & 490.27 & 12.79\end{array}$

$\begin{array}{llll}-0.2 & -1.2 & 458.65 & 11.17\end{array}$

$584.45 \quad 13.54$

1.6

1.1

$-0.5$

0.1

0.4

$-0.3$

$-1.1$

5.2

$-1.0$

0.3

2.4

0.1

$-0.2$

5.5

2.3

2.0

$-0.9$

$-0.2$

$-0.4$

0.0

3.4

0.2

$-2.6$

$-2.8$

2.3

0.0

0.1

2.0

2.3

2.4

$-4.3$

0.5
$545.53 \quad 14.22$

$\begin{array}{lll}418.96 & 10.99\end{array}$

$1502.28 \quad 24.10$

$1522.91 \quad 22.81$

$476.28 \quad 13.41$

$209.48 \quad 5.24$

\begin{tabular}{ll}
$456.49 \quad 10.57$ \\
\hline
\end{tabular}

$428.44 \quad 11.10$

$609.25 \quad 20.29$

$432.06 \quad 10.61$

$285.79 \quad 7.89$

$445.91 \quad 10.71$

$\begin{array}{ll}416.73 & 10.27\end{array}$

$636.23 \quad 14.95$

$13.84 \quad 0.51$

$415.10 \quad 11.12$

$414.07 \quad 9.91$

$441.94 \quad 10.59$

$295.46 \quad 7.14$

$294.66 \quad 7.27$

$703.11 \quad 16.30$

$436.64 \quad 10.72$

$203.68 \quad 6.00$

$1201.17 \quad 40.41$

$212.79 \quad 5.49$

$423.79 \quad 10.50$

$407.84 \quad 10.77$

$284.18 \quad 7.77$

$213.04 \quad 5.99$

$785.81 \quad 18.03$

$428.80 \quad 13.63$

$203.43 \quad 6.62$
$1956.37 \quad 27.90$ 
Table S1

Ratios

$\mathbf{U}$
Ages (Ma)

Grain Pb (ppm) Atomic

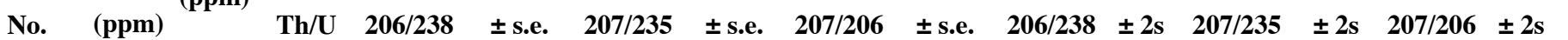

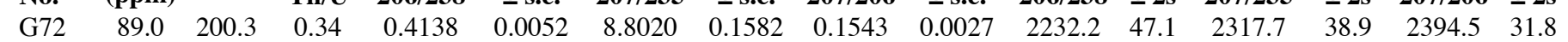
$\begin{array}{llllllllllllllll}\text { G73 } & 14.7 & 98.3 & 0.99 & 0.1216 & 0.0019 & 1.0868 & 0.0439 & 0.0648 & 0.0027 & 739.8 & 21.5 & 746.9 & 46.3 & 769.1 & 44.3\end{array}$ $\begin{array}{llllllllllllllll}\text { G74 } & 11.3 & 135.2 & 0.81 & 0.0712 & 0.0011 & 0.5510 & 0.0241 & 0.0562 & 0.0025 & 443.3 & 13.1 & 445.6 & 33.9 & 458.3 & 32.7\end{array}$ $\begin{array}{llllllllllllllll}\mathrm{G} 75 & 36.2 & 206.9 & 1.20 & 0.1353 & 0.0018 & 1.2489 & 0.0336 & 0.0670 & 0.0018 & 818.0 & 20.4 & 822.9 & 33.8 & 836.8 & 30.6\end{array}$ $\begin{array}{lllllllllllllllll}\text { G76 } & 48.2 & 780.3 & 0.08 & 0.0657 & 0.0008 & 0.5069 & 0.0118 & 0.0560 & 0.0013 & 410.1 & 10.0 & 416.3 & 18.0 & 452.0 & 16.8\end{array}$ $\begin{array}{llllllllllllllll}\text { G77 } & 51.3 & 580.1 & 0.46 & 0.0845 & 0.0011 & 0.7681 & 0.0198 & 0.0659 & 0.0017 & 523.2 & 13.1 & 578.6 & 25.4 & 803.8 & 28.5\end{array}$ $\begin{array}{llllllllllllllll}\mathrm{G} 78 & 22.4 & 62.8 & 0.97 & 0.2882 & 0.0041 & 4.1708 & 0.1154 & 0.1050 & 0.0029 & 1632.7 & 41.3 & 1668.3 & 51.3 & 1714.1 & 46.0\end{array}$ $\begin{array}{llllllllllllllll}\text { G79 } & 80.4 & 226.1 & 0.90 & 0.2916 & 0.0038 & 4.0124 & 0.0869 & 0.0998 & 0.0021 & 1649.5 & 37.6 & 1636.7 & 40.6 & 1620.9 & 34.5\end{array}$ $\begin{array}{llllllllllllllll}\text { G80 } & 51.6 & 1789.7 & 0.05 & 0.0311 & 0.0004 & 0.2134 & 0.0052 & 0.0498 & 0.0012 & 197.4 & 4.9 & 196.4 & 9.8 & 184.3 & 8.2\end{array}$ $\begin{array}{llllllllllllllll}\mathrm{G} 81 & 12.7 & 317.0 & 0.96 & 0.0331 & 0.0005 & 0.2321 & 0.0106 & 0.0509 & 0.0024 & 209.7 & 6.2 & 211.9 & 18.7 & 237.2 & 19.6\end{array}$ $\begin{array}{llll}\text { G82 } & 37.2 & 772.9 & 0.40\end{array}$ $\begin{array}{llll}\text { G83 } & 191.7 & 2106.7 & 1.45\end{array}$ $\begin{array}{llll}\text { G84 } & 115.3 & 1386.2 & 0.38\end{array}$ $\begin{array}{llll}\text { G85 } & 7.7 & 142.6 & 1.23\end{array}$

$\begin{array}{llll}\text { G86 } & 53.9 & 632.5 & 0.86\end{array}$

$\begin{array}{llll}\text { G87 } & 111.2 & 297.1 & 0.96\end{array}$

$\begin{array}{llll}\text { G88 } & 197.6 & 545.3 & 0.26\end{array}$

$\begin{array}{llll}\text { G89 } & 53.5 & 645.1 & 0.99\end{array}$

$\begin{array}{llll}\text { G90 } & 60.5 & 627.4 & 0.31 \\ \text { G91 } & 94.7 & 328.1 & 0.65\end{array}$

$\begin{array}{llll}\text { G92 } & 66.9 & 974.7 & 0.47\end{array}$

$\begin{array}{llll}\text { G93 } & 20.4 & 147.8 & 0.80\end{array}$

$\begin{array}{llll}\text { G94 } & 84.3 & 1466.0 & 0.38\end{array}$

$\begin{array}{llll}\text { G95 } & 270.4 & 3978.4 & 0.40\end{array}$

$\begin{array}{llll}\text { G96 } & 208.8 & 2873.7 & 0.45\end{array}$

$\begin{array}{llll}\text { G97 } & 103.1 & 1408.4 & 0.56\end{array}$

$\begin{array}{llll}\text { G98 } & 175.9 & 2751.8 & 0.22\end{array}$

$\begin{array}{llll}\text { G99 } & 25.5 & 399.8 & 0.37\end{array}$

$\begin{array}{llll}\text { G100 } & 343.0 & 4585.1 & 0.41\end{array}$

$\begin{array}{llll}\text { G101 } & 486.7 \quad 6100.6 & 0.44\end{array}$

$\begin{array}{llll}\mathrm{G} 102 & 42.2 & 1332.3 & 0.07\end{array}$

$\begin{array}{llll}\mathrm{G} 103 & 20.2 & 100.5 & 0.32\end{array}$

$\begin{array}{llll}\mathrm{G} 104 & 47.8 & 589.7 & 0.80\end{array}$

$\begin{array}{llll}\text { G105 } & 135.9 & 279.3 & 1.37\end{array}$

$\begin{array}{llll}\text { G106 } & 28.8 \quad 790.7 & 0.62\end{array}$

$\begin{array}{llll}\text { G107 } & 44.6 & 1446.8 & 0.04\end{array}$

$\begin{array}{llll}\mathrm{G} 108 & 50.0 & 1348.6 & 0.32\end{array}$

G109 $\quad 162.2 \quad 1520.0 \quad 0.64$

$\begin{array}{llll}\text { G110 } & 111.6 \quad 1404.7 \quad 0.35\end{array}$ $\begin{array}{lllllll}0.0461 & 0.0006 & 0.3349 & 0.0092 & 0.0527 & 0.0014\end{array}$

$\begin{array}{llllll}0.0669 & 0.0008 & 0.5270 & 0.0112 & 0.0572 & 0.0012\end{array}$

$\begin{array}{lllllll}0.0804 & 0.0010 & 0.6348 & 0.0130 & 0.0573 & 0.0011\end{array}$

$\begin{array}{llllll}0.0412 & 0.0007 & 0.2986 & 0.0162 & 0.0526 & 0.0029\end{array}$

$\begin{array}{lllllll}0.0719 & 0.0009 & 0.5701 & 0.0135 & 0.0575 & 0.0013\end{array}$

$\begin{array}{lllllll}0.3003 & 0.0038 & 4.6037 & 0.0948 & 0.1112 & 0.0023\end{array}$

0.3488

$\begin{array}{lllll}0.0043 & 6.1540 & 0.1089 & 0.1280 & 0.0022\end{array}$

0.0945

0.2530

$\begin{array}{llllll}0.0009 & 0.5225 & 0.0140 & 0.0560 & 0.0015\end{array}$

$\begin{array}{lllll}0.0012 & 0.7973 & 0.0178 & 0.0612 & 0.0013 \\ 0.0032 & 3.2965 & 0.0645 & 0.0945 & 0.0018\end{array}$

$\begin{array}{lllllll}0.0646 & 0.0008 & 0.4905 & 0.0109 & 0.0551 & 0.0012\end{array}$

$\begin{array}{lllllll}0.1176 & 0.0017 & 1.0600 & 0.0380 & 0.0654 & 0.0024\end{array}$

0.0557

$\begin{array}{lllll}0.0007 & 0.4061 & 0.0090 & 0.0529 & 0.0012\end{array}$

$\begin{array}{lllllll}0.0652 & 0.0008 & 0.5077 & 0.0104 & 0.0565 & 0.0011\end{array}$

0.0691

$\begin{array}{llllll}0.0652 & 0.0008 & 0.4962 & 0.0097 & 0.0552 & 0.0010\end{array}$

$\begin{array}{lllllll}0.0616 & 0.0009 & 0.4977 & 0.0189 & 0.0586 & 0.0023\end{array}$

0.0714

$\begin{array}{lllll}0.0009 & 0.5125 & 0.0102 & 0.0521 & 0.0010\end{array}$

$\begin{array}{llllll}0.0760 & 0.0009 & 0.6002 & 0.0111 & 0.0573 & 0.0010\end{array}$

0.0338

0.1935

$\begin{array}{lllll}0.0004 & 0.2504 & 0.0067 & 0.0537 & 0.0014 \\ 0.0027 & 2.1738 & 0.0658 & 0.0815 & 0.0025\end{array}$

$\begin{array}{lllll}0.0027 & 2.1738 & 0.0658 & 0.0815 & 0.0025\end{array}$

0.3547

0.0327

$\begin{array}{llllll}0.0009 & 0.5299 & 0.0141 & 0.0555 & 0.0015\end{array}$

$\begin{array}{lllll}0.0045 & 6.0583 & 0.1206 & 0.1239 & 0.0024 \\ 0.0004 & 0.2395 & 0.0078 & 0.0531 & 0.0017\end{array}$

0.0335

0.0368

$\begin{array}{lllll}0.0004 & 0.2284 & 0.0062 & 0.0495 & 0.0013\end{array}$

0.0953

$\begin{array}{lllll}0.0005 & 0.2594 & 0.0069 & 0.0512 & 0.0014\end{array}$

$\begin{array}{lllll}0.0012 & 0.7900 & 0.0165 & 0.0602 & 0.0012\end{array}$

$\begin{array}{lllllll}0.0775 & 0.0010 & 0.6055 & 0.0133 & 0.0567 & 0.0012\end{array}$ $\begin{array}{lll}290.3 & 7.4 & 293.3\end{array}$

$\begin{array}{lll}498.7 & 11.8 \quad 499.1\end{array}$

$260.4 \quad 8.4 \quad 265.3$

$447.7 \quad 10.9 \quad 458.0$

$1692.7 \quad 38.0 \quad 1749.9$

$\begin{array}{lll}928.8 & 40.6 & 1997.9\end{array}$

$\begin{array}{lll}454.1 & 32.4 & 1480.2\end{array}$

$\begin{array}{lll}03.2 & 9.8 & 405.3\end{array}$

$\begin{array}{lll}716.9 & 19.8 & 733.8\end{array}$

$349.3 \quad 8.4 \quad 346.0$

$\begin{array}{lll}407.2 & 9.7 & 416.9\end{array}$

$\begin{array}{lll}430.6 & 10.3 & 431.6\end{array}$

$418.4 \quad 10.0 \quad 421.8$

$\begin{array}{lll}407.0 & 9.7 & 409.1\end{array}$

$\begin{array}{lll}385.5 & 10.9 \quad 410.1\end{array}$

$444.5 \quad 10.5 \quad 420.1$

$\begin{array}{lll}472.2 & 11.0 & 477.4\end{array}$

$\begin{array}{ccc}214.5 & 5.5 & 226.9 \\ 1140.5 & 29.5 & 1172.7\end{array}$

$\begin{array}{lll}140.5 & 29.5 & 1172.7\end{array}$

$\begin{array}{lll}431.6 & 10.9 & 431.8\end{array}$

$\begin{array}{lll}1957.1 & 42.4 & 1984.3\end{array}$

$207.6 \quad 5.5 \quad 218.0$

$212.2 \quad 5.4 \quad 208.9$

$232.8 \quad 5.8 \quad 234.2$

$\begin{array}{lll}586.6 & 13.9 & 591.2\end{array}$

$481.0 \quad 11.5 \quad 480.7$ $\begin{array}{lll}417.3 & 10.0 & 429.8\end{array}$

$\begin{array}{lll}582.3 & 14.0 & 595.3\end{array}$
$\%$ concord. \% concord.

$\underline{(206 / 238} \quad \underline{(206 / 238}$ Best Age

207/235) 207/206) (Ma) $\quad \mathbf{\pm 2}$

$\begin{array}{llll}3.7 & 6.8 & 2394.47 & 31.81\end{array}$

$\begin{array}{llll}1.0 & 3.8 & 739.77 & 21.50\end{array}$

$\begin{array}{llll}0.5 & 3.3 & 443.32 & 13.12\end{array}$

0.6

1.5

9.6

2.1

$-0.8$

$-0.5$

1.0

1.0

2.9

0.1

1.8

2.3

3.3

3.5

1.0

2.2

1.8

0.5

2.3

$-0.9$

2.3

0.2

0.8

0.5

6.0

$-5.8$

1.1

5.5

2.7

0.0

1.4

4.8

$-1.6$

0.6

0.8

$-0.1$ $\begin{array}{ll}443.32 & 13.12 \\ 818.03 & 20.44\end{array}$

$\begin{array}{lll}410.08 & 10.04\end{array}$

$523.16 \quad 13.08$

$1714.14 \quad 46.04$

$1620.92 \quad 34.55$

$197.43 \quad 4.88$

$209.67 \quad 6.24$

$290.35 \quad 7.40$

$417.33 \quad 10.03$

$498.69 \quad 11.81$

$260.39 \quad 8.42$

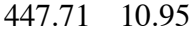

$1819.61 \quad 34.23$

$2070.82 \quad 29.95$

$422.53 \quad 10.63$

$582.27 \quad 14.02$

$1518.53 \quad 30.01$

$403.24 \quad 9.81$

$716.85 \quad 19.84$

$349.30 \quad 8.43$

$407.23 \quad 9.68$

$430.55 \quad 10.25$

$418.36 \quad 10.03$

$406.99 \quad 9.68$

$385.53 \quad 10.93$

$444.46 \quad 10.47$

$472.20 \quad 11.02$

$214.54 \quad 5.49$

$1233.29 \quad 43.46$

$431.64 \quad 10.85$

$2013.42 \quad 34.06$

$207.61 \quad 5.49$

$212.23 \quad 5.36$

$232.78 \quad 5.84$

$586.63 \quad 13.89$

$481.00 \quad 11.49$ 
Ratios

$\mathbf{U}$

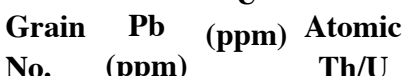

$\begin{array}{llll}\text { G111 } & 19.0 & 192.1 & 1.03\end{array}$

$\begin{array}{llll}\text { G112 } & 237.4 & 3602.3 & 0.24\end{array}$

$\begin{array}{lllll}\text { G113 } & 6.3 & 110.8 & 0.41\end{array}$

$\begin{array}{llll}\text { G114 } & 35.8 & 444.1 & 0.71 \\ \text { G115 } & 64.7 & 334.7 & 0.79\end{array}$

$\begin{array}{llll}\mathrm{G} 115 & 64.7 & 334.7 & 0.79\end{array}$

$\begin{array}{llll}\text { G116 } & 262.3 & 387.2 & 1.26\end{array}$

$\begin{array}{llll}\text { G117 } & 105.5 & 3399.8 & 0.57\end{array}$

G118 $\quad 113.0 \quad 342.9 \quad 0.71$

$\begin{array}{llll}\mathrm{G} 120 & 23.9 & 306.7 & 0.61\end{array}$

G121 $102.8 \quad 1280.6 \quad 0.63$

$\begin{array}{lllll}G 122 & 26.6 & 661.3 & 0.51\end{array}$

$\begin{array}{llll}\text { G123 } & 32.0 & 322.2 & 0.34\end{array}$

G124 $128.3 \quad 1123.2 \quad 0.29$

$\begin{array}{llll}\text { G125 } & 2.0 & 154.4 & 1.81\end{array}$

$\begin{array}{lllll}\mathrm{G} 127 & 82.8 & 914.1 & 1.04 & 0.0725\end{array}$

G128 $219.0 \quad 798.8-0.22=0$.

$\begin{array}{llll}\mathrm{G} 129 & 41.7 & 247.5 & 1.19\end{array}$

$\begin{array}{llll}G 131 & 86.0 & 273.4 & 1.18\end{array}$

$\begin{array}{lllll}\text { G132 } & 127.5 & 1827.4 & 0.28 & 0.0693\end{array}$

\begin{tabular}{|c|c|c|c|c|c|c|c|c|c|c|c|c|c|c|}
\hline G1 & 7.0 & 45.0 & 2.16 & 0.0984 & 0.0020 & 0.8159 & 0.0556 & 0.0602 & 0.0042 & 604.8 & 23.4 & 605.7 & 66.0 & 609.7 \\
\hline G2 & 21.0 & 231.6 & 0.92 & 0.0753 & 0.0010 & 0.5632 & 0.0170 & 0.0543 & 0.0016 & 467.9 & 12.0 & 453.6 & 24.2 & 381.9 \\
\hline G3 & 121.1 & 302.5 & 0.33 & 0.3746 & 0.0044 & 6.4849 & 0.0920 & 0.1256 & 0.0016 & 2050.9 & 41.6 & 2043.8 & 31.0 & 2037.3 \\
\hline G4 & 36.6 & 474.1 & 0.61 & 0.0698 & 0.0009 & 0.5404 & 0.0108 & 0.0562 & 0.0011 & 434.7 & 10.2 & 438.7 & 16.4 & 460.3 \\
\hline G5 & 370.5 & 977.4 & 0.15 & 0.3765 & 0.0044 & 7.6000 & 0.1033 & 0.1464 & 0.0018 & 2060.1 & 41.5 & 2184.9 & 30.6 & 2304.6 \\
\hline G6 & 37.9 & 219.1 & 0.42 & 0.1637 & 0.0021 & 1.6386 & 0.0356 & 0.0726 & 0.0016 & 977.1 & 22.9 & 985.2 & 31.3 & 1003.6 \\
\hline G7 & 18.8 & 319.1 & 0.80 & 0.0507 & 0.0007 & 0.3755 & 0.0097 & 0.0537 & 0.0014 & 319.0 & 8.0 & 323.7 & 15.9 & 358.5 \\
\hline G8 & 11.6 & 255.8 & 0.56 & 0.0416 & 0.0006 & 0.3090 & 0.0109 & 0.0539 & 0.0019 & 262.7 & 7.2 & 273.4 & 18.3 & 366.9 \\
\hline G9 & 7.9 & 220.8 & 0.25 & 0.0363 & 0.0005 & 0.2483 & 0.0091 & 0.0497 & 0.0018 & 229.5 & 6.2 & 225.2 & 15.9 & 180.0 \\
\hline G10 & 12.7 & 314.1 & 0.88 & 0.0339 & 0.0005 & 0.2316 & 0.0076 & 0.0495 & 0.0016 & 215.0 & 5.6 & 211.5 & 13.5 & 173.5 \\
\hline G11 & 46.0 & 248.3 & 1.31 & 0.1392 & 0.0017 & 1.3009 & 0.0248 & 0.0678 & 0.0013 & 840.2 & 19.4 & 846.1 & 25.4 & 862.1 \\
\hline G12 & 21.4 & 238.3 & 0.88 & 0.0752 & 0.0010 & 0.6334 & 0.0200 & 0.0611 & 0.0019 & 467.5 & 12.4 & 498.2 & 27.2 & 642.1 \\
\hline G13 & 5.7 & 111.7 & 0.44 & 0.0481 & 0.0007 & 0.3457 & 0.0147 & 0.0521 & 0.0023 & 302.9 & 8.7 & 301.5 & 23.8 & 291.1 \\
\hline G14 & 17.3 & 322.5 & 0.51 & 0.0498 & 0.0006 & 0.3677 & 0.0097 & 0.0536 & 0.0014 & 313.3 & 7.9 & 318.0 & 15.9 & 353.0 \\
\hline G15 & 45.1 & 285.8 & 0.88 & 0.1349 & 0.0016 & 1.2306 & 0.0227 & 0.0662 & 0.0012 & 815.9 & 18.6 & 814.6 & 24.2 & 811.8 \\
\hline G16 & 21.7 & 353.3 & 0.85 & 0.0519 & 0.0007 & 0.3893 & 0.0095 & 0.0544 & 0.0013 & 326.2 & 8.0 & 333.9 & 15.4 & 388.1 \\
\hline
\end{tabular}

Ages (Ma)

\% concord. \% concord.

$\underline{(206 / 238} \quad \underline{(206 / 238}$ Best Age

207/235) 207/206) (Ma) $\pm 2 s$

$\begin{array}{llll}-1.3 & -8.5 & 453.72 & 12.62\end{array}$

0.5

$-0.5$

0.8

$-1.1$

5.0

0.8

0.7

1.5

2.2

6.7

6.3

1.5

1.5
-1.7

2.3

4.2

0.9

2.9

2.0

5.1

1.3

0.1

$-3.2$

$-0.3$

0.9

5.7

0.8

1.5

3.9

$-1.9$

$-1.6$

0.7

6.2

$-0.5$

1.5

$-0.2$

2.3
10.8

$-3.0$

.5

$-1.9$

41.7

3.3

8.8

12.3

42.9

23.8

6.1

$-5.7$

12.7

20.6

2.1

10.3

11.4

11.7

7.8

0.8

0.8
-22.5
-0.7

5.6

10.6

2.6

11.0

28.4

$-27.5$

$-23.9$

2.5

27.2

$-4.1$

11.3

$-0.5$

15.9 $\begin{array}{ll}415.34 & 9.91\end{array}$

$341.11 \quad 11.98$

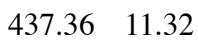
$1012.84 \quad 30.92$ $2527.60 \quad 35.47$ $180.15 \quad 4.51$ $1636.13 \quad 33.43$ $574.79 \quad 16.51$ $434.95 \quad 12.53$ $446.51 \quad 10.83$ $236.51 \quad 6.46$ $595.75 \quad 16.69$ $686.21 \quad 16.23$ $965.45 \quad 37.58$ $444.34 \quad 11.31$ $451.14 \quad 11.06$ $1572.23 \quad 32.22$ $785.98 \quad 19.86$ $448.37 \quad 11.67$ $1573.58 \quad 42.40$ $432.00 \quad 10.49$

$604.8 \quad 23.4$ $467.9 \quad 12.0$ $2037.3 \quad 23.0$

$434.7 \quad 10.2$ $2304.6 \quad 22.5$ $1003.6 \quad 27.2$

$319.0 \quad 8.0$

$262.7 \quad 7.2$

$229.5 \quad 6.2$

$215.0 \quad 5.6$

$840.2 \quad 19.4$

$467.5 \quad 12.4$

$302.9 \quad 8.7$

$313.3 \quad 7.9$

$815.9 \quad 18.6$

$326.2 \quad 8.0$ 
Table S1

Ratios

$\mathbf{U}$
Ages (Ma)

Grain Pb (ppm) Atomic

No. $($ ppm) $\quad$ Th/U $206 / 238 \quad \pm$ s.e. $\quad 207 / 235 \quad \pm$ s.e. $\quad 207 / 206 \quad \pm$ s.e. $\quad 206 / 238 \quad \pm 2 s \quad 207 / 235 \quad \pm 2 s \quad 207 / 206 \quad \pm 2 s$

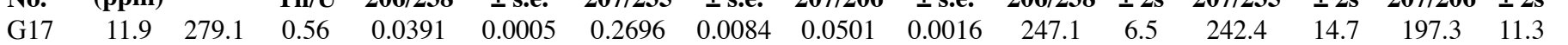

$\begin{array}{llllllllllllllll}\mathrm{G} 18 & 5.7 & 76.7 & 0.92 & 0.0623 & 0.0009 & 0.4994 & 0.0212 & 0.0581 & 0.0025 & 389.8 & 11.4 & 411.3 & 30.8 & 533.9 & 35.7\end{array}$

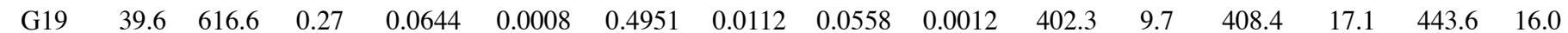

$\begin{array}{lllllllllllllllll}\mathrm{G} 20 & 13.9 & 179.2 & 0.80 & 0.0662 & 0.0009 & 0.5123 & 0.0144 & 0.0561 & 0.0016 & 413.3 & 10.4 & 420.0 & 21.2 & 457.5 & 20.6\end{array}$

$\begin{array}{llllllllllllllll}\mathrm{G} 21 & 262.9 & 680.8 & 0.72 & 0.3286 & 0.0039 & 5.2087 & 0.0717 & 0.1150 & 0.0014 & 1831.5 & 37.5 & 1854.0 & 29.3 & 1879.9 & 21.4\end{array}$

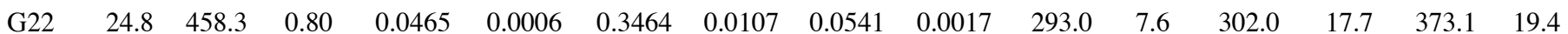

$\begin{array}{llllllllllllllll}\mathrm{G} 23 & 13.4 & 68.3 & 0.83 & 0.1655 & 0.0024 & 1.6379 & 0.0546 & 0.0718 & 0.0024 & 987.0 & 26.6 & 984.9 & 46.3 & 980.8 & 42.4\end{array}$

$\begin{array}{llllllllllllllll}\text { G24 } & 226.5 & 624.9 & 0.14 & 0.3581 & 0.0043 & 6.5565 & 0.0949 & 0.1328 & 0.0018 & 1973.0 & 40.4 & 2053.5 & 31.6 & 2135.8 & 23.9\end{array}$

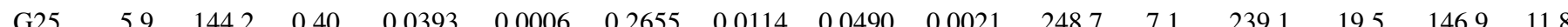

$\begin{array}{llllllllllllllll}\mathrm{G} 26 & 65.8 & 636.6 & 0.16 & 0.1064 & 0.0013 & 0.9157 & 0.0153 & 0.0625 & 0.0010 & 651.5 & 14.8 & 660.1 & 19.3 & 690.2 & 15.9\end{array}$

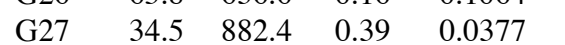

$\mathrm{G} 28 \quad 20.0 \quad 102.5 \quad 0.45 \quad 0.72$

G29 $34.7 \quad 229.1 \quad 0.56$

G30 $\quad 32.9 \quad 355.8 \quad 0.86$

G31 $\quad 54.2 \quad 331.6 \quad 1.21$

$\begin{array}{llll}\mathrm{G} 32 & 58.3 & 384.1 & 0.59\end{array}$

$\begin{array}{llll}\text { G33 } & 16.8 & 95.8 & 1.64\end{array}$

G34 $500.0 \quad 1076.6 \quad 0.44$

$\begin{array}{cccc}\mathrm{G} 35 & 41.8 & 653.3 & 0.51 \\ \mathrm{G} 36 & 193.3 & 3638.0 & 0.06\end{array}$

$\begin{array}{cccc}\text { G36 } & 193.3 & 3638.0 & 0.06 \\ \text { G37 } & 19.9 & 172.5 & 0.46\end{array}$

$\begin{array}{llll}\mathrm{G} 38 & 17.3 & 110.0 & 1.20\end{array}$

G39

G4

$$
\text { G4 }
$$$$
\text { G4 } 43
$$

G44

$$
\text { G45 }
$$

G46

$\begin{array}{llll}\text { G47 } & 90.9 & 870.8 & 0.62 \\ \text { G48 } & 34.8 & 530.8 & 0.16 \\ \text { G49 } & 42.5 & 595.0 & 0.60\end{array}$

$\begin{array}{llll}\text { G50 } & 143.3 & 400.8 & 0.2\end{array}$

G51 $33.9 \quad 370.0 \quad 1.13$

G52 $\quad 51.5 \quad 774.9 \quad 0.18$

$\begin{array}{llll}\text { G53 } & 15.3 & 290.0 & 0.62\end{array}$

G54 $\quad 30.9 \quad 615.8 \quad 1.01$

G55

$\begin{array}{lllllllllllll}0.1380 & 0.0017 & 1.2758 & 0.0266 & 0.0671 & 0.0014 & 833.2 & 19.5 & 835.0 & 27.2 & 840.3 & 23.3\end{array}$

$\begin{array}{llllllllllll}0.0775 & 0.0010 & 0.6129 & 0.0136 & 0.0574 & 0.0013 & 481.4 & 11.6 & 485.4 & 19.5 & 505.0 & 17.5\end{array}$

$\begin{array}{llllllllllll}0.1275 & 0.0016 & 1.1487 & 0.0248 & 0.0654 & 0.0014 & 773.5 & 18.2 & 776.6 & 26.8 & 785.9 & 23.3\end{array}$

$\begin{array}{llllllllllll}0.1376 & 0.0017 & 1.2853 & 0.0236 & 0.0678 & 0.0012 & 831.2 & 18.9 & 839.2 & 24.5 & 861.2 & 20.6\end{array}$

$\begin{array}{llllllllllll}0.1229 & 0.0017 & 1.0867 & 0.0338 & 0.0641 & 0.0020 & 747.3 & 19.4 & 746.9 & 36.0 & 746.3 & 33.0\end{array}$

$\begin{array}{llllllllllll}0.4238 & 0.0050 & 9.3444 & 0.1296 & 0.1600 & 0.0020 & 2277.8 & 45.2 & 2372.4 & 31.8 & 2455.1 & 23.5\end{array}$

$\begin{array}{lllllllllllll}0.0596 & 0.0007 & 0.4517 & 0.0094 & 0.0550 & 0.0011 & 373.2 & 8.9 & 378.4 & 14.9 & 411.4 & 13.7\end{array}$

0.0570

$\begin{array}{lllll}0.0007 & 0.4554 & 0.0070 & 0.0580 & 0.0008\end{array}$

$357.1 \quad 8.2 \quad 381.0$

$\begin{array}{lll}10.4 & 124.2 & 0.81 \\ 19.8 & 418.3 & 0.62\end{array}$

$\begin{array}{lllllll}0.1220 & 0.0017 & 1.0943 & 0.0359 & 0.0651 & 0.0022\end{array}$

$\begin{array}{llllll}0.0710 & 0.0011 & 0.5861 & 0.0248 & 0.0599 & 0.0026\end{array}$

$\begin{array}{llllll}664.6 & 17.0 & 682.8 & 31.7 & 743.9 & 30.5\end{array}$

$\begin{array}{llllll}742.0 & 19.8 & 750.6 & 38.1 & 776.9 & 35.9\end{array}$

$\begin{array}{llllll}442.3 & 13.0 & 468.3 & 34.2 & 598.9 & 38.8\end{array}$

$\begin{array}{lll}88.3 & 240.0 & 0.28\end{array}$

$\begin{array}{lllll}0.0006 & 0.3051 & 0.0082 & 0.0517 & 0.0014\end{array}$

$\begin{array}{lll}270.4 & 6.8 & 270.4\end{array}$

0.3512

$\begin{array}{lllll}0.0006 & 0.3381 & 0.0081 & 0.0524 & 0.0012\end{array}$

$\begin{array}{lll}294.7 & 7.3 & 295.8\end{array}$

$\begin{array}{lll}14.2 & 271.7 & 12.8\end{array}$

$\begin{array}{lll}68.7 & 281.6 & 1.10\end{array}$

0.1940

$\begin{array}{lllllllll}0.0043 & 5.4586 & 0.0945 & 0.1128 & 0.0019 & 1940.3 & 41.4 & 1894.1\end{array}$

0.0560

$\begin{array}{llllll}0.0018 & 1.3090 & 0.0295 & 0.0681 & 0.0015\end{array}$

$\begin{array}{ccc}1143.1 & 25.9 & 1141.1 \\ 842.2 & 20.0 & 849.7\end{array}$

$\begin{array}{lllll}0.0007 & 0.4104 & 0.0069 & 0.0532 & 0.0009\end{array}$

$\begin{array}{lll}351.2 & 8.2 & 349.2\end{array}$

0.0858

$\begin{array}{lllll}0.0010 & 0.6987 & 0.0127 & 0.0591 & 0.0010\end{array}$

$\begin{array}{lll}530.5 & 12.2 \quad 538.0\end{array}$

0.0678

$\begin{array}{lllll}0.0011 & 0.7682 & 0.0127 & 0.0596 & 0.0009\end{array}$

576.213 .1

0.0650

$\begin{array}{lllll}0.0008 & 0.5174 & 0.0105 & 0.0554 & 0.0011 \\ 0.0008 & 0.4977 & 0.0100 & 0.0555 & 0.0011\end{array}$

$\begin{array}{lll}422.9 & 10.0 & 423.4\end{array}$

0.0721

$\begin{array}{lllll}0.0042 & 5.9640 & 0.0915 & 0.1240 & 0.0018 \\ 0.0009 & 0.5737 & 0.0134 & 0.0578 & 0.0013\end{array}$

0.0682

$\begin{array}{llllll}0.0009 & 0.5737 & 0.0134 & 0.0578 & 0.0013\end{array}$

$\begin{array}{llll}448.6 & 10.8 & 460.4\end{array}$

$\begin{array}{lll}19.4 & 520.3 & 18.8\end{array}$

$\begin{array}{lllllll}0.0474 & 0.0006 & 0.3520 & 0.0107 & 0.0538 & 0.0016\end{array}$

$298.7 \quad 7.8$

428.7

$\begin{array}{lll}14.9 & 448.4 & 12.7\end{array}$

$\begin{array}{lll}17.6 & 364.4 & 18.7\end{array}$

$\begin{array}{lll}14.6 & 285.4 & 14.4\end{array}$

$\begin{array}{lll}14.3 & 264.2 \quad 13.8\end{array}$

$\%$ concord. \% concord.

$\underline{(206 / 238} \quad \underline{(206 / 238}$ Best Age

207/235) 207/206) (Ma) $\pm 2 s$

$\begin{array}{llll}-1.9 & -25.2 & 247.1 & 6.5\end{array}$

$\begin{array}{llll}5.2 & 27.0 & 389.8 & 11.4\end{array}$

1.5

1.6

1.2

3.0

-0.2
3.9

$-4.0$

1.3

1.2

1.0

0.2

0.8

0.4

1.0

$-0.1$

4.0

1.4

6.3

2.7

1.1

5.6

0.0

0.3

$-2.4$

$-0.2$

0.9

$-0.6$

1.4

0.4

0.1

1.0

2.1

2.6

0.8

2.5

1.1
0.7

9.3

$\begin{array}{ll}402.3 & 9.7\end{array}$

$\begin{array}{ll}413.3 & 10.4\end{array}$

$1879.9 \quad 21.4$

$293.0 \quad 7.6$

$980.8 \quad 42.4$

$\begin{array}{lll}7.6 & 2135.8 & 23.9\end{array}$

$\begin{array}{lll}-69.3 & 248.7 & 7.1\end{array}$

$\begin{array}{lll}5.6 & 651.5 & 14.8\end{array}$

$\begin{array}{lll}11.5 & 238.3 & 5.7\end{array}$

$\begin{array}{lll}2.9 & 1112.4 & 37.0\end{array}$

$\begin{array}{lll}0.8 & 833.2 & 19.5\end{array}$

$\begin{array}{lll}4.7 & 481.4 & 11.6\end{array}$

$\begin{array}{lll}1.6 & 773.5 & 18.2\end{array}$

$\begin{array}{lll}3.5 & 831.2 & 18.9\end{array}$

$\begin{array}{lll}-0.1 & 747.3 & 19.4\end{array}$

$\begin{array}{lll}7.2 & 2455.1 & 23.5\end{array}$

$\begin{array}{lll}9.3 & 373.2 & 8.9\end{array}$

$\begin{array}{lll}32.6 & 357.1 & 8.2\end{array}$

$\begin{array}{lll}10.7 & 664.6 & 17.0\end{array}$

$\begin{array}{lll}4.5 & 742.0 & 19.8\end{array}$

$\begin{array}{lll}26.1 & 442.3 & 13.0\end{array}$

$\begin{array}{lll}0.5 & 270.4 & 6.8\end{array}$

$\begin{array}{lll}3.3 & 294.7 & 7.3\end{array}$

$\begin{array}{lll}-5.2 & 1844.4 & 28.2\end{array}$

$\begin{array}{lll}-0.5 & 1137.9 & 25.6\end{array}$

$\begin{array}{lll}3.2 & 842.2 & 20.0\end{array}$

$\begin{array}{lll}-4.4 & 351.2 & 8.2\end{array}$

$\begin{array}{lll}7.0 & 530.5 & 12.2\end{array}$

$\begin{array}{lll}2.2 & 576.2 & 13.1\end{array}$

$\begin{array}{lll}0.8 & 422.9 & 10.0\end{array}$

$\begin{array}{lll}6.4 & 406.0 & 9.6\end{array}$

$\begin{array}{lll}4.2 & 2013.8 & 25.1\end{array}$

$\begin{array}{lll}13.8 & 448.6 & 10.8\end{array}$

$\begin{array}{lll}5.2 & 425.2 & 9.9\end{array}$

$\begin{array}{lll}18.0 & 298.7 & 7.8\end{array}$

$\begin{array}{lll}10.0 & 256.9 & 6.6\end{array}$

$\begin{array}{lll}6.5 & 246.9 & 6.3\end{array}$ 
Ages (Ma)

$$
\text { U }
$$

Grain $\mathrm{Pb}$ (ppm) Atomic

No. (ppm) Th/U

$\begin{array}{llll}\text { G56 } & 32.7 & 644.1 & 1.2\end{array}$

$\begin{array}{llll}\text { G57 } & 16.4 & 248.3 & 0.32 \\ \text { G58 } & 29.9 & 660.8 & 0.87\end{array}$

$\begin{array}{cccc}\text { G59 } & 84.0 & 455.0 & 1.01 \\ \text { G60 } & 113.2 & 697.4 & 0.90\end{array}$

$\begin{array}{llll}\text { G60 } & 113.2 \quad 697.4 & 0.90\end{array}$

$\begin{array}{llll}\text { G61 } & 56.9 & 735.8 & 0.51 \\ \text { G62 } & 14.6 & 188.3 & 0.53\end{array}$

$\begin{array}{cccc}\mathrm{G} 63 & 9.5 & 84.2 & 0.66\end{array}$

$\begin{array}{llll}\text { G64 } & 13.7 & 285.8 & 0.57\end{array}$

$\begin{array}{cccc}\mathrm{G} 65 & 142.4 & 799.1 & 0.33 \\ \mathrm{G} 66 & 95.9 & 599.1 & 0.59\end{array}$

$\begin{array}{llll}\text { G66 } & 95.9 & 599.1 & 0.59\end{array}$

$\begin{array}{llll}\text { G67 } & 51.8 & 132.5 & 0.98\end{array}$

$\begin{array}{cccc}\text { G68 } & 48.2 & 974.1 & 0.79 \\ \text { G69 } & 367.5 & 1787.4 & 0.50\end{array}$

$\begin{array}{llll}\text { G70 } & 27.8 & 302.5 & 1.25\end{array}$

G71 $\quad 79.6 \quad 149.2 \quad 0.52$

$\begin{array}{llll}\text { G72 } & 93.9 & 626.6 & 0.21\end{array}$

$\begin{array}{llll}\mathrm{G} 73 & 19.1 & 59.2 & 1.03\end{array}$

$\begin{array}{llll}\text { G74 } & 9.2 & 183.3 & 0.85 \\ \text { G75 } & 85.1 & 265.8 & 1.09\end{array}$

$\begin{array}{llll}\text { G75 } & 85.1 & 265.8 & 1.09\end{array}$

$\begin{array}{llll}\text { G76 } & 84.5 & 210.8 & 1.08\end{array}$

$\begin{array}{llll}\text { G77 } & 10.6 & 160.0 & 0.87 \\ \text { G78 } & 61.7 & 1689.9 & 0.62\end{array}$

$\begin{array}{llll}\text { G78 } & 61.7 & 1689.9 & 0.62\end{array}$

$\begin{array}{llll}\text { G79 } & 30.7 & 878.3 & 0.45\end{array}$

$\begin{array}{llll}\mathrm{G} 80 & 44.5 & 471.6 & 1.48\end{array}$

$\begin{array}{llll}\text { G81 } & 25.3 & 436.6 & 1.21\end{array}$

$\begin{array}{llll}\text { G82 } & 45.5 & 696.6 & 0.50\end{array}$

$\begin{array}{llll}\text { G83 } & 32.0 & 325.0 & 0.33\end{array}$

$\begin{array}{llll}\text { G84 } & 46.9 & 248.3 & 1.99\end{array}$

$\begin{array}{llll}\text { G85 } & 20.4 & 125.8 & 0.78\end{array}$

$\begin{array}{llll}\text { G86 } & 22.6 & 286.6 & 0.41\end{array}$

$\begin{array}{llll}\text { G87 } & 20.2 & 277.5 & 0.6\end{array}$

$\begin{array}{llll}\text { G88 } & 18.4 & 203.3 & 0.93\end{array}$

G89 $\quad 21.0 \quad 398.3 \quad 1.02$

$\begin{array}{llll}\text { G90 } & 19.9 & 220.8 & 0.48\end{array}$

$\begin{array}{llll}\text { G91 } & 35.1 & 770.8 & 0.71\end{array}$

G92 $34.4 \quad 415.0 \quad 1.05$

$\begin{array}{llll}\text { G93 } & 7.0 & 144.2 & 0.77\end{array}$

G94

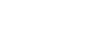

$\mathbf{2 0 6 / 2 3 8}$

$\begin{array}{lllll}0.0005 & 0.2774 & 0.0065 & 0.0518 & 0.0012\end{array}$

$\begin{array}{lllllll}0.0651 & 0.0008 & 0.4907 & 0.0132 & 0.0547 & 0.0015\end{array}$

$\begin{array}{lllllll}0.0379 & 0.0005 & 0.2709 & 0.0069 & 0.0519 & 0.0013\end{array}$

0.1498

$\begin{array}{llllll}0.1358 & 0.0017 & 1.2508 & 0.0241 & 0.0668 & 0.0013\end{array}$

$\begin{array}{llllll}0.0718 & 0.0009 & 0.5483 & 0.0102 & 0.0554 & 0.0010\end{array}$

$\begin{array}{lllllll}0.0716 & 0.0009 & 0.5332 & 0.0151 & 0.0541 & 0.0015\end{array}$

$\begin{array}{lllllll}0.1004 & 0.0015 & 0.8539 & 0.0320 & 0.0617 & 0.0023\end{array}$

0.0438

$\begin{array}{lllllll}0.1730 & 0.0021 & 1.8228 & 0.0298 & 0.0765 & 0.0012\end{array}$

0.1450

0.3129

0.0422

0.0702

0.4667

0.2582

0.0423

0.3164

0.0558

0.0332

0.0684

0.0451

0.0609

0.1231

0.1401
0.0755

0.0653

0.0707

0.0429

0.0847

0.0401
0.0666

0.0446

$\begin{array}{lllll}.0017 & 1.3950 & 0.0230 & 0.0698 & 0.0011\end{array}$

$\begin{array}{ccccccccc}.0039 & 4.7429 & 0.0870 & 0.1100 & 0.0020 & 1755.2 & 38.3 & 1774.9\end{array}$

$\begin{array}{llllllll}0.0005 & 0.3018 & 0.0060 & 0.0518 & 0.0010 & 266.6 & 6.3 & 267.8\end{array}$

$\begin{array}{lllllll}0.0022 & 2.1881 & 0.0329 & 0.0835 & 0.0012 & 1121.9 & 24.3\end{array}$

$\begin{array}{lllllllll}0.0009 & 0.5608 & 0.0138 & 0.0579 & 0.0014 & 437.6 & 10.7 & 452.0\end{array}$

$\begin{array}{llllllll}0.0056 & 10.9503 & 0.1694 & 0.1702 & 0.0025 & 2469.1 & 49.4 & 2518.9\end{array}$

$\begin{array}{llllll}0.0018 & 1.4372 & 0.0235 & 0.0689 & 0.0011\end{array}$

$\begin{array}{llllllll}0.036 & 3.4258 & 0.0883 & 0.0963 & 0.0025 & 1480.5 & 36.4 & 1510.3\end{array}$

$\begin{array}{cccccccc}0.0006 & 0.3021 & 0.0108 & 0.0519 & 0.0019 & 266.9 & 7.3 & 268.0\end{array}$

$\begin{array}{llllll}.0031 & 3.3050 & 0.0611 & 0.0946 & 0.0017\end{array}$

$\begin{array}{lllllllll}0.0039 & 4.7402 & 0.0868 & 0.1087 & 0.0019 & 1772.3 & 38.5 & 1774.4\end{array}$

$\begin{array}{lllll}0.0009 & 0.4130 & 0.0192 & 0.0537 & 0.0025\end{array}$

$\begin{array}{llllll}0.0004 & 0.2420 & 0.0074 & 0.0530 & 0.0016\end{array}$

$\begin{array}{lllll}0.0004 & 0.2389 & 0.0053 & 0.0522 & 0.0012\end{array}$

$\begin{array}{llllll}0.0008 & 0.5286 & 0.0111 & 0.0561 & 0.0012\end{array}$

$\begin{array}{lllll}0.0006 & 0.3329 & 0.0094 & 0.0536 & 0.0015\end{array}$

$\begin{array}{llllll}0.0007 & 0.4588 & 0.0093 & 0.0547 & 0.0011\end{array}$

$\begin{array}{lllll}0.0012 & 0.8780 & 0.0191 & 0.0653 & 0.0014 \\ 0.0016 & 1.0835 & 0.0306 & 0.0638 & 0.0018\end{array}$

$\begin{array}{lllll}0.0020 & 1.3046 & 0.0410 & 0.0675 & 0.0021\end{array}$

$\begin{array}{llllll}0.0010 & 0.5799 & 0.0166 & 0.0558 & 0.0016\end{array}$

$\begin{array}{llllll}0.0009 & 0.5571 & 0.0179 & 0.0619 & 0.0020\end{array}$

$\begin{array}{lllll}0.0009 & 0.5498 & 0.0162 & 0.0564 & 0.0017\end{array}$

$\begin{array}{llllll}0.0006 & 0.3152 & 0.0099 & 0.0533 & 0.0017\end{array}$

$\begin{array}{lllll}0.0011 & 0.6820 & 0.0185 & 0.0584 & 0.0016\end{array}$

$\begin{array}{lllll}0.0005 & 0.2935 & 0.0065 & 0.0531 & 0.0012\end{array}$

$\begin{array}{lllll}0.0008 & 0.5192 & 0.0117 & 0.0566 & 0.0013\end{array}$

$\begin{array}{llllll}0.0007 & 0.3297 & 0.0135 & 0.0536 & 0.0022\end{array}$

0.0699
$\%$ concord. \% concord.

$\underline{(206 / 238} \quad \underline{(206 / 238}$ Best Age

207/235) 207/206) (Ma) $\pm 2 s$

$\begin{array}{cccc}1.1 & 10.4 & 245.9 & 6.0 \\ -0.3 & -1.7 & 406.5 & 10.2\end{array}$

1.5

$\begin{array}{lccc}1.5 & 14.6 & 239.7 & 6.0 \\ 1.8 & 5.9 & 956.8 & 21.3\end{array}$

$\begin{array}{llll}0.3 & 1.3 & 821.0 & 18.8\end{array}$

$\begin{array}{llll}-0.7 & -4.0 & 446.8 & 10.5\end{array}$

$\begin{array}{llll}-2.7 & -19.4 & 445.5 & 11.2\end{array}$

$\begin{array}{llll}1.6 & 7.1 & 616.8 & 17.2\end{array}$

$\begin{array}{llll}0.3 & 2.6 & 276.6 & 7.2\end{array}$

$\begin{array}{llll}2.4 & 7.1 & 1107.1 & 20.8\end{array}$

$\begin{array}{llll}1.6 & 5.4 & 872.8 & 19.5\end{array}$

$\begin{array}{llll}1.1 & 2.4 & 1798.7 & 29.9\end{array}$

$\begin{array}{llll}0.4 & 4.2 & 266.6 & 6.3\end{array}$

$\begin{array}{llll}4.7 & 12.4 & 1281.2 & 20.4\end{array}$

$\begin{array}{llll}3.2 & 16.9 & 437.6 & 10.7\end{array}$

$\begin{array}{llll}2.0 & 3.5 & 2559.8 & 27.1\end{array}$

$\begin{array}{llll}-0.4 & -1.2 & 896.9 & 18.5\end{array}$

$\begin{array}{llll}2.0 & 4.7 & 1553.0 & 41.0\end{array}$

$\begin{array}{llll}0.4 & 4.3 & 266.9 & 7.3\end{array}$

$\begin{array}{llll}1.7 & 4.2 & 1520.1 & 28.2\end{array}$

$\begin{array}{llll}0.1 & 0.3 & 1777.2 & 29.6\end{array}$

$\begin{array}{llll}0.3 & 2.4 & 350.0 & 10.6\end{array}$

$\begin{array}{llll}4.5 & 35.9 & 210.1 & 5.5\end{array}$

$\begin{array}{llll}3.2 & 28.2 & 210.7 & 5.1\end{array}$

$\begin{array}{llll}1.1 & 6.4 & 426.3 & 10.1\end{array}$

$\begin{array}{llll}2.6 & 19.7 & 284.2 & 7.3\end{array}$

$\begin{array}{llll}0.6 & 4.3 & 381.0 & 9.0\end{array}$

$\begin{array}{llll}6.2 & 23.4 & 600.1 & 14.3\end{array}$

$\begin{array}{llll}-0.4 & -1.7 & 748.6 & 18.8\end{array}$

$\begin{array}{llll}0.3 & 1.1 & 845.4 & 22.1\end{array}$

$\begin{array}{llll}-1.0 & -5.9 & 468.9 & 11.9\end{array}$

$\begin{array}{llll}9.3 & 39.2 & 407.7 & 10.9\end{array}$

$\begin{array}{llll}1.0 & 6.0 & 440.4 & 11.2\end{array}$

$\begin{array}{llll}2.7 & 20.7 & 270.8 & 7.0\end{array}$

$\begin{array}{llll}0.7 & 3.7 & 524.4 & 13.1\end{array}$

$\begin{array}{llll}3.0 & 23.7 & 253.5 & 6.1\end{array}$

$\begin{array}{llll}2.1 & 12.5 & 415.5 & 10.0\end{array}$

$\begin{array}{llll}2.8 & 20.9 & 281.3 & 8.0\end{array}$

$\begin{array}{llll}-1.2 & -7.8 & 435.3 & 11.9\end{array}$ 
Ratios$$
\text { U }
$$

Grain Pb (ppm) Atomic

G96 $\quad 66.2 \quad 382.5 \quad 1.11$

$\begin{array}{llll}\text { G97 } & 23.5 & 296.6 & 0.68 \\ \text { G98 } & 54.3 & 366.6 & 0.57\end{array}$

$\begin{array}{llll}\text { G98 } & 54.3 & 366.6 & 0.57 \\ \text { G99 } & 18.2 & 120.0 & 0.48\end{array}$

$\begin{array}{llll}\mathrm{G} 100 & 251.2 & 750.8 & 0.18\end{array}$

G101 $\quad 188.0 \quad 1657.4 \quad 0.13$

$\begin{array}{llll}\mathrm{G} 102 & 29.1 & 555.8 & 0.39\end{array}$

$\begin{array}{llll}\mathrm{G} 103 & 123.5 & 736.6 & 0.12\end{array}$

G104 $25.4 \quad 159.2 \quad 1$.

$\begin{array}{llll}\text { G105 } & 61.7 & 825.8 & 0.40\end{array}$

$\begin{array}{llll}\text { G106 } & 182.7 & 1131.6 & 0.89\end{array}$

$\begin{array}{llll}\mathrm{G} 107 & 99.2 & 634.1 & 0.96\end{array}$

G108 $65.8 \quad 120.0 \quad 0.68$

$\begin{array}{llll}\text { G109 } & 10.6 & 164.2 & 0.3\end{array}$

$\begin{array}{llll}\text { G110 } & 85.2 & 135.8 & 1.37\end{array}$

$\begin{array}{llll}\text { G111 } & 42.5 & 897.4 & 0.49\end{array}$

$\begin{array}{llll}\text { G112 } & 40.9 & 117.5 & 0.99 \\ \text { G113 } & 31.3 & 215.0 & 0.66\end{array}$

$\begin{array}{llll}\text { G114 } & 166.6 & 872.4 & 0.89\end{array}$

$\begin{array}{llll}\text { G115 } & 21.1 & 442.5 & 0.53 \\ \text { G116 } & 85.2 & 225.0 & 0.95\end{array}$

$\begin{array}{llll}\text { G117 } & 94.8 & 654.1 & 0.15\end{array}$

$\begin{array}{llll}\text { G118 } & 18.7 & 351.6 & 1.13\end{array}$

$\begin{array}{llll}\text { G119 } & 15.7 & 135.0 & 0.71\end{array}$

$\begin{array}{llll}\text { G120 } & 22.4 \quad 505.8 \quad 0.74\end{array}$

$\begin{array}{llll}\text { G121 } & 9.9 & 195.8 & 0.87\end{array}$

$\begin{array}{llll}\text { G122 } & 10.7 & 256.6 & 0.55\end{array}$

$\begin{array}{llll}\mathrm{G} 123 & 18.6 & 92.5 & 0.82\end{array}$

$\begin{array}{llll}\mathrm{G} 124 & 41.3 & 455.0 & 0.93\end{array}$

$\begin{array}{llll}\text { G125 } & 47.6 \quad 300.0 & 0.42\end{array}$

$\begin{array}{llll}\text { G126 } & 15.4 & 262.5 & 1.09\end{array}$

$\begin{array}{llll}\text { G127 } & 23.7 & 453.3 & 0.74\end{array}$

$\begin{array}{llll}\text { G128 } & 117.4 \quad 734.9 & 0.84\end{array}$

$\begin{array}{llll}\mathrm{G} 129 & 61.4 & 95.0 & 0.89\end{array}$

Sample 13062101 - Yarkand Rive

$\begin{array}{llllllllll}\text { G1 } & 8.7 & 710.9 & 0.43 & 0.0118 & 0.0002 & 0.0806 & 0.0030 & 0.0496 & 0.0019\end{array}$

$\begin{array}{lllllllllllll}\mathrm{G} 2 & 75.1 & 2524.3 & 0.05 & 0.0320 & 0.0004 & 0.2223 & 0.0036 & 0.0505 & 0.0008 & 202.8 & 4.5 & 203.8\end{array}$
Ages (Ma)

$\%$ concord. \% concord.

$\underline{(206 / 238} \quad \underline{(206 / 238}$ Best Age

207/235) 207/206) (Ma) $\pm 2 \mathrm{~s}$

$\begin{array}{llll}3.7 & 26.9 & 270.7 & 7.4\end{array}$

$\begin{array}{llll}0.5 & 1.7 & 829.3 & 19.2\end{array}$

$\begin{array}{llll}-0.1 & -0.3 & 438.9 & 10.8\end{array}$

$\begin{array}{llll}1.3 & 4.8 & 816.2 & 19.0\end{array}$

$\begin{array}{llll}3.7 & 12.0 & 846.5 & 22.6\end{array}$

$\begin{array}{llll}1.1 & 2.3 & 1879.4 & 25.5\end{array}$

$\begin{array}{llll}9.4 & 28.6 & 725.2 & 16.5\end{array}$

$\begin{array}{llll}8.7 & 42.9 & 317.3 & 8.1\end{array}$

$\begin{array}{llll}2.5 & 7.4 & 1115.5 & 22.2\end{array}$

$\begin{array}{llll}0.9 & 3.4 & 764.8 & 18.4\end{array}$

$\begin{array}{llll}-0.9 & -6.0 & 448.6 & 10.6\end{array}$

$\begin{array}{llll}0.9 & 3.5 & 816.0 & 18.3\end{array}$

$\begin{array}{llll}4.1 & 13.8 & 780.5 & 18.2\end{array}$

$\begin{array}{llll}0.9 & 1.7 & 2494.9 & 33.3\end{array}$

$\begin{array}{llll}3.1 & 17.5 & 401.2 & 11.8\end{array}$

$\begin{array}{llll}1.1 & 2.0 & 2458.9 & 32.1\end{array}$

$\begin{array}{llll}2.9 & 21.8 & 280.2 & 6.8\end{array}$

$\begin{array}{llll}0.8 & 1.8 & 1643.7 & 33.0\end{array}$

$\begin{array}{llll}2.3 & 8.2 & 783.6 & 20.2\end{array}$

$\begin{array}{llll}1.2 & 4.0 & 987.6 & 21.4\end{array}$

$\begin{array}{llll}3.7 & 26.7 & 272.5 & 7.3\end{array}$

$\begin{array}{llll}1.3 & 2.7 & 1776.2 & 29.5\end{array}$

$\begin{array}{llll}0.6 & 2.2 & 896.4 & 20.2\end{array}$

$\begin{array}{llll}1.8 & 14.9 & 265.5 & 7.2\end{array}$

$\begin{array}{llll}4.2 & 16.7 & 621.3 & 16.6\end{array}$

$\begin{array}{llll}2.0 & 18.1 & 244.2 & 6.2\end{array}$

$\begin{array}{llll}2.8 & 21.8 & 267.8 & 7.3\end{array}$

$\begin{array}{llll}0.1 & 1.4 & 244.1 & 6.5\end{array}$

$\begin{array}{llll}0.5 & 1.6 & 1034.9 & 41.9\end{array}$

$\begin{array}{llll}4.4 & 21.1 & 465.5 & 11.6\end{array}$

$\begin{array}{llll}1.7 & 5.7 & 962.9 & 27.4\end{array}$

$\begin{array}{llll}0.6 & 5.2 & 293.7 & 7.6\end{array}$

$\begin{array}{llll}4.8 & 31.1 & 287.5 & 7.3\end{array}$

$\begin{array}{llll}0.0 & 0.0 & 824.7 & 18.8\end{array}$

$\begin{array}{llll}7.1 & 11.3 & 3231.7 & 33.6\end{array}$

$\begin{array}{llll}1.7 & 51.2 & 40.2 & 1.0\end{array}$

$\begin{array}{llll}4.0 & 57.1 & 75.5 & 2.0\end{array}$

$\begin{array}{llll}0.5 & 6.2 & 202.8 & 4.5\end{array}$ 
Ages (Ma)

$$
\text { U }
$$

Grain $\mathrm{Pb}$ (ppm) Atomic

$\mathbf{T h} / \mathbf{U}$ $\begin{array}{ccc}\text { (ppm) } & & \text { Th/U } \\ 4.0 & 116.6 & 0.40\end{array}$ $\begin{array}{lll}371.2 & 597.7 & 0.57\end{array}$

$\begin{array}{lll}10.9 & 688.8 & 0.60\end{array}$

$\begin{array}{lll}53.5 & 296.3 & 0.95 \\ 25.7 & 1542.7 & 0.44\end{array}$

$\begin{array}{lll}25.7 & 1542.7 & 0.44\end{array}$

$\begin{array}{lll}4.1 & 237.5 & 0.54\end{array}$

$\begin{array}{ccc}5.1 & 299.7 & 0.65 \\ 50.9 & 658.1 & 0.38\end{array}$

$\begin{array}{llll}\mathrm{G} 10 & 50.9 & 658.1 & 0.38\end{array}$

$\begin{array}{cccc}\text { G11 } & 11.6 & 77.5 & 1.21 \\ \text { G12 } & 35.4 & 1566.5 & 1.59\end{array}$

$\begin{array}{llll}\mathrm{G} 13 & 12.6 & 778.2 & 0.44\end{array}$

$\begin{array}{llll}\text { G14 } & 16.1 & 222.2 & 0.52\end{array}$

$\begin{array}{llll}\text { G15 } & 10.6 & 131.1 & 0.66 \\ \text { G16 } & 65.1 & 848.0 & 0.46\end{array}$

$\begin{array}{cccc}\text { G16 } & 65.1 & 848.0 & 0.46 \\ \text { G17 } & 101.4 & 1307.7 & 0.05\end{array}$

$\begin{array}{llll}\mathrm{G} 18 & 83.7 & 1042.9 & 0.80\end{array}$

$\begin{array}{llll}\text { G19 } & 31.7 & 391.6 & 0.24\end{array}$

$\begin{array}{llll}\mathrm{G} 20 & 4.7 & 369.5 & 0.7\end{array}$

$\begin{array}{llll}\text { G2 } 1 & 34.7 \quad 269.0 & 0.63\end{array}$

$\begin{array}{llll}\mathrm{G} 22 & 1.4 & 413.8 & 0.55\end{array}$

$\begin{array}{llll}\mathrm{G} 23 & 108.6 & 400.1 & 0.02\end{array}$

$\begin{array}{llll}\mathrm{G} 24 & 6.3 & 64.7 & 0.8\end{array}$

$\begin{array}{llll}\text { G25 } & 10.9 & 608.7 & 0.26\end{array}$

$\begin{array}{llll}\text { G26 } & 18.8 & 1071.9 & 0.08\end{array}$

$\begin{array}{llll}\mathrm{G} 27 & 5.0 & 269.9 & 0.72\end{array}$

$\begin{array}{llll}\text { G28 } & 26.4 & 678.5 & 0.81\end{array}$

$\begin{array}{llll}\text { G29 } & 31.7 & 762.8 & 0.68\end{array}$

$\begin{array}{llll}\mathrm{G} 30 & 34.9 & 303.1 & 0.70\end{array}$

G31 $\quad 79.6 \quad 2245.1 \quad 0.45$

$\begin{array}{llll}\mathrm{G} 32 & 33.3 & 321.8 & 1.41\end{array}$

$\begin{array}{llll}\text { G33 } & 4.3 & 252.0 & 0.62\end{array}$

$\begin{array}{llll}\text { G34 } & 96.5 & 3272.7 & 0.30\end{array}$

$\begin{array}{llll}\mathrm{G} 35 & 31.3 & 75.8 & 1.15\end{array}$

$\begin{array}{llll}\text { G36 } & 8.4 & 404.4 & 1.4\end{array}$

$\begin{array}{llll}\mathrm{G} 37 & 59.7 & 412.9 & 0.81\end{array}$

$\begin{array}{llll}\mathrm{G} 38 & 50.9 & 637.7 & 0.75\end{array}$

$\begin{array}{llll}\text { G39 } & 6.3 & 1557.2 & 0.97\end{array}$

$\begin{array}{llll}\text { G40 } & 141.6 & 3600.5 & 0.55\end{array}$

$\begin{array}{llll}\text { G41 } & 10.2 \quad 470.0 \quad 1.45\end{array}$
$206 / 238 \pm$ s.e. $207 / 235 \pm$ s.e. $207 / 206 \pm$ s.e. $206 / 238 \pm 2 s \quad 207 / 235 \quad \pm 2 s \quad 207 / 206 \quad 2 s$

$\begin{array}{llllllllllll}0.0310 & 0.0005 & 0.2121 & 0.0116 & 0.0497 & 0.0028 & 196.7 & 6.1 & 195.3 & 20.5 & 179.6 & 18.3\end{array}$

0.5567

$\begin{array}{llllll}0.1503 & 0.0017 & 1.4630 & 0.0249 & 0.0706 & 0.0012 \\ 0.0160 & 0.0002 & 0.1072 & 0.0023 & 0.0486 & 0.0011\end{array}$

$\begin{array}{llllll}0.0152 & 0.0002 & 0.1031 & 0.0045 & 0.0493 & 0.0022\end{array}$

0.0751

0.1160

0.0161

0.0675

0.0725
0.0728

0.0834

0.0696

0.0111

0.1162

0.0031

0.2840
0.0817

0.0181

0.0189
0.0162

0.0330

0.0371
0.1020

0.0336

0.0768

0.0152

0.3181

0.0154

0.1232

0.0692

0.0362

0.0157 $\begin{array}{lllll}0.0009 & 0.5920 & 0.0104 & 0.0572 & 0.0010\end{array}$

$\begin{array}{llllll}0.0015 & 1.0381 & 0.0293 & 0.0649 & 0.0019\end{array}$

$\begin{array}{llllll}0.0009 & 0.5265 & 0.0183 & 0.0566 & 0.0020\end{array}$

$\begin{array}{llllll}0.0009 & 0.5561 & 0.0171 & 0.0557 & 0.0017\end{array}$

$\begin{array}{llllll}0.0009 & 0.5638 & 0.0107 & 0.0562 & 0.0011\end{array}$

$\begin{array}{lllll}0.0010 & 0.7019 & 0.0117 & 0.0611 & 0.0010\end{array}$

$\begin{array}{llllll}0.0008 & 0.5390 & 0.0092 & 0.0562 & 0.0009\end{array}$

$\begin{array}{lllll}0.0010 & 0.6555 & 0.0131 & 0.0582 & 0.0011\end{array}$

$\begin{array}{lllll}0.0002 & 0.0804 & 0.0064 & 0.0525 & 0.0043\end{array}$

$\begin{array}{lllll}0.0015 & 1.0179 & 0.0246 & 0.0636 & 0.0015 \\ 0.0001 & 0.0208 & 0.0022 & 0.0495 & 0.0054\end{array}$

$\begin{array}{lllll}0.0033 & 4.1480 & 0.0675 & 0.1060 & 0.0017\end{array}$

$\begin{array}{llllll}0.0013 & 0.6933 & 0.0318 & 0.0616 & 0.0029\end{array}$

$\begin{array}{lllll}0.0002 & 0.1199 & 0.0037 & 0.0482 & 0.0015\end{array}$

$\begin{array}{lllll}0.0002 & 0.1288 & 0.0032 & 0.0496 & 0.0012\end{array}$

$\begin{array}{llllll}0.0003 & 0.1208 & 0.0056 & 0.0540 & 0.0026\end{array}$

$\begin{array}{lllll}0.0004 & 0.2273 & 0.0055 & 0.0500 & 0.0012\end{array}$

$\begin{array}{lllll}0.0005 & 0.2638 & 0.0059 & 0.0516 & 0.0011\end{array}$

$\begin{array}{llllll}0.0013 & 0.8509 & 0.0200 & 0.0605 & 0.0014\end{array}$

$\begin{array}{lllll}0.0004 & 0.2362 & 0.0045 & 0.0510 & 0.0010\end{array}$

$\begin{array}{lllll}0.0009 & 0.5986 & 0.0135 & 0.0565 & 0.0013\end{array}$

$\begin{array}{lllll}0.0004 & 0.2033 & 0.0037 & 0.0503 & 0.0009\end{array}$

$\begin{array}{lllll}0.0002 & 0.1014 & 0.0043 & 0.0478 & 0.0021 \\ 0.0015 & 1.1088 & 0.0220 & 0.0653 & 0.0013\end{array}$

$\begin{array}{lllll}0.0008 & 0.5351 & 0.0112 & 0.0562 & 0.0011\end{array}$

$\begin{array}{lllll}0.0004 & 0.2513 & 0.0047 & 0.0504 & 0.0009\end{array}$

$\begin{array}{lllll}0.0002 & 0.1044 & 0.0043 & 0.0481 & 0.0020\end{array}$ $\begin{array}{lllll}0.0002 & 0.1074 & 0.0023 & 0.0485 & 0.0010 \\ 0.0002 & 0.1013 & 0.0037 & 0.0474 & 0.0018\end{array}$

$\begin{array}{lllll}0.0003 & 0.1001 & 0.0064 & 0.0477 & 0.0031\end{array}$

$\begin{array}{lllll}0.0040 & 4.9672 & 0.1031 & 0.1133 & 0.0023\end{array}$

$\begin{array}{llllll}0.0001 & 0.0220 & 0.0010 & 0.0481 & 0.0023\end{array}$ $\begin{array}{llllll}93.0 & 51.3 & 3075.8 & 33.0 & 3225.2 & 25.2\end{array}$

$\begin{array}{llllll}93.0 & 2.3 & 97.2 & 6.0 & 201.0 & 10.9\end{array}$

$\begin{array}{llllll}902.6 & 19.4 & 915.3 & 24.5 & 946.7 & 20.6\end{array}$

$\begin{array}{llllll}102.5 & 2.4 & 103.4 & 4.9 & 126.2 & 5.1\end{array}$

$\begin{array}{llllll}102.3 & 3.0 & 102.6 & 10.0 & 109.6 & 10.2\end{array}$

$\begin{array}{lllllll}97.1 & 2.8 & 99.6 & 8.8 & 160.2 & 13.1\end{array}$

$\begin{array}{llllll}466.9 & 10.3 & 472.2 & 15.6 & 498.9 & 13.5\end{array}$

$\begin{array}{llllll}707.5 & 17.3 & 723.0 & 32.4 & 772.1 & 30.8\end{array}$

$\begin{array}{llllll}102.6 & 2.4 & 103.6 & 4.8 & 125.7 & 5.1\end{array}$

$\begin{array}{llllll}99.2 & 2.7 & 97.9 & 7.4 & 68.9 & 4.9\end{array}$

$\begin{array}{llllll}421.3 & 11.1 & 429.5 & 26.4 & 474.8 & 26.7\end{array}$

$\begin{array}{llllll}451.1 & 11.3 & 449.0 & 24.3 & 438.8 & 22.0\end{array}$

$\begin{array}{llllll}453.0 & 10.2 & 454.0 & 16.1 & 459.9 & 13.8\end{array}$

$\begin{array}{llllll}516.3 & 11.4 & 539.9 & 16.5 & 641.7 & 15.3\end{array}$

$\begin{array}{llllll}433.6 & 9.6 & 437.8 & 14.3 & 460.7 & 12.3\end{array}$

$\begin{array}{llllll}506.7 & 11.6 & 511.8 & 18.4 & 535.4 & 16.3\end{array}$

$\begin{array}{llllll}71.2 & 2.9 & 78.5 & 12.7 & 306.8 & 43.2\end{array}$

$\begin{array}{llllll}708.5 & 16.7 & 712.9 & 27.8 & 727.4 & 25.0\end{array}$

$\begin{array}{llllll}19.6 & 0.9 & 20.9 & 4.6 & 170.6 & 34.2\end{array}$

$\begin{array}{llllll}611.5 & 33.3 & 1663.8 & 32.0 & 1731.2 & 26.0\end{array}$

$\begin{array}{llllll}506.1 & 15.4 & 534.8 & 41.0 & 659.9 & 45.4\end{array}$

$\begin{array}{llllll}115.3 & 2.9 & 115.0 & 7.3 & 109.1 & 6.4\end{array}$

$\begin{array}{llllll}103.8 & 3.2 & 115.8 & 10.9 & 369.8 & 29.4\end{array}$

$209.1 \quad 5.0 \quad 207.9$

$\begin{array}{lll}234.7 & 5.6 & 237.7\end{array}$

$626.2 \quad 14.7 \quad 625.2$

$\begin{array}{lll}213.1 & 5.0 & 215.3\end{array}$

$\begin{array}{lll}477.1 & 11.3 & 476.3\end{array}$

$97.4 \quad 3.3$

$\begin{array}{lll}186.2 & 4.4 & 187.9\end{array}$

$\begin{array}{lll}1780.6 & 39.3 & 1813.8\end{array}$

$\begin{array}{cccccc}98.5 & 2.8 & 98.1 & 8.5 & 89.4 & 7.3\end{array}$

$\begin{array}{llllll}748.7 & 17.1 & 757.6 & 24.2 & 784.7 & 20.9\end{array}$

$\begin{array}{llllll}431.0 & 10.1 & 435.2 & 16.8 & 458.3 & 15.0\end{array}$

$\begin{array}{llllll}21.4 & 0.6 & 22.1 & 2.2 & 103.7 & 9.2\end{array}$

$\begin{array}{llllll}100.6 & 2.8 & 100.8 & 8.3 & 105.2 & 8.2\end{array}$ $\begin{array}{llllll}120.4 & 2.9 & 123.0 & 6.4 & 175.4 & 8.0\end{array}$ $\begin{array}{llllll}0.0160 & 0.0002 & 0.1064 & 0.0052 & 0.0482 & 0.0024\end{array}$
$\%$ concord. \% concord.

$\underline{(206 / 238} \quad \underline{(206 / 238}$ Best Age

207/235) 207/206) (Ma) $\pm 2 s$

$\begin{array}{llll}-0.7 & -9.5 & 196.7 & 6.1\end{array}$

$\begin{array}{llll}7.2 & 11.5 & 3225.2 & 25.2\end{array}$

$\begin{array}{llll}4.3 & 53.7 & 93.0 & 2.3\end{array}$

$\begin{array}{llll}1.4 & 4.7 & 946.7 & 20.6\end{array}$

$\begin{array}{llll}0.9 & 18.8 & 102.5 & 2.4\end{array}$

$1023 \quad 3.0$

$\begin{array}{llll}2.5 & 39.4 & 97.1 & 2.8\end{array}$

$\begin{array}{llll}1.1 & 6.4 & 466.9 & 10.3\end{array}$

$\begin{array}{llll}2.1 & 8.4 & 707.5 & 17.3\end{array}$

$\begin{array}{llll}0.9 & 18.3 & 102.6 & 2.4\end{array}$

$\begin{array}{llll}-1.2 & -43.9 & 99.2 & 2.7\end{array}$

$\begin{array}{llll}1.9 & 11.3 & 421.3 & 11.1\end{array}$

$\begin{array}{llll}-0.5 & -2.8 & 451.1 & 11.3\end{array}$

$\begin{array}{llll}0.2 & 1.5 & 453.0 & 10.2\end{array}$

$\begin{array}{llll}4.4 & 19.5 & 516.3 & 11.4\end{array}$

$\begin{array}{llll}1.0 & 5.9 & 433.6 & 9.6\end{array}$

$\begin{array}{llll}1.0 & 5.4 & 506.7 & 11.6\end{array}$

$\begin{array}{llll}9.3 & 76.8 & 71.2 & 2.9\end{array}$

$\begin{array}{llll}0.6 & 2.6 & 708.5 & 16.7\end{array}$

$\begin{array}{llll}6.0 & 88.5 & 19.6 & 0.9\end{array}$

$\begin{array}{llll}3.1 & 6.9 & 1731.2 & 26.0\end{array}$

$\begin{array}{llll}5.4 & 23.3 & 506.1 & 15.4\end{array}$

$\begin{array}{llll}-0.3 & -5.7 & 115.3 & 2.9\end{array}$

$\begin{array}{llll}2.1 & 31.3 & 120.4 & 2.9\end{array}$

$\begin{array}{llll}10.3 & 71.9 & 103.8 & 3.2\end{array}$

$\begin{array}{llll}-0.6 & -6.7 & 209.1 & 5.0\end{array}$

$\begin{array}{llll}1.3 & 12.8 & 234.7 & 5.6\end{array}$

$\begin{array}{llll}-0.2 & -0.6 & 626.2 & 14.7\end{array}$

$\begin{array}{llll}1.0 & 11.5 & 213.1 & 5.0\end{array}$

$\begin{array}{llll}-0.2 & -0.7 & 477.1 & 11.3\end{array}$

$\begin{array}{llll}-0.5 & -13.3 & 97.4 & 3.3\end{array}$

$\begin{array}{llll}0.9 & 11.0 & 186.2 & 4.4\end{array}$

$\begin{array}{llll}1.8 & 3.9 & 1852.8 & 34.6\end{array}$

$\begin{array}{llll}-0.4 & -10.2 & 98.5 & 2.8\end{array}$

$\begin{array}{llll}1.2 & 4.6 & 748.7 & 17.1\end{array}$

$\begin{array}{llll}1.0 & 6.0 & 431.0 & 10.1\end{array}$

$\begin{array}{llll}3.2 & 79.3 & 21.4 & 0.6\end{array}$

$\begin{array}{llll}-0.6 & -6.8 & 229.0 & 5.4\end{array}$

$\begin{array}{llll}0.2 & 4.3 & 100.6 & 2.8\end{array}$ 
Table S1

\section{Ratios}

Grain Pb $\begin{gathered}\text { U } \\ \text { (ppm) Atomic }\end{gathered}$

No. (ppm) $\quad$ Th/U 206/238

G42 $\quad 91.4 \quad 1164.7 \quad 0.67 \quad 0.0700$

$\begin{array}{lllll}\text { G43 } & 51.0 & 333.7 & 0.77 & 0.1311\end{array}$
+ s.e. $\quad 207 / 235$

$0.0009 \quad 0.5392$
$0.0016 \quad 1.2208$ $0.0108 \quad 0.0559$

$0.0270 \quad 0.0676$
Ages (Ma)

$\%$ concord. \% concord.

(206/238 (206/238 Best Age

207/235) 207/206)

$\begin{array}{lllllll}0.0011 & 436.1 & 10.2 & 437.9 & 16.1 & 448.0 & 13.9\end{array}$

$\begin{array}{lllllll}0.0015 & 793.9 & 18.6 & 810.2 & 27.8 & 856.0 & 25.0\end{array}$

0.4
2.0

2.7
7.3

2.7
7.3

(Ma) $\pm 2 \mathrm{~s}$

$436.1 \quad 10.2$

$793.9 \quad 18.6$ 


\begin{tabular}{|c|c|c|c|}
\hline Sample No. & 13062401 & Summary & \\
\hline Irrad. No. & GAR 60 & Central age & $9.9 \pm 2.4 \mathrm{Ma}$ \\
\hline Position & 0 & age dispersion & $180.5 \%$ \\
\hline Date & 23/04/19 & & \\
\hline Analyst & $\mathrm{AC}$ & Pooled age & $8.2 \pm 0.5 \mathrm{Ma}$ \\
\hline zeta (CN5) & 338 & Mean age & $11.6 \pm 3.3 \mathrm{Ma}$ \\
\hline zeta error & 5 & & \\
\hline No. of grains & 62 & $\begin{array}{l}\text { Chi squared } \\
\mathrm{P}(\text { chi-sq })\end{array}$ & $\begin{array}{c}1172.91 \text { with } \\
0.00 \%\end{array}$ \\
\hline Total Ns & $309.00016 \mathrm{tr}$ & & \\
\hline Total Ni & $10676 \mathrm{tr}$ & & \\
\hline Total Nd & $4640 \mathrm{tr}$ & & \\
\hline rho-d (CN5) & $1.674 \mathrm{E}+06 \mathrm{tr} / \mathrm{cm}^{2}$ & & \\
\hline mean rho-s & $2.396 \mathrm{E}+05 \mathrm{tr} / \mathrm{cm}^{2}$ & & \\
\hline mean rho-i & $4.979 \mathrm{E}+06 \mathrm{tr} / \mathrm{cm}^{2}$ & & \\
\hline mean U & $37.2 \mathrm{ppm}$ & & \\
\hline
\end{tabular}

$61 \mathrm{df}$

\begin{tabular}{|c|c|c|c|c|c|c|c|c|c|}
\hline Crystal & Ng & Ns & rho-s & $\mathbf{N i}$ & rho-i & $\mathbf{N s} / \mathbf{N i}$ & $\underset{(\mathbf{p p m})}{\mathbf{U}}$ & $\begin{array}{l}\text { Age } \\
\text { (Ma) }\end{array}$ & Error \\
\hline 1 & 60 & 3 & $3.608 \mathrm{E}+04$ & 192 & $2.309 \mathrm{E}+06$ & 0.0156 & 17.2 & 4.4 & 2.6 \\
\hline 2 & 40 & 3 & $5.411 \mathrm{E}+04$ & 202 & $3.644 \mathrm{E}+06$ & 0.0149 & 27.2 & 4.2 & 2.4 \\
\hline 3 & 21 & 1 & $3.436 \mathrm{E}+04$ & 84 & $2.886 \mathrm{E}+06$ & 0.0119 & 21.6 & 3.4 & 3.4 \\
\hline 4 & 20 & 5 & $1.804 \mathrm{E}+05$ & 280 & $1.010 \mathrm{E}+07$ & 0.0179 & 75.4 & 5.0 & 2.3 \\
\hline 5 & 14 & 1 & $5.154 \mathrm{E}+04$ & 124 & $6.390 \mathrm{E}+06$ & 0.0081 & 47.7 & 2.3 & 2.3 \\
\hline 6 & 20 & 0 & $3.608 \mathrm{E}-01$ & 54 & $1.948 \mathrm{E}+06$ & 0.0000 & 14.5 & 0.0 & 0.0 \\
\hline 7 & 15 & 2 & $9.620 \mathrm{E}+04$ & 60 & $2.886 \mathrm{E}+06$ & 0.0333 & 21.6 & 9.4 & 6.8 \\
\hline 8 & 25 & 2 & $5.772 \mathrm{E}+04$ & 114 & $3.290 \mathrm{E}+06$ & 0.0175 & 24.6 & 5.0 & 3.5 \\
\hline 9 & 12 & 5 & $3.006 \mathrm{E}+05$ & 146 & $8.778 \mathrm{E}+06$ & 0.0342 & 65.5 & 9.7 & 4.4 \\
\hline 10 & 25 & 1 & $2.886 \mathrm{E}+04$ & 150 & $4.329 \mathrm{E}+06$ & 0.0067 & 32.3 & 1.9 & 1.9 \\
\hline 11 & 40 & 2 & $3.608 \mathrm{E}+04$ & 98 & $1.768 \mathrm{E}+06$ & 0.0204 & 13.2 & 5.8 & 4.1 \\
\hline 12 & 21 & 20 & $6.871 \mathrm{E}+05$ & 354 & $1.216 \mathrm{E}+07$ & 0.0565 & 90.8 & 16.0 & 3.7 \\
\hline 13 & 20 & 3 & $1.082 \mathrm{E}+05$ & 212 & $7.648 \mathrm{E}+06$ & 0.0142 & 57.1 & 4.0 & 2.3 \\
\hline 14 & 8 & 15 & $1.353 \mathrm{E}+06$ & 43 & $3.878 \mathrm{E}+06$ & 0.3488 & 29.0 & 97.9 & 29.4 \\
\hline 15 & 60 & 2 & $2.405 E+04$ & 88 & $1.058 \mathrm{E}+06$ & 0.0227 & 7.9 & 6.4 & 4.6 \\
\hline 16 & 30 & 1 & $2.405 E+04$ & 66 & $1.587 \mathrm{E}+06$ & 0.0152 & 11.9 & 4.3 & 4.3 \\
\hline 17 & 30 & 1 & $2.405 \mathrm{E}+04$ & 43 & $1.034 \mathrm{E}+06$ & 0.0233 & 7.7 & 6.6 & 6.7 \\
\hline 18 & 30 & 9 & $2.165 \mathrm{E}+05$ & 460 & $1.106 \mathrm{E}+07$ & 0.0196 & 82.6 & 5.5 & 1.9 \\
\hline 19 & 40 & 6 & $1.082 E+05$ & 456 & $8.225 \mathrm{E}+06$ & 0.0132 & 61.4 & 3.7 & 1.5 \\
\hline 20 & 60 & 2 & $2.405 E+04$ & 138 & $1.659 \mathrm{E}+06$ & 0.0145 & 12.4 & 4.1 & 2.9 \\
\hline 21 & 15 & 2 & $9.620 \mathrm{E}+04$ & 142 & $6.830 \mathrm{E}+06$ & 0.0141 & 51.0 & 4.0 & 2.8 \\
\hline 22 & 14 & 1 & $5.154 \mathrm{E}+04$ & 15 & $7.730 \mathrm{E}+05$ & 0.0667 & 5.8 & 18.8 & 19.5 \\
\hline 23 & 15 & 4 & $1.924 \mathrm{E}+05$ & 82 & $3.944 \mathrm{E}+06$ & 0.0488 & 29.5 & 13.8 & 7.1 \\
\hline 24 & 36 & 4 & $8.017 \mathrm{E}+04$ & 318 & $6.373 E+06$ & 0.0126 & 47.6 & 3.6 & 1.8 \\
\hline 25 & 30 & 3 & $7.215 \mathrm{E}+04$ & 108 & $2.597 \mathrm{E}+06$ & 0.0278 & 19.4 & 7.9 & 4.6 \\
\hline 26 & 16 & 5 & $2.255 \mathrm{E}+05$ & 64 & $2.886 \mathrm{E}+06$ & 0.0781 & 21.6 & 22.1 & 10.3 \\
\hline 27 & 15 & 1 & $4.810 \mathrm{E}+04$ & 87 & $4.185 E+06$ & 0.0115 & 31.2 & 3.3 & 3.3 \\
\hline 28 & 15 & 1 & $4.810 \mathrm{E}+04$ & 135 & $6.494 \mathrm{E}+06$ & 0.0074 & 48.5 & 2.1 & 2.1 \\
\hline 29 & 36 & 4 & $8.017 \mathrm{E}+04$ & 296 & $5.932 \mathrm{E}+06$ & 0.0135 & 44.3 & 3.8 & 1.9 \\
\hline 30 & 40 & 1 & $1.804 \mathrm{E}+04$ & 148 & $2.670 \mathrm{E}+06$ & 0.0068 & 19.9 & 1.9 & 1.9 \\
\hline 31 & 36 & 4 & $8.017 \mathrm{E}+04$ & 216 & $4.329 \mathrm{E}+06$ & 0.0185 & 32.3 & 5.2 & 2.6 \\
\hline 32 & 27 & 2 & $5.344 \mathrm{E}+04$ & 276 & $7.375 \mathrm{E}+06$ & 0.0072 & 55.1 & 2.0 & 1.5 \\
\hline
\end{tabular}

Clift et al. 


\begin{tabular}{|c|c|c|c|c|c|c|c|c|c|}
\hline Crystal & Ng & Ns & rho-s & $\mathbf{N i}$ & rho-i & $\mathrm{Ns} / \mathrm{Ni}$ & $\underset{(\mathbf{p p m})}{\mathbf{U}}$ & $\begin{array}{c}\text { Age } \\
\text { (Ma) }\end{array}$ & Error \\
\hline 33 & 10 & 13 & $9.380 \mathrm{E}+05$ & 176 & $1.270 \mathrm{E}+07$ & 0.0739 & 94.8 & 20.9 & 6.0 \\
\hline 34 & 30 & 3 & $7.215 \mathrm{E}+04$ & 208 & $5.002 E+06$ & 0.0144 & 37.4 & 4.1 & 2.4 \\
\hline 35 & 10 & 3 & $2.165 \mathrm{E}+05$ & 84 & $6.061 E+06$ & 0.0357 & 45.3 & 10.1 & 5.9 \\
\hline 36 & 12 & 3 & $1.804 \mathrm{E}+05$ & 135 & $8.117 \mathrm{E}+06$ & 0.0222 & 60.6 & 6.3 & 3.7 \\
\hline 37 & 28 & 2 & $5.154 \mathrm{E}+04$ & 140 & $3.608 E+06$ & 0.0143 & 26.9 & 4.0 & 2.9 \\
\hline 38 & 9 & 45 & $3.608 E+06$ & 165 & $1.323 \mathrm{E}+07$ & 0.2727 & 98.8 & 76.7 & 12.9 \\
\hline 39 & 60 & 11 & $1.323 \mathrm{E}+05$ & 1320 & $1.587 \mathrm{E}+07$ & 0.0083 & 118.5 & 2.4 & 0.7 \\
\hline 40 & 40 & 0 & $1.804 \mathrm{E}-01$ & 136 & $2.453 E+06$ & 0.0000 & 18.3 & 0.0 & 0.0 \\
\hline 41 & 40 & 1 & $1.804 \mathrm{E}+04$ & 408 & $7.359 E+06$ & 0.0025 & 55.0 & 0.7 & 0.7 \\
\hline 42 & 12 & 7 & $4.209 \mathrm{E}+05$ & 103 & $6.193 E+06$ & 0.0680 & 46.2 & 19.2 & 7.5 \\
\hline 43 & 21 & 12 & $4.123 \mathrm{E}+05$ & 100 & $3.436 \mathrm{E}+06$ & 0.1200 & 25.7 & 33.9 & 10.4 \\
\hline 44 & 24 & 0 & $3.006 \mathrm{E}-01$ & 41 & $1.233 \mathrm{E}+06$ & 0.0000 & 9.2 & 0.0 & 0.0 \\
\hline 45 & 21 & 0 & $3.436 \mathrm{E}-01$ & 79 & $2.714 \mathrm{E}+06$ & 0.0000 & 20.3 & 0.0 & 0.0 \\
\hline 46 & 12 & 3 & $1.804 \mathrm{E}+05$ & 56 & $3.367 \mathrm{E}+06$ & 0.0536 & 25.1 & 15.1 & 9.0 \\
\hline 47 & 30 & 2 & $4.810 \mathrm{E}+04$ & 243 & $5.844 \mathrm{E}+06$ & 0.0082 & 43.6 & 2.3 & 1.7 \\
\hline 48 & 16 & 1 & $4.509 \mathrm{E}+04$ & 53 & $2.390 \mathrm{E}+06$ & 0.0189 & 17.8 & 5.3 & 5.4 \\
\hline 49 & 40 & 0 & $1.804 \mathrm{E}+00$ & 88 & $1.587 \mathrm{E}+06$ & 0.0000 & 11.9 & 0.0 & 0.0 \\
\hline 50 & 30 & 2 & $4.810 \mathrm{E}+04$ & 128 & $3.078 \mathrm{E}+06$ & 0.0156 & 23.0 & 4.4 & 3.1 \\
\hline 51 & 20 & 4 & $1.443 E+05$ & 256 & $9.235 \mathrm{E}+06$ & 0.0156 & 69.0 & 4.4 & 2.2 \\
\hline 52 & 30 & 3 & $7.215 \mathrm{E}+04$ & 230 & $5.532 \mathrm{E}+06$ & 0.0130 & 41.3 & 3.7 & 2.1 \\
\hline 53 & 14 & 54 & $2.783 E+06$ & 90 & $4.638 \mathrm{E}+06$ & 0.6000 & 34.6 & 167.5 & 28.9 \\
\hline 54 & 60 & 4 & $4.810 \mathrm{E}+04$ & 124 & $1.491 \mathrm{E}+06$ & 0.0323 & 11.1 & 9.1 & 4.6 \\
\hline 55 & 20 & 1 & $3.608 \mathrm{E}+04$ & 101 & $3.644 \mathrm{E}+06$ & 0.0099 & 27.2 & 2.8 & 2.8 \\
\hline 56 & 12 & 9 & $5.411 \mathrm{E}+05$ & 182 & $1.094 \mathrm{E}+07$ & 0.0495 & 81.7 & 14.0 & 4.8 \\
\hline 57 & 18 & 3 & $1.203 \mathrm{E}+05$ & 138 & $5.532 \mathrm{E}+06$ & 0.0217 & 41.3 & 6.1 & 3.6 \\
\hline 58 & 30 & 0 & $2.405 \mathrm{E}-01$ & 50 & $1.203 E+06$ & 0.0000 & 9.0 & 0.0 & 0.0 \\
\hline 59 & 100 & 1 & $7.215 E+03$ & 204 & $1.472 \mathrm{E}+06$ & 0.0049 & 11.0 & 1.4 & 1.4 \\
\hline 60 & 15 & 2 & $9.620 \mathrm{E}+04$ & 63 & $3.030 \mathrm{E}+06$ & 0.0317 & 22.6 & 9.0 & 6.4 \\
\hline 61 & 100 & 0 & $7.215 \mathrm{E}-02$ & 172 & $1.241 \mathrm{E}+06$ & 0.0000 & 9.3 & 0.0 & 0.0 \\
\hline 62 & 20 & 4 & $1.443 \mathrm{E}+05$ & 152 & $5.483 E+06$ & 0.0263 & 40.9 & 7.4 & 3.8 \\
\hline
\end{tabular}

Clift et al. 


\begin{tabular}{|c|c|c|c|}
\hline $\begin{array}{l}\text { Sample No. } \\
\text { Irrad. No. }\end{array}$ & $\begin{array}{l}\text { WC13062403 } \\
\text { GAR } 60\end{array}$ & $\begin{array}{l}\text { Central age } \\
\text { age dispersion }\end{array}$ & $\begin{array}{l}28.0 \pm 7.4 \mathrm{Ma} \\
148.4 \%\end{array}$ \\
\hline Position & 0 & & \\
\hline Date & $25 / 04 / 19$ & Pooled age & $22.4 \pm 1.3 \mathrm{Ma}$ \\
\hline Analyst & $\mathrm{AC}$ & & \\
\hline zeta (CN5) & 338 & Mean age & $37.0 \pm 11.0 \mathrm{Ma}$ \\
\hline zeta error & 5 & Chi squared & 816.82 with \\
\hline No. of grains & 33 & $P($ chi-sq $)$ & $0.00 \%$ \\
\hline Total Ns & $372.00001 \mathrm{tr}$ & & \\
\hline Total Ni & $4683 \mathrm{tr}$ & & \\
\hline Total Nd & $4640 \mathrm{tr}$ & & \\
\hline rho-d (CN5) & $1.674 \mathrm{E}+06 \mathrm{tr} / \mathrm{cm}^{2}$ & & \\
\hline mean rho-s & $7.514 \mathrm{E}+05 \mathrm{tr} / \mathrm{cm}^{2}$ & & \\
\hline mean rho-i & $6.568 \mathrm{E}+06 \mathrm{tr} / \mathrm{cm}^{2}$ & & \\
\hline mean U & $49.0 \mathrm{ppm}$ & & \\
\hline
\end{tabular}

\begin{tabular}{|c|c|c|c|c|c|c|c|c|c|}
\hline Crystal & $\mathbf{N g}$ & Ns & rho-s & $\mathbf{N i}$ & rho-i & $\mathbf{N s} / \mathbf{N i}$ & $\underset{(\mathbf{p p m})}{\mathbf{U}}$ & $\begin{array}{c}\text { Age } \\
\text { (Ma) }\end{array}$ & Error \\
\hline 1 & 8 & 1 & $9.019 \mathrm{E}+04$ & 17 & $1.533 \mathrm{E}+06$ & 0.0588 & 11.4 & 16.6 & 17.1 \\
\hline 2 & 10 & 2 & $1.443 \mathrm{E}+05$ & 49 & $3.535 \mathrm{E}+06$ & 0.0408 & 26.4 & 11.5 & 8.3 \\
\hline 3 & 18 & 1 & $4.008 \mathrm{E}+04$ & 107 & $4.289 \mathrm{E}+06$ & 0.0093 & 32.0 & 2.6 & 2.7 \\
\hline 4 & 24 & 2 & $6.013 \mathrm{E}+04$ & 167 & $5.020 \mathrm{E}+06$ & 0.0120 & 37.5 & 3.4 & 2.4 \\
\hline 5 & 20 & 1 & $3.608 E+04$ & 162 & $5.844 \mathrm{E}+06$ & 0.0062 & 43.6 & 1.7 & 1.8 \\
\hline 6 & 28 & 3 & $7.730 \mathrm{E}+04$ & 110 & $2.834 \mathrm{E}+06$ & 0.0273 & 21.2 & 7.7 & 4.5 \\
\hline 7 & 15 & 4 & $1.924 \mathrm{E}+05$ & 146 & $7.023 \mathrm{E}+06$ & 0.0274 & 52.4 & 7.7 & 3.9 \\
\hline 8 & 10 & 20 & $1.443 \mathrm{E}+06$ & 58 & $4.185 \mathrm{E}+06$ & 0.3448 & 31.2 & 96.8 & 25.1 \\
\hline 9 & 9 & 29 & $2.325 \mathrm{E}+06$ & 110 & $8.818 \mathrm{E}+06$ & 0.2636 & 65.8 & 74.2 & 15.5 \\
\hline 10 & 8 & 16 & $1.443 \mathrm{E}+06$ & 35 & $3.157 \mathrm{E}+06$ & 0.4571 & 23.6 & 128.0 & 38.7 \\
\hline 11 & 21 & 1 & $3.436 \mathrm{E}+04$ & 68 & $2.336 \mathrm{E}+06$ & 0.0147 & 17.4 & 4.2 & 4.2 \\
\hline 12 & 9 & 51 & $4.089 \mathrm{E}+06$ & 88 & $7.055 \mathrm{E}+06$ & 0.5795 & 52.7 & 161.9 & 28.6 \\
\hline 13 & 30 & 5 & $1.203 \mathrm{E}+05$ & 234 & $5.628 \mathrm{E}+06$ & 0.0214 & 42.0 & 6.0 & 2.7 \\
\hline 14 & 24 & 12 & $3.608 \mathrm{E}+05$ & 211 & $6.343 \mathrm{E}+06$ & 0.0569 & 47.4 & 16.1 & 4.8 \\
\hline 15 & 27 & 3 & $8.017 \mathrm{E}+04$ & 147 & $3.928 \mathrm{E}+06$ & 0.0204 & 29.3 & 5.8 & 3.4 \\
\hline 16 & 8 & 14 & $1.263 \mathrm{E}+06$ & 220 & $1.984 \mathrm{E}+07$ & 0.0636 & 148.2 & 18.0 & 5.0 \\
\hline 17 & 20 & 12 & $4.329 \mathrm{E}+05$ & 212 & $7.648 \mathrm{E}+06$ & 0.0566 & 57.1 & 16.0 & 4.8 \\
\hline 18 & 8 & 22 & $1.984 \mathrm{E}+06$ & 22 & $1.984 \mathrm{E}+06$ & 1.0000 & 14.8 & 276.9 & 83.6 \\
\hline 19 & 10 & 13 & $9.380 \mathrm{E}+05$ & 166 & $1.198 \mathrm{E}+07$ & 0.0783 & 89.4 & 22.1 & 6.4 \\
\hline 20 & 28 & 23 & $5.927 \mathrm{E}+05$ & 384 & $9.895 \mathrm{E}+06$ & 0.0599 & 73.9 & 16.9 & 3.6 \\
\hline 21 & 25 & 14 & $4.040 \mathrm{E}+05$ & 480 & $1.385 \mathrm{E}+07$ & 0.0292 & 103.4 & 8.2 & 2.2 \\
\hline 22 & 10 & 12 & $8.658 \mathrm{E}+05$ & 178 & $1.284 \mathrm{E}+07$ & 0.0674 & 95.9 & 19.0 & 5.7 \\
\hline 23 & 15 & 0 & $4.810 \mathrm{E}-01$ & 87 & $4.185 \mathrm{E}+06$ & 0.0000 & 31.2 & 0.0 & 0.0 \\
\hline 24 & 8 & 7 & $6.313 E+05$ & 43 & $3.878 \mathrm{E}+06$ & 0.1628 & 29.0 & 45.9 & 18.7 \\
\hline 25 & 21 & 3 & $1.031 \mathrm{E}+05$ & 169 & $5.806 \mathrm{E}+06$ & 0.0178 & 43.4 & 5.0 & 2.9 \\
\hline 26 & 16 & 1 & $4.509 \mathrm{E}+04$ & 110 & $4.960 \mathrm{E}+06$ & 0.0091 & 37.0 & 2.6 & 2.6 \\
\hline 27 & 32 & 1 & $2.255 \mathrm{E}+04$ & 128 & $2.886 \mathrm{E}+06$ & 0.0078 & 21.6 & 2.2 & 2.2 \\
\hline 28 & 8 & 6 & $5.411 \mathrm{E}+05$ & 114 & $1.028 \mathrm{E}+07$ & 0.0526 & 76.8 & 14.9 & 6.2 \\
\hline 29 & 21 & 2 & $6.871 \mathrm{E}+04$ & 181 & $6.219 \mathrm{E}+06$ & 0.0110 & 46.4 & 3.1 & 2.2 \\
\hline 30 & 12 & 11 & $6.614 \mathrm{E}+05$ & 168 & $1.010 \mathrm{E}+07$ & 0.0655 & 75.4 & 18.5 & 5.8 \\
\hline 31 & 10 & 4 & $2.886 \mathrm{E}+05$ & 82 & $5.916 \mathrm{E}+06$ & 0.0488 & 44.2 & 13.8 & 7.1 \\
\hline 32 & 10 & 74 & $5.339 \mathrm{E}+06$ & 116 & $8.369 \mathrm{E}+06$ & 0.6379 & 62.5 & 178.0 & 26.6 \\
\hline 33 & 18 & 2 & $8.017 E+04$ & 114 & $4.570 \mathrm{E}+06$ & 0.0175 & 34.1 & 5.0 & 3.5 \\
\hline
\end{tabular}

Clift et al. 


\begin{tabular}{|c|c|c|c|}
\hline Sample No. & WC13062402 & Summary & \\
\hline Irrad. No. & GAR 60 & Central age & $37.3 \pm 7.5 \mathrm{Ma}$ \\
\hline Position & 0 & age dispersion & $138.8 \%$ \\
\hline Date & 28/04/19 & & \\
\hline Analyst & $\mathrm{AC}$ & Pooled age & $29.1 \pm 1.3 \mathrm{Ma}$ \\
\hline zeta (CN5) & 338 & Mean age & $50.8 \pm 10.4 \mathrm{Ma}$ \\
\hline zeta error & 5 & & \\
\hline No. of grains & 50 & $\begin{array}{l}\text { Chi squared } \\
\mathrm{P}(\text { chi-sq) }\end{array}$ & $\begin{array}{c}1328.88 \text { with } \\
0.00 \%\end{array}$ \\
\hline Total Ns & $642.00001 \mathrm{tr}$ & & \\
\hline Total Ni & $6217 \mathrm{tr}$ & & \\
\hline Total Nd & $4640 \mathrm{tr}$ & & \\
\hline rho-d (CN5) & $1.674 \mathrm{E}+06 \mathrm{tr} / \mathrm{cm}^{2}$ & & \\
\hline mean rho-s & $5.632 \mathrm{E}+05 \mathrm{tr} / \mathrm{cm}^{2}$ & & \\
\hline mean rho-i & $4.202 \mathrm{E}+06 \mathrm{tr} / \mathrm{cm}^{2}$ & & \\
\hline mean U & $31.4 \mathrm{ppm}$ & & \\
\hline
\end{tabular}

$49 \mathrm{df}$

\begin{tabular}{|c|c|c|c|c|c|c|c|c|c|}
\hline Crystal & $\mathrm{Ng}$ & Ns & rho-s & $\mathbf{N i}$ & rho-i & $\mathbf{N s} / \mathbf{N i}$ & $\underset{(\mathbf{p p m})}{\mathbf{U}}$ & $\begin{array}{c}\text { Age } \\
\text { (Ma) }\end{array}$ & Error \\
\hline 1 & 20 & 9 & $3.247 \mathrm{E}+05$ & 29 & $1.046 \mathrm{E}+06$ & 0.3103 & 7.8 & 87.2 & 33.3 \\
\hline 2 & 16 & 1 & $4.509 \mathrm{E}+04$ & 58 & $2.615 \mathrm{E}+06$ & 0.0172 & 19.5 & 4.9 & 4.9 \\
\hline 3 & 36 & 5 & $1.002 \mathrm{E}+05$ & 95 & $1.904 \mathrm{E}+06$ & 0.0526 & 14.2 & 14.9 & 6.8 \\
\hline 4 & 36 & 6 & $1.203 \mathrm{E}+05$ & 182 & $3.648 \mathrm{E}+06$ & 0.0330 & 27.2 & 9.3 & 3.9 \\
\hline 5 & 8 & 70 & $6.313 \mathrm{E}+06$ & 94 & $8.478 \mathrm{E}+06$ & 0.7447 & 63.3 & 207.3 & 32.9 \\
\hline 6 & 40 & 3 & $5.411 \mathrm{E}+04$ & 120 & $2.165 \mathrm{E}+06$ & 0.0250 & 16.2 & 7.1 & 4.1 \\
\hline 7 & 12 & 6 & $3.608 \mathrm{E}+05$ & 144 & $8.658 \mathrm{E}+06$ & 0.0417 & 64.7 & 11.8 & 4.9 \\
\hline 8 & 24 & 2 & $6.013 \mathrm{E}+04$ & 156 & $4.690 \mathrm{E}+06$ & 0.0128 & 35.0 & 3.6 & 2.6 \\
\hline 9 & 49 & 2 & $2.945 \mathrm{E}+04$ & 226 & $3.328 \mathrm{E}+06$ & 0.0088 & 24.8 & 2.5 & 1.8 \\
\hline 10 & 10 & 9 & $6.494 \mathrm{E}+05$ & 49 & $3.535 \mathrm{E}+06$ & 0.1837 & 26.4 & 51.8 & 18.8 \\
\hline 11 & 50 & 4 & $5.772 \mathrm{E}+04$ & 144 & $2.078 \mathrm{E}+06$ & 0.0278 & 15.5 & 7.9 & 4.0 \\
\hline 12 & 24 & 1 & $3.006 \mathrm{E}+04$ & 75 & $2.255 \mathrm{E}+06$ & 0.0133 & 16.8 & 3.8 & 3.8 \\
\hline 13 & 35 & 2 & $4.123 E+04$ & 33 & $6.803 \mathrm{E}+05$ & 0.0606 & 5.1 & 17.1 & 12.5 \\
\hline 14 & 30 & 6 & $1.443 \mathrm{E}+05$ & 98 & $2.357 \mathrm{E}+06$ & 0.0612 & 17.6 & 17.3 & 7.3 \\
\hline 15 & 30 & 2 & $4.810 \mathrm{E}+04$ & 85 & $2.044 E+06$ & 0.0235 & 15.3 & 6.7 & 4.8 \\
\hline 16 & 20 & 2 & $7.215 \mathrm{E}+04$ & 82 & $2.958 \mathrm{E}+06$ & 0.0244 & 22.1 & 6.9 & 4.9 \\
\hline 17 & 16 & 16 & $7.215 \mathrm{E}+05$ & 288 & $1.299 \mathrm{E}+07$ & 0.0556 & 97.0 & 15.7 & 4.0 \\
\hline 18 & 40 & 0 & $1.804 \mathrm{E}-01$ & 168 & $3.030 \mathrm{E}+06$ & 0.0000 & 22.6 & 0.0 & 0.0 \\
\hline 19 & 20 & 4 & $1.443 \mathrm{E}+05$ & 234 & $8.442 \mathrm{E}+06$ & 0.0171 & 63.0 & 4.8 & 2.4 \\
\hline 20 & 21 & 30 & $1.031 \mathrm{E}+06$ & 190 & $6.528 \mathrm{E}+06$ & 0.1579 & 48.7 & 44.5 & 8.8 \\
\hline 21 & 12 & 3 & $1.804 \mathrm{E}+05$ & 150 & $9.019 \mathrm{E}+06$ & 0.0200 & 67.3 & 5.7 & 3.3 \\
\hline 22 & 16 & 81 & $3.653 \mathrm{E}+06$ & 102 & $4.600 \mathrm{E}+06$ & 0.7941 & 34.3 & 220.8 & 33.0 \\
\hline 23 & 20 & 2 & $7.215 \mathrm{E}+04$ & 78 & $2.814 \mathrm{E}+06$ & 0.0256 & 21.0 & 7.2 & 5.2 \\
\hline 24 & 16 & 5 & $2.255 \mathrm{E}+05$ & 173 & $7.801 \mathrm{E}+06$ & 0.0289 & 58.3 & 8.2 & 3.7 \\
\hline 25 & 60 & 62 & $7.456 \mathrm{E}+05$ & 615 & $7.395 \mathrm{E}+06$ & 0.1008 & 55.2 & 28.5 & 3.8 \\
\hline 26 & 14 & 15 & $7.730 \mathrm{E}+05$ & 25 & $1.288 \mathrm{E}+06$ & 0.6000 & 9.6 & 167.5 & 54.8 \\
\hline 27 & 25 & 5 & $1.443 E+05$ & 125 & $3.608 \mathrm{E}+06$ & 0.0400 & 26.9 & 11.3 & 5.2 \\
\hline 28 & 50 & 1 & $1.443 E+04$ & 130 & $1.876 \mathrm{E}+06$ & 0.0077 & 14.0 & 2.2 & 2.2 \\
\hline 29 & 40 & 96 & $1.732 \mathrm{E}+06$ & 162 & $2.922 \mathrm{E}+06$ & 0.5926 & 21.8 & 165.5 & 21.5 \\
\hline 30 & 30 & 1 & $2.405 E+04$ & 77 & $1.852 \mathrm{E}+06$ & 0.0130 & 13.8 & 3.7 & 3.7 \\
\hline 31 & 20 & 3 & $1.082 \mathrm{E}+05$ & 218 & $7.864 \mathrm{E}+06$ & 0.0138 & 58.7 & 3.9 & 2.3 \\
\hline 32 & 12 & 16 & $9.620 \mathrm{E}+05$ & 268 & $1.611 \mathrm{E}+07$ & 0.0597 & 120.3 & 16.9 & 4.3 \\
\hline
\end{tabular}

Clift et al. 


$\begin{array}{lccccccccc}\text { Crystal } & \mathbf{N g} & \mathbf{N s} & \mathbf{r h o - s} & \mathbf{N i} & \mathbf{r h o}-\mathbf{i} & \mathbf{N s} / \mathbf{N i} & \begin{array}{c}\text { U } \\ (\mathbf{p p m})\end{array} & \begin{array}{c}\text { Age } \\ (\mathbf{M a})\end{array} & \begin{array}{c}\text { Error } \\ 33\end{array} \\ 15 & 1 & 4.810 \mathrm{E}+04 & 21 & 1.010 \mathrm{E}+06 & 0.0476 & 7.5 & 13.5 & 13.8 \\ 34 & 15 & 33 & 1.587 \mathrm{E}+06 & 41 & 1.972 \mathrm{E}+06 & 0.8049 & 14.7 & 223.8 & 52.4 \\ 35 & 15 & 11 & 5.291 \mathrm{E}+05 & 132 & 6.349 \mathrm{E}+06 & 0.0833 & 47.4 & 23.5 & 7.4 \\ 36 & 14 & 16 & 8.246 \mathrm{E}+05 & 23 & 1.185 \mathrm{E}+06 & 0.6957 & 8.9 & 193.9 & 63.2 \\ 37 & 9 & 6 & 4.810 \mathrm{E}+05 & 7 & 5.612 \mathrm{E}+05 & 0.8571 & 4.2 & 238.0 & 132.5 \\ 38 & 12 & 6 & 3.608 \mathrm{E}+05 & 56 & 3.367 \mathrm{E}+06 & 0.1071 & 25.1 & 30.2 & 13.0 \\ 39 & 12 & 22 & 1.323 \mathrm{E}+06 & 54 & 3.247 \mathrm{E}+06 & 0.4074 & 24.2 & 114.2 & 28.9 \\ 40 & 8 & 18 & 1.623 \mathrm{E}+06 & 32 & 2.886 \mathrm{E}+06 & 0.5625 & 21.6 & 157.2 & 46.4 \\ 41 & 18 & 19 & 7.616 \mathrm{E}+05 & 33 & 1.323 \mathrm{E}+06 & 0.5758 & 9.9 & 160.9 & 46.4 \\ 42 & 12 & 6 & 3.608 \mathrm{E}+05 & 163 & 9.800 \mathrm{E}+06 & 0.0368 & 73.2 & 10.4 & 4.3 \\ 43 & 50 & 2 & 2.886 \mathrm{E}+04 & 136 & 1.962 \mathrm{E}+06 & 0.0147 & 14.7 & 4.2 & 3.0 \\ 44 & 25 & 5 & 1.443 \mathrm{E}+05 & 142 & 4.098 \mathrm{E}+06 & 0.0352 & 30.6 & 10.0 & 4.5 \\ 45 & 30 & 1 & 2.405 \mathrm{E}+04 & 96 & 2.309 \mathrm{E}+06 & 0.0104 & 17.2 & 2.9 & 3.0 \\ 46 & 15 & 7 & 3.367 \mathrm{E}+05 & 117 & 5.628 \mathrm{E}+06 & 0.0598 & 42.0 & 16.9 & 6.6 \\ 47 & 40 & 4 & 7.215 \mathrm{E}+04 & 164 & 2.958 \mathrm{E}+06 & 0.0244 & 22.1 & 6.9 & 3.5 \\ 48 & 20 & 2 & 7.215 \mathrm{E}+04 & 94 & 3.391 \mathrm{E}+06 & 0.0213 & 25.3 & 6.0 & 4.3 \\ 49 & 12 & 7 & 4.209 \mathrm{E}+05 & 15 & 9.019 \mathrm{E}+05 & 0.4667 & 6.7 & 130.7 & 59.9 \\ 50 & 24 & 6 & 1.804 \mathrm{E}+05 & 218 & 6.554 \mathrm{E}+06 & 0.0275 & 48.9 & 7.8 & 3.2\end{array}$

Clift et al. 